Reprinted from:

Algebraic Transformation Groups and Algebraic Varieties, Encyclopaedia of Mathematical Sciences, Vol. 132,

Subseries Invariant Theory and Algebraic Transformation Groups, Vol. III, Springer-Verlag, 2004

\title{
REPRESENTATION THEORY AND PROJECTIVE GEOMETRY
}

\author{
Joseph M. LAndsberg, Laurent Manivel
}




\title{
Representation Theory and Projective Geometry
}

\author{
Joseph M. Landsberg ${ }^{1}$ and Laurent Manivel $^{2}$ \\ 1 Georgia Institute of Technology, School of Mathematics, Atlanta, GA \\ 30332-0160, USA. jml@math.gatech.edu \\ 2 Université Grenoble I, BP 74, Institut Fourier, UMR 5582 du CNRS, 38402 \\ Saint Martin d'Hères cedex, France. Laurent.Manivel@ujf-grenoble.fr
}

\begin{abstract}
This article consists of three parts that are largely independent of one another. The first part deals with the projective geometry of homogeneous varieties, in particular their secant and tangential varieties. It culminates with an elementary construction of the compact Hermitian symmetric spaces and the closed orbits in the projectivization of the adjoint representation of a simple Lie algebra. The second part discusses division algebras, triality, Jordan agebras and the Freudenthal magic square. The third part describes work of Deligne and Vogel inspired by knot theory and several perspectives for understanding this work.
\end{abstract}

\section{Overview}

This article has two purposes. The first is to provide an elementary introduction to papers $[59,60,61,62,63]$, related works and their historical context. Sections 2.1-2.3, 2.5-2.6, 3.1-3.5, 4.1-4.3 should be accessible to a general audience of mathematicians. The second is to provide generalizations, new perspectives, and complements to results in these papers, i.e., things we thought of after the papers were published. In particular, we mention 2.5, 2.7, 3.4 and 3.6-3.9. Each section begins with a description of its contents.

Simply put, our goals are to use geometry to solve questions in representation theory and to use representation theory to solve questions in geometry.

On the geometric side, the objects of interest are homogeneous varieties $X=G / P \subset \mathbb{P} V$. Here $G$ is a complex semi-simple Lie group, $V$ is an irreducible $G$-module, $X$ is the unique closed orbit (projectivization of the orbit of a highest weight vector) and $P$ is the stabilizer of a point. For example, let $W=\mathbb{C}^{m}$ and let $X=G(k, W) \subset \mathbb{P}\left(\Lambda^{k} W\right)$, the Grassmannian of $k$-planes through the origin in $W$. Here $G / P=\operatorname{SL}(W) / P, V=\Lambda^{k} W$. We are more generally interested in the geometry of orbit closures in $\mathbb{P} V$.

Basic questions one can ask about a variety are its dimension, its degree, and more generally its Hilbert function. We may ask the same questions for 
varieties associated to $X$. For example the degrees of the dual varieties of Grassmannians are still unknown in general (see [67, 26, 78]).

Other types of problems include recognition questions. For example, given a variety with certain geometric properties, are those properties enough to characterize the variety? For an example of this, see Zak's theorem on Severi varieties below in Section 2.6. For another example, Hwang and Mok characterize rational homogeneous varieties by the variety of tangent directions to minimal degree rational curves passing through a general point, see [44] for an overview. Some results along those lines are described in Section 2.5 below. We also mention the LeBrun-Salamon conjecture [68, 69] which states that any Fano variety equipped with a holomorphic contact structure must be the closed orbit in the adjoint representation $X_{\text {ad }} \subset \mathbb{P} \mathfrak{g}$ for a complex simple Lie algebra. In this context also see $[8,52]$.

On the representation theory side, the basic objects are $\mathfrak{g}$, a complex semi-simple Lie algebra and $V$, an irreducible $\mathfrak{g}$-module (e.g., $\mathfrak{g}=\mathfrak{s t}(W)$, $\left.V=\Lambda^{k} W\right)$. Problems include the classification of orbit closures in $\mathbb{P} V$, to construct explicit models for the group action, to geometrically interpret the decomposition of $V^{\otimes k}$ into irreducible $\mathfrak{g}$-modules. We discuss these classical questions below, primarily for algebras occuring in "series".

Vassiliev theory points to the need for defining objects more general than Lie algebras. We have nothing to add about this subject at the moment, but the results of $[62,63]$ were partly inspired by work of Deligne [24] and Vogel $[82,83]$ in this direction.

For the mystically inclined, there are many strange formulas related to the exceptional groups. We present some such formulas in Sections 4.3, 4.6 below. Proctor and Gelfand-Zelevinski filled in "holes" in the classical formulas for the $\mathfrak{o s p}_{n}$ series using the non-reductive odd symplectic groups. Our formulas led us to exceptional analogues of the odd symplectic groups. These analogues are currently under investigation (see [65]).

When not otherwise specified, we use the ordering of roots as in [9].

We now turn to details. We begin with some observations that lead to interesting rational maps of projective spaces.

\section{Construction Of Complex Simple Lie Algebras Via Geometry}

We begin in Section 2.1 with three ingredients that go into our study: local differential geometry (asymptotic directions), elementary algebraic geometry (rational maps of projective space) and homogeneous varieties (the correspondence between rational homogeneous varieties and marked Dynkin diagrams). We then, in Sections 2.2-2.4 describe two algorithms that construct new varieties from old that lead to new proofs of the classification of compact Hermitian symmetric spaces and the Cartan-Killing classification of complex simple Lie algebras. The proofs are constructive, via explicit rational maps and in 
Section 2.5 we describe applications and generalizations of these maps. Our maps generalize maps used by Zak in his classification of Severi varieties and in Section 2.6 we describe his influence on our work. In Section 2.7 we return to a topic raised in Section 2.1, where we determined the parameter space of lines through a point of a homogeneous variety $X=G / P$. We explain Tits' correspondences which allow one to determine the parameter space of all lines on $X$ and in fact parameter spaces for all $G$-homogeneous varieties on $X$. We explain how to use Tits correspondences to explicitly construct certain homogeneous vector bundles, an in turn to use the explicit construction to systematize Kempf's method for desingularizing orbit closures.

\subsection{Differential Geometry, Algebraic Geometry and Representation Theory}

\subsubsection{Local Differential Geometry}

Let $X^{n} \subset \mathbb{P}^{n+a}$ be an algebraic variety.

Question 2.1. How to study the geometry of $X$ ?

One answer: Take out a microscope. Fix a smooth point $x \in X$ and take derivatives at $x$. The first derivatives don't yield much. One gets $\tilde{T}_{x} X$, the embedded tangent projective space, the union of lines (linearly embedded $\mathbb{P}^{1}$ 's) having contact to order one with $X$ at $x$. (Contact to order zero means the line passes through $x$.)

Sometimes we work with vector spaces so for $Y \subset \mathbb{P} V$ we let $\hat{Y} \subset V$ denote the cone over $Y$ and we let $\hat{T}_{x} X=\hat{\tilde{T}}_{x} X$. Let $T_{x} X$ denote the Zariski (intrinsic) tangent space to $X$ at $x$. We have $T_{x} X=\hat{x}^{*} \otimes \hat{T}_{x} X / \hat{x}$, see [59].

Taking second derivatives one obtains a variety $Y=Y_{X, x} \subset \mathbb{P} T_{x} X$ whose points are the asymptotic directions, the tangent directions to lines having contact to order two with $X$ at $x$.
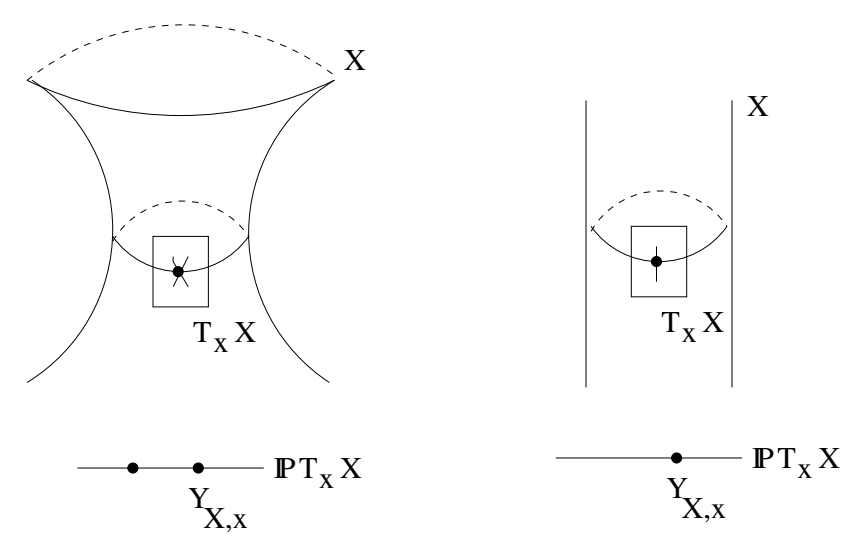
In an attempt to get global information from the asymptotic directions, restrict to the case where $x \in X$ is a general point

Question 2.2. How much of the geometry of $X$ can be recovered from $Y_{X, x} \subset$ $\mathbb{P} T_{x} X$ ?

Answer: Usually not much. For example, if $X$ is a smooth hypersurface, then $Y$ is always a smooth quadric hypersurface, i.e., all smooth hypersurfaces look the same to second order.

The set of asymptotic directions is the zero set of a system of quadratic equations generically of dimension equal to the codimension of $X$ (unless the codimension of $X$ is large, in which case it is generically the complete system of quadrics). If $Y$ is sufficiently pathological one might hope to recover important information about $X$.

Consider the Segre variety, $\operatorname{Seg}\left(\mathbb{P}^{k} \times \mathbb{P}^{l}\right) \subset \mathbb{P}\left(\mathbb{C}^{k+1} \otimes \mathbb{C}^{l+1}\right)$ of rank one matrices in the space of all $(k+1) \times(l+1)$ matrices. A short calculation shows $Y_{\mathrm{Seg}, x}=\mathbb{P}^{k-1} \sqcup \mathbb{P}^{l-1}$, the disjoint union of a $\mathbb{P}^{k-1}$ with a $\mathbb{P}^{l-1}$. (Note that the codimension is sufficiently large here that one would expect $Y$ to be empty based on dimension considerations.)

Griffiths and Harris [37] conjectured that if $Z \subset \mathbb{P}^{8}$ is a variety of dimension 4 and $z \in Z$ a general point, if $Y_{Z, z} \subset \mathbb{P} T_{z} Z$ is the disjoint union of two lines, then $Z=\operatorname{Seg}\left(\mathbb{P}^{2} \times \mathbb{P}^{2}\right) \subset \mathbb{P}^{8}$.

Theorem 2.3. [58] Let $k, l>1$, let $Z^{k+l} \subset \mathbb{P}^{N}$ be a variety, and let $z \in Z$ be a general point. If $Y_{Z, z}=\mathbb{P}^{k-1} \sqcup \mathbb{P}^{l-1}$, then $Z=\operatorname{Seg}\left(\mathbb{P}^{k} \times \mathbb{P}^{l}\right)$.

Moreover, the analogous rigidity is true for varieties having the same asymptotic directions as $X=G(2, m) \subset \mathbb{P}\left(\Lambda^{2} \mathbb{C}^{m}\right)$, the Grassmannian of two-planes in $\mathbb{C}^{m}$ in its Plucker embedding, with $m \geq 6$, and the complex Cayley plane $E_{6} / P_{6}=\mathbb{O P}_{\mathbb{C}}^{2} \subset \mathbb{P}^{26}[58]$. We recently prove similar rigidity results for homogeneous Legendrian varieties [64].

The rigidity results hold in the $C^{\infty}$ category if one replaces the word "general" by the word "every".

If one adds the global assumption that $Z$ is smooth, the results also hold for $X=G(2,5)$ and $X=\mathbb{S}_{5} \subset \mathbb{P}^{15}$, the spinor variety using different methods.

We are unaware of any second order rigidity results for nonhomogeneous varieties. Thus, purely from the most naive differential geometry, one already encounters homogeneous varieties as examples of the most rigid projective varieties. In fact, so far we have just encountered the most homogeneous ones, the ones admitting Hermitian symmetric metrics. For more on this and the rigidity of other homogenous varieties see Section 2.5.

We define a homogeneous variety $X=G / P \subset \mathbb{P} V$ to be minuscule if $G$ is simple, $X$ admits a Hermitian symmetric metric and $X$ is in its minimal homogeneous embedding. $X$ is said to be generalized minuscule if it is homogeneously embedded and admits a Hermitian symmetric metric. 
Remark 2.4. If $X \subset \mathbb{P} V$ is a variety cut out by quadratic polynomials, then the asymptotic directions $Y \subset \mathbb{P} T_{x} X$ are actually the tangent directions to lines (linearly embedded $\mathbb{P}^{1}$ 's) on $X$. Rational homogeneous varieties are cut out by quadratic equations, in fact if $X \subset \mathbb{P} V_{\lambda}$, then the ideal is generated by $V_{2 \lambda}^{\perp} \subset S^{2} V_{\lambda}^{*}$.

\subsubsection{Lie Groups and Homogeneous Varieties}

Let $G$ be a complex (semi)-simple Lie group and let $V$ be an irreducible $G$-module. Then there exists a unique closed orbit $X=G / P \subset \mathbb{P} V$.

Examples. 1. $G=\mathrm{SL}(n, \mathbb{C})$, the group preserving $\operatorname{det} \in \Lambda^{n} \mathbb{C}^{n *}, V=\Lambda^{k} \mathbb{C}^{n}$, $X=G(k, n)$, the Grassmannian of $k$-planes through the origin in $\mathbb{C}^{n}$.

2. $G=\mathrm{SO}(n, Q)$, the group preserving a nondegenerate $Q \in S^{2} \mathbb{C}^{n *}, V=$ $\Lambda^{k} \mathbb{C}^{n}, X=G_{Q}(k, n)$, the Grassmannian of $Q$-isotropic $k$-planes through the origin in $\mathbb{C}^{n}$.

3. $G=\operatorname{Sp}(n, \omega)$, the group preserving a nondegenerate $\omega \in \Lambda^{2} \mathbb{C}^{n *}, V=$ $\Lambda^{k} \mathbb{C}^{n} /\left(\Lambda^{k-2} \mathbb{C}^{n} \wedge \omega\right), X=G_{\omega}(k, n)$, the Grassmannian of $\omega$-isotropic $k$-planes through the origin in $\mathbb{C}^{n}$. Here $n$ is usually required to be even (but see $[73,36]$, and Section 4.3).

Since linear algebra is easier than global geometry, we work with $\mathfrak{g}=T_{\text {Id }} G$, the associated Lie algebra.

About a century ago, Killing and Cartan classified complex simple Lie algebras. Thanks to Coxeter and Dynkin, the classification can be expressed pictorially. (See [40] for a wonderful account of their work and the history surrounding it.)

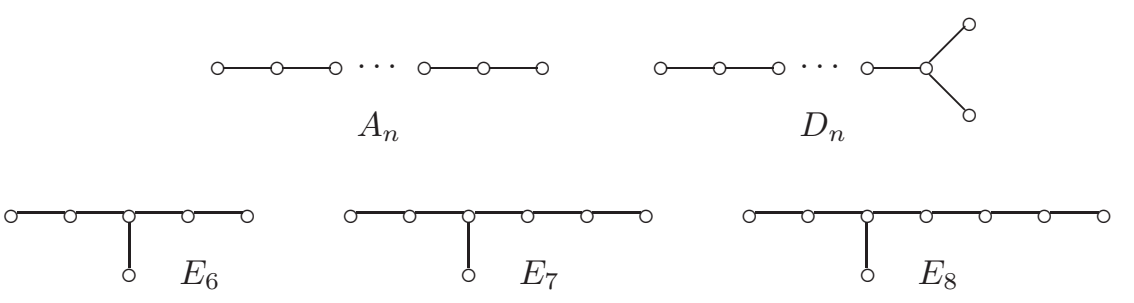

If a diagram has symmetry, we are allowed to fold it along the symmetry and place an arrow pointing away from the hinge to get a new one:

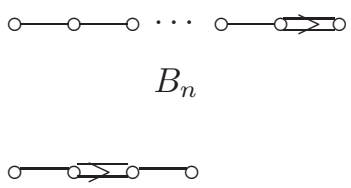

$F_{4}$

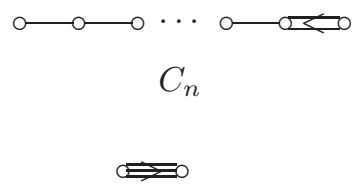

$G_{2}$ 
For example, $C_{n}$ is the fold of $A_{2 n-1}$. Given a semi-simple Lie algebra $\mathfrak{g}$, we let $D(\mathfrak{g})$ denote its Dynkin diagram.

Returning to geometry, homogeneous varieties correspond to marked diagrams
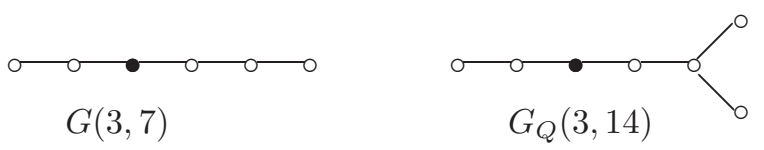

Note in particular that $\mathbb{P}^{n}$ has marked diagram

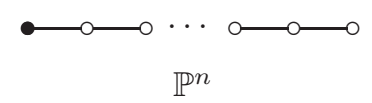

If $S$ is a subset of the simple roots of $G$, we use $P_{S}$ to designate the parabolic subgroup of $G$ corresponding to the simple roots in the complement of $S$. The Dynkin diagram for $G / P_{S}$ is the diagram for $G$ with roots in $S$ marked. Also, if $S$ consists of a single root $\{\alpha\}$ and $\alpha=\alpha_{i}$, we write $P_{S}=$ $P_{\alpha}=P_{i}$.

Unless otherwise specified, we take $\mathbb{P} V$ to be ambient space for the the minimal homogeneous embedding of $G / P$. For example, if $P=P_{\alpha}$ is maximal, then $V=V_{\omega}$ where $\omega$ is the weight Killing-dual to the coroot of $\alpha$.

Back to our question of how to study homogeneous varieties:

Idea: Study $X \subset \mathbb{P} V$ via $Y=Y_{X, x} \subset \mathbb{P} T_{x} X$.

For this idea to be a good one, $Y$ should be a simpler space than $X$ and it should be possible to determine $Y$ systematically. Although $Y$ is indeed cut out by quadratic equations, and has dimension strictly less than that of $X$, $X$ is homogeneous, and $Y$ need not be.

The following theorem originates with work of Tits [81], amplified by Cohen and Cooperstein [18]:

Theorem 2.5. [59] Let $X=G / P_{\alpha} \subset \mathbb{P V}$ be a rational homogeneous variety such that $\alpha$ is not short (i.e., no arrow in $D(\mathfrak{g})$ points towards $\alpha$ ).

Then $Y \subset \mathbb{P} T_{x} X$ is homogeneous, in fact generalized minuscule (as defined above).

Moreover, $Y$ can be determined pictorially: remove the node corresponding to $\alpha$ from $D(\mathfrak{g})$ and mark the nodes that were adjacent to $\alpha$. One obtains a semi-simple Lie algebra $\mathfrak{h}$ with marked diagram. The resulting homogeneous space $H / Q$ is $Y$.

Example 2.6.

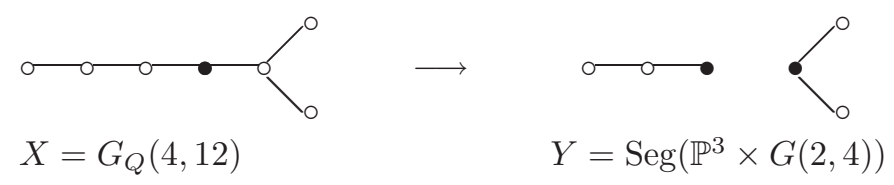


The $H$-modules $\langle Y\rangle$ are studied in [49] where they are called type- $I \theta$ representations.

The embedding of $Y$ is minimal iff the diagram is simply laced. In the case of a double edge, one takes the quadratic Veronese embedding, for a triple edge, one takes the cubic Veronese (see the algorithms below).

Remark 2.7. In [59] we explicitly determine $Y$ in the case of short roots and in fact arbitrary parabolics. We also give geometric models. In the case of $X=G / P_{\alpha}$ with $\alpha$ short, $Y$ is the union of exactly two $G$-orbits, and the closed orbit has codimension one in $Y$.

If $Y$ is still complicated, one can continue, studying the asymptotic directions of $Y$ at a point. Eventually one gets (Segre products of re-embeddings of) $\mathbb{C P}^{1}$, the one homogeneous space we all pretend to understand.

We describe our discovery that one can reverse this infinitesimalization procedure below. First we need to review some elementary algebraic geometry.

\subsubsection{Embeddings Of Projective Space}

Recall the Veronese embeddings of projective space:

$$
\begin{aligned}
v_{d}: \mathbb{P} V & \rightarrow \mathbb{P}\left(S^{d} V\right), \\
{[w] } & \mapsto\left[w^{d}\right] .
\end{aligned}
$$

Dually, let $P_{0}, \ldots, P_{N}$ be a basis of $S^{d} V^{*}$. The map is

$$
[w] \mapsto\left[P_{0}(w), \ldots, P_{N}(w)\right] .
$$

A remarkable fact is that all maps between projective spaces $\mathbb{P} V \rightarrow \mathbb{P} W$ are obtained by projecting a Veronese re-embedding.

If we project to $\mathbb{P}\left(S^{d} V / L\right)$ where $L^{\perp} \subset S^{d} V^{*}$ has basis $Q_{0}, \ldots, Q_{m}$, the map is

$$
\begin{aligned}
f: \mathbb{P} V & \rightarrow \mathbb{P}\left(S^{d} V / L\right), \\
{[w] } & \mapsto\left[Q_{0}(w), \ldots, Q_{m}(w)\right]
\end{aligned}
$$

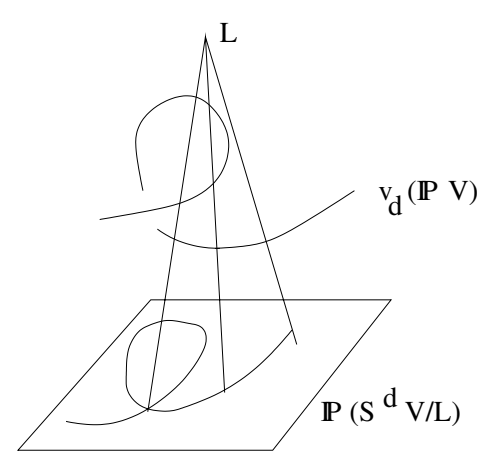


The image of $f$ is smooth and isomorphic to $\mathbb{P} V$ iff no secant line of $v_{d}(\mathbb{P} V)$ intersects $L$.

In the following algorithm we will actually be interested in images that get squashed in the projection so that they are no longer isomorphic to $\mathbb{P}^{n}$ (but are still smooth).

\subsection{First Algorithm}

We are about to describe an algorithm, which you might like to think of as a game. Starting with $\mathbb{P}^{1}$ as initial input we build some new algebraic varieties subject to certain rules. The game has rounds, and in each new round, we are allowed to use the outputs from previous rounds as new inputs.

We fix some notation.

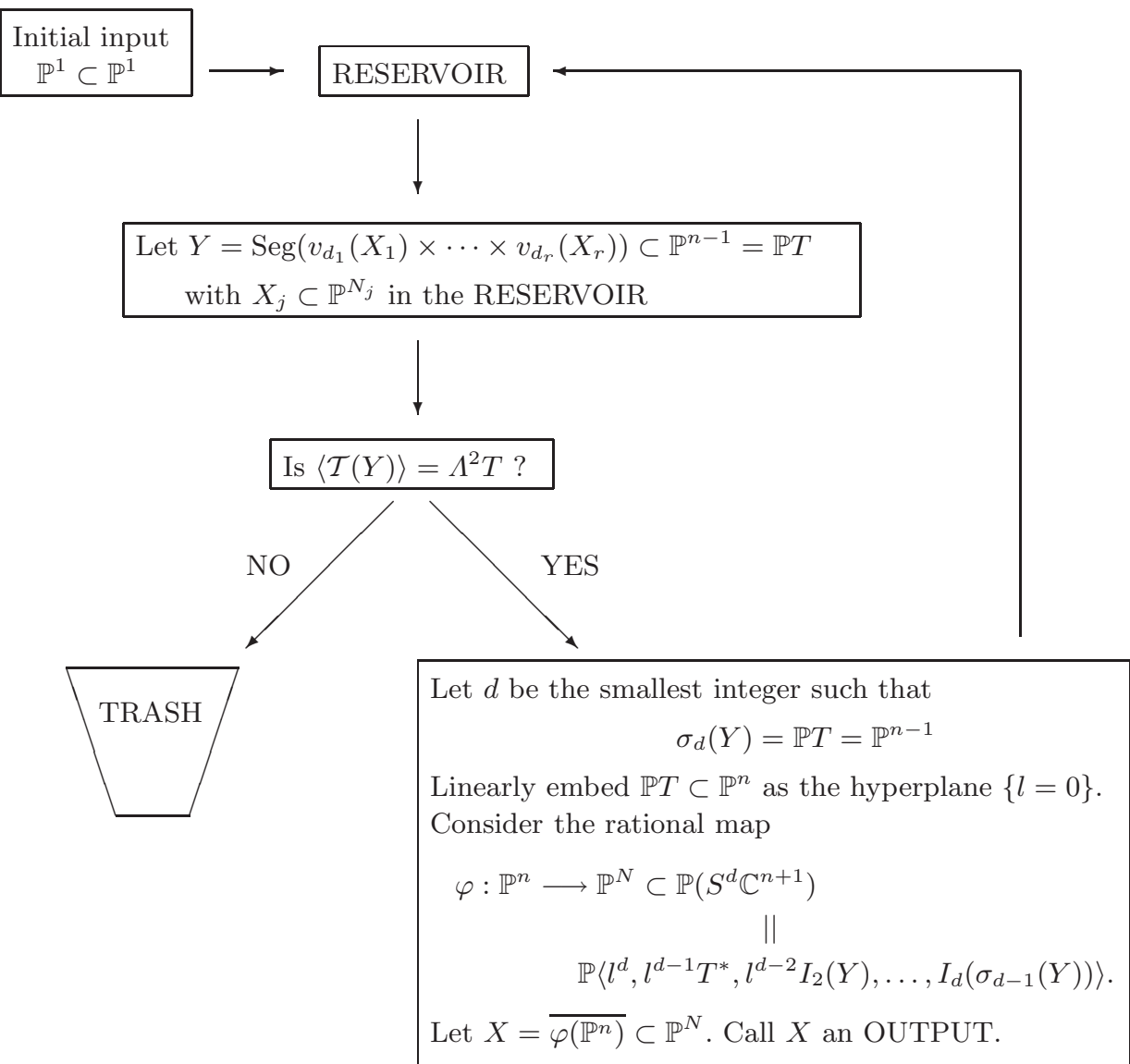

Fig. 1. First Algorithm 
Let $X \subset \mathbb{P} V, Y \subset \mathbb{P} W$, be varieties.

$\operatorname{Seg}(X \times Y) \subset \mathbb{P}(V \otimes W)$ is the Segre product, $([x],[y]) \mapsto[x \otimes y]$. Sometimes we just write $X \times Y$ with the Segre product being understood.

$\sigma_{k}(X) \subset \mathbb{P} V$ is the union of all secant $\mathbb{P}^{k-1}$ 's to $X$. We let $\sigma(X)=\sigma_{2}(X)$.

$\mathcal{T}(X) \subset G(2, V) \subset \mathbb{P}\left(\Lambda^{2} \mathbb{P} V\right)$ denotes the union of all tangent lines to $V$. (Recall $G(k, V)$ is also the space of $\mathbb{P}^{k-1}$ 's in $\mathbb{P} V$.)

$\hat{X} \subset V$ is the cone over $X$ and $\langle X\rangle \subseteq V$ denotes the linear span of $\hat{X}$.

Given $Y \subset \mathbb{P} W$, we let $I_{k}(Y) \subset \bar{S}^{k} W^{*}$ denote the component of the ideal in degree $k$. In the flowchart below we slightly abuse notation by letting $I_{k}(Y)$ denote a set of generators of $I_{k}(Y)$. Similarly, we let $T^{*}$ be shorthand for a basis of $T^{*}$.

For the first run through the algorithm, the admissible varieties and their outputs are as follows

$$
\begin{array}{rlrl}
Y & \subseteq \mathbb{P}^{n-1}, & X^{n} \subseteq \mathbb{P}^{N}, \\
\mathbb{P}^{1} & \subseteq \mathbb{P}^{1}, & \mathbb{P}^{2} & \subseteq \mathbb{P}^{2} \\
\mathbb{P}^{1} \times \mathbb{P}^{1} & \subset \mathbb{P}^{3}, & \mathbb{Q}^{4} & \subset \mathbb{P}^{5} \\
v_{2}\left(\mathbb{P}^{1}\right) & \subset \mathbb{P}^{2}, & \mathbb{Q}^{3} & \subset \mathbb{P}^{4} .
\end{array}
$$

Here and below, $\mathbb{Q}^{m} \subset \mathbb{P}^{m+1}$ denotes the smooth quadric hypersurface. For the second round,

$$
\begin{aligned}
Y & \subseteq \mathbb{P}^{n-1}, & X^{n} & \subseteq \mathbb{P}^{N}, \\
\mathbb{P}^{2} & \subseteq \mathbb{P}^{2}, & \mathbb{P}^{3} & \subseteq \mathbb{P}^{3}, \\
v_{2}\left(\mathbb{P}^{2}\right) & \subset \mathbb{P}^{5}, & G_{\omega}(3,6) & \subset \mathbb{P}^{11}, \\
\mathbb{P}^{1} \times \mathbb{P}^{2} & \subset \mathbb{P}^{5}, & G(2,5) & \subset \mathbb{P}^{9}, \\
\mathbb{P}^{2} \times \mathbb{P}^{2} & \subset \mathbb{P}^{8}, & G(3,6) & \subset \mathbb{P}^{19}, \\
\mathbb{Q}^{4} & \subset \mathbb{P}^{5}, & \mathbb{Q}^{6} & \subset \mathbb{P}^{8}, \\
\mathbb{Q}^{3} & \subset \mathbb{P}^{4}, & \mathbb{Q}^{5} & \subset \mathbb{P}^{7} .
\end{aligned}
$$

Here $G(k, l)$ denotes the Grassmannian of $k$-planes in $\mathbb{C}^{l}$ and $G_{\omega}(k, 2 k)$ denotes the Grassmannian of Lagrangian $k$-planes for a given symplectic form.

Question 2.8. What comes out? Since the algorithm goes on forever, is it even possible to answer this question?

Proposition 2.9. [60] The algorithm is effective. A priori, $r, d_{j} \leq 2$ and the algorithm stabilizes after six rounds.

So at least our question is reasonable. Now for the answer:

Theorem 2.10. [60] OUTPUTS = MINUSCULE VARIETIES.

Corollary 2.11. [60] A new proof of the classification of the compact Hermitian symmetric spaces without any reference to Lie groups. 
We have the following stable round:

$$
\begin{array}{rlrl}
Y & \subseteq \mathbb{P}^{n-1}, & X^{n} & \subseteq \mathbb{P}^{N}, \\
\mathbb{P}^{n-1} & \subseteq \mathbb{P}^{n-1}, & \subseteq \mathbb{P}^{n} \\
v_{2}\left(\mathbb{P}^{m-1}\right) & \subset \mathbb{P}^{\left(\begin{array}{c}
m+1 \\
2
\end{array}\right)-1}, & G_{\omega}(m, 2 m) & \subset \mathbb{P}^{C_{m+1}-1}, \\
\mathbb{P}^{k-1} \times \mathbb{P}^{l-1} & \subset \mathbb{P}^{k l-1}, & G(k, k+l) & \subset \mathbb{P}^{\left(\begin{array}{c}
k+l \\
k
\end{array}\right)-1} \\
\mathbb{Q}^{2 m-2} & \subset \mathbb{P}^{2 m-1}, & \mathbb{Q}^{2 m} & \subset \mathbb{P}^{2 m+1}, \\
\mathbb{Q}^{2 m-1} & \subset \mathbb{P}^{2 m}, & \mathbb{Q}^{2 m+1} & \subset \mathbb{P}^{2 m+2}, \\
G(2, m) & \subset \mathbb{P}^{\left(\begin{array}{c}
m \\
2
\end{array}\right)-1}, & \mathbb{S}_{m} & \subset \mathbb{P}^{2^{m-1}-1}
\end{array}
$$

Here $C_{m+1}=\frac{1}{m+2}\left(\begin{array}{c}2 m+2 \\ m+1\end{array}\right)$ is the $(m+1)$-st Catalan number. The spinor variety $\mathbb{S}_{m}$ of $D_{m}$ consists of one family of maximal isotropic subspaces of $\mathbb{C}^{2 m}$ endowed with a nondegenerate quadratic form and embedded in the projectivization of one of the two half-spin representations.

The most interesting (but terminal) path is:

$$
\begin{aligned}
\operatorname{Seg}\left(\mathbb{P}^{1} \times \mathbb{P}^{2}\right) & =A_{1} / P_{1} \times A_{2} / P_{2} \\
& \downarrow \\
G(2,5) & =A_{4} / P_{2} \\
& \downarrow \\
\mathbb{S}_{5} & =D_{5} / P_{5} \\
& \downarrow \\
\mathbb{O P}_{\mathbb{C}}^{2} & =E_{6} / P_{6} \\
& \downarrow \\
G_{w}\left(\mathbb{O}^{3}, \mathbb{O}^{6}\right) & =E_{7} / P_{7} .
\end{aligned}
$$

Here $\mathbb{O P}_{\mathbb{C}}^{2}=E_{6(-14)}$ is the complexification of $\mathbb{O P}^{2}=F_{4} / \operatorname{Spin}_{9}$, the celebrated Cayley plane discovered by Chevalley. As a topological space, $\mathbb{O P}^{2}$ is built out of three cells of dimension 0,8 and 16 . It is $F_{4(-20)}$ in the notation of Tits (see e.g. [70]). The notation $G_{w}\left(\mathbb{O}^{3}, \mathbb{O}^{6}\right)$ is discussed in Section 4.6.

\subsection{Second Algorithm}

Now that was fun, but it was a shame to throw away some of our favorite varieties like $v_{3}\left(\mathbb{P}^{1}\right)$ and $\operatorname{Seg}\left(\mathbb{P}^{1} \times \mathbb{P}^{1} \times \mathbb{P}^{1}\right)$. Let's revise the algorithm slightly and have just one consolation round. Given a smooth variety $Y \subset \mathbb{P} V$, we let $\tau(Y) \subset \mathbb{P} V$ denote the union of all points on all embedded tangent lines to $Y$.

This algorithm is also effective. We show a priori that $r, d_{j} \leq 3$.

So, what do we get???

Theorem 2.12. [60] OUTPUTS $=$ Fundamental adjoint varieties $X \subset \mathbb{P} \mathfrak{g}$.

The fundamental adjoint varieties are the closed orbits in the adjoint representation when it is fundamental. The Lie algebras whose adjoint varieties are not fundamental are the pathological $A_{n}$ and its fold (when foldable) $C_{m}$. 


\section{MINUSCULE VARIETIES}

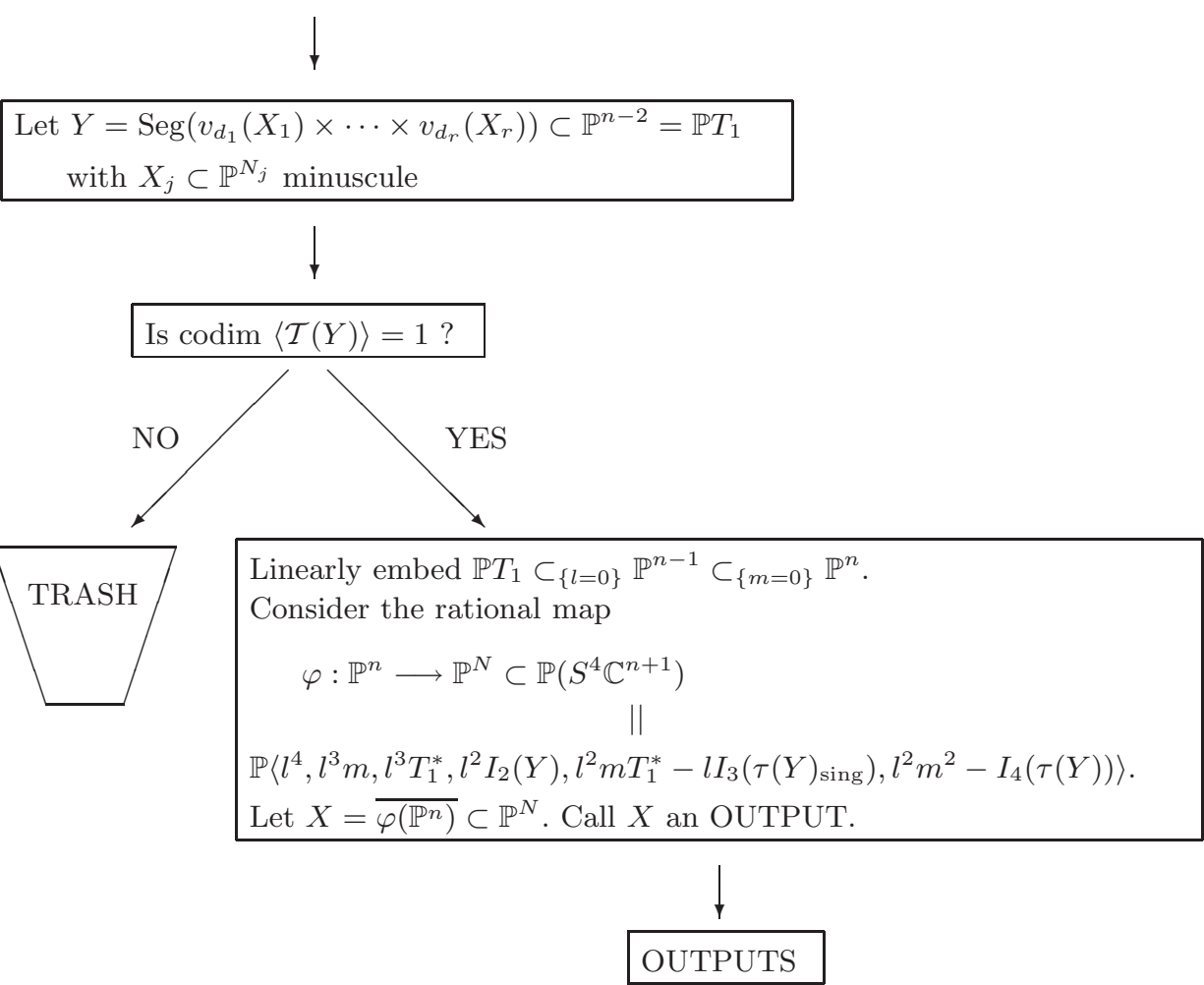

Fig. 2. Consolation prize (one round)

Our algorithm again provides a beautiful and easy proof of the classification of fundamental adjoint varieties. We also account for these two pathological cases, but it involves some less elegant work. We do obtain:

Corollary 2.13. [60] A new proof of the Cartan-Killing classification of complex simple Lie algebras.

The examples for the adjoint algorithm are as follows:

$$
\begin{array}{rlrl}
Y & \subset \mathbb{P}^{n-2}, & \mathfrak{g}, \\
v_{3}\left(\mathbb{P}^{1}\right) & \subset \mathbb{P}^{3}, & \mathfrak{g}_{2}, \\
\mathbb{P}^{1} \times \mathbb{Q}^{m-4} & \subset \mathbb{P}^{2 m-5}, \mathfrak{s o}_{m}, \\
G_{\omega}(3,6) & \subset \mathbb{P}^{13}, & \mathfrak{f}_{4}, \\
G(3,6) & \subset \mathbb{P}^{19}, & \mathfrak{e}_{6}, \\
\mathbb{S}_{6} & \subset \mathbb{P}^{31}, & \mathfrak{e}_{7}, \\
G_{w}\left(\mathbb{O}^{3}, \mathbb{O}^{6}\right) & \subset \mathbb{P}^{55}, & \mathfrak{e}_{8} .
\end{array}
$$


The two exceptional (i.e., non-fundamental) cases are

$$
\begin{aligned}
\mathbb{P}^{k-3} \sqcup \mathbb{P}^{k-3} & \subset \mathbb{P}^{2 k-3}, \quad \mathfrak{s l}_{k}, \\
\varnothing & \subset \mathbb{P}^{2 m-1}, \quad \mathfrak{s p}_{2 m} .
\end{aligned}
$$

Note that the algorithm works in these two cases, we just didn't have the varieties in our reservoir. (In the case of $\mathfrak{s p}_{2 m}$, it may have been there and just difficult to see.)

\subsection{Outline Of the Proofs}

The proofs have three ingredients: differential invariants, local Lie algebras, and relating Casimirs to geometry.

\subsubsection{Differential Invariants}

Given a variety $X \subset \mathbb{P} V$ and $x \in X$, we can recover $X$ from its Taylor series at $x$. The projective differential invariants comprise a series of tensors encoding the geometric (i.e., invariant under $G L(V)$ ) information in the Taylor series. We prove a priori facts about the differential invariants of any putative minuscule variety or fundamental adjoint variety.

\subsubsection{Local Lie Algebras}

A local Lie algebra is a graded vector space

$$
\mathfrak{g}_{-1} \oplus \mathfrak{g}_{0} \oplus \mathfrak{g}_{1}
$$

equipped with a bracket for which $\mathfrak{g}_{0}$ is a Lie algebra. The bracket must respect the grading but the Jacobi identity need not hold. If the Jacobi identity fails, one can construct a (unique) $\mathbb{Z}$-graded Lie algebra from the local Lie algebra. The traditional way to do this (see, e.g., [48]) is to take the free algebra generated by the brackets and then mod out by the relations. Note that if one does that, one has no idea how many factors (if any at all) one will be adding on to obtain the final result.

The data $Y=H / Q \subseteq \mathbb{P} T_{1}$ furnishes (up to scale) a local Lie algebra with $\mathfrak{g}_{0}=\mathfrak{h} \oplus \mathbb{C}, \mathfrak{g}_{1}=T_{1}, \mathfrak{g}_{-1}=T_{1}^{*}$. Here the action of $\mathbb{C}$ is as a scalar times the identity and we do not initially specify the scalar.

Since this does give rise to a unique $\mathbb{Z}$-graded Lie algebra, (in particular, a Lie algebra equipped with a representation $V$ supported on one fundamental weight), we can study the resulting homogeneous variety $X^{\prime}=G / P \subset \mathbb{P} V$, and calculate its differential invariants.

Note that if $X^{\prime}$ is minuscule, $\mathfrak{g}=\mathfrak{g}_{-1} \oplus \mathfrak{g}_{0} \oplus \mathfrak{g}_{1}$ and if $X^{\prime}$ is adjoint, $\mathfrak{g}=$ $\mathfrak{g}_{-2} \oplus \mathfrak{g}_{-1} \oplus \mathfrak{g}_{0} \oplus \mathfrak{g}_{1} \oplus \mathfrak{g}_{2}$ with $\mathfrak{g}_{ \pm 2}=\mathbb{C}$. 


\subsubsection{Compare $X^{\prime}$ With the Constructed Variety $X$}

We compare the differential invariants of $X$ and $X^{\prime}$ and show that they are the same. The key point in the minuscule case is that a local Lie algebra is already a Lie algebra iff the Jacobi identity holds. We show this is the case iff $\Lambda^{2} \mathfrak{g}_{1}$ is an eigenspace for the Casimir operator of $\mathfrak{h}$. We then connect this to the geometry by showing that $\langle\mathcal{T}(Y)\rangle$ is a Casimir eigenspace!

For the adjoint case the idea is similar: one shows that one can get away with a one-dimensional correction.

\subsection{Applications, Generalizations and Related Work}

\subsubsection{More General Algorithms}

The adjoint algorithm (with a different test for admission) also constructs all homogeneous varieties and representations corresponding to a five step grading. M. Dillon is currently formulating more general constructions for gradings including extensions to affine Lie algebras. (The algorithms have no hope of being effective for graded Lie algebras with exponential growth.) An amusing exercise is to construct the grading of $\mathfrak{e}_{8}{ }^{(1)}$ associated to $\alpha_{5}$. Here $\mathfrak{h}=\mathfrak{a}_{4}+\mathfrak{a}_{4}$, and

$$
\begin{gathered}
Y=Y_{1}=G(2,5) \times \mathbb{P}^{4}=\operatorname{Seg}(G(2, V) \times \mathbb{P} W) \subset \mathbb{P}\left(\Lambda^{2} V \otimes W\right)=\mathbb{P} \mathfrak{g}_{1}, \\
Y_{2}=\operatorname{Seg}\left(\mathbb{P} V^{*} \times G(2, W)\right) \subset \mathbb{P}\left(V^{*} \otimes \Lambda^{2} W\right)=\mathbb{P g}_{2}, \\
Y_{3}=\operatorname{Seg}\left(\mathbb{P} V \times G\left(2, W^{*}\right)\right) \subset \mathbb{P}\left(V \otimes \Lambda^{2} W^{*}\right)=\mathbb{P} \mathfrak{g}_{3}, \\
Y_{4}=\operatorname{Seg}\left(G(2, V) \times \mathbb{P} W^{*}\right) \subset \mathbb{P}\left(\Lambda^{2} V \otimes W^{*}\right)=\mathbb{P} \mathfrak{g}_{4},
\end{gathered}
$$

and then the cycle repeats. Note that all the varieties are isomorphic as varieties and they appear in all possible combinations in terms of the $\mathfrak{h}$-action.

In this context, a result of Kostant [56] (Theorem 1.50) is interesting. He determines, given a reductive Lie algebra $\mathfrak{r}$ and an $\mathfrak{r}$-module $V$, when $\mathfrak{r}+V$ can be given the structure of a Lie algebra compatible with the $\mathfrak{r}$-actions (and in how many different ways).

\subsubsection{Constructions In an Algebraic Context}

Let $\mathcal{A}$ be an algebra with unit and involution $a \mapsto \bar{a}$. B. Allison [2] defines $\mathcal{A}$ to be structurable if the following graded vector space is actually a graded Lie algebra. Let

$$
\mathfrak{g}(\mathcal{A})=\mathfrak{g}_{-2} \oplus \mathfrak{g}_{-1} \oplus \mathfrak{g}_{0} \oplus \mathfrak{g}_{1} \oplus \mathfrak{g}_{2}
$$

with $\mathfrak{g}_{ \pm 1} \simeq \mathcal{A}, \mathfrak{g}_{ \pm 2} \simeq \mathcal{S}(\mathcal{A}):=\{a \in \mathcal{A} \mid \bar{a}=-a\}$ and $\mathfrak{g}_{0}$ the set of derivations of $\mathcal{A}$ generated by linear maps $V_{a, b}: \mathcal{A} \rightarrow \mathcal{A}$, where $V_{a, b}(c)=(a \bar{b}) c+(c \bar{b}) a-$ $(c \bar{a}) b$.

$\mathfrak{g}(\mathcal{A})$ always has a natural bracket, and is a Lie algebra iff the Jacobi identity holds. In analogy with our situation, there is essentially one identity 
to check, which, in Allison's notation becomes

$$
\left[V_{a, 1}, V_{b, c}\right]=V_{V_{a, 1}(b), c}-V_{b, V_{\bar{a}, 1}(c)} .
$$

Allison shows there is a one to one correspondence between simple structurable algebras and certain symmetric five step gradings of simple Lie algebras. His constructions work over all fields and one motivation for his constructions was to determine simple Lie algebras over arbitrary fields. Our more general 5 -step constructions correspond to algebraic structures termed Kantor triple systems.

Jordan triple systems form a special class of Kantor triple systems, those with trivial involution $\bar{a}=a$ and thus $\mathcal{S}(\mathcal{A})=0$, and they give rise to the minuscule gradings our minuscule algorithm produces.

\subsubsection{Rigidity}

In contrast to the rigidity theorems in Section 2.1.1 we have:

Theorem 2.14. There exist "fake" adjoint varieties. That is, for each adjoint variety $X \subset \mathbb{P} \mathfrak{g}$, there exists $Z \subset \mathbb{P}^{N}$, not isomorphic to $X, U \subset Z$ a Zariski open subset, and a holomorphic map $\phi: U \rightarrow X$ such that asymptotic directions are preserved (in fact the entire projective second fundamental form is preserved, see [60]).

Moreover, the same is true for all non-minuscule homogeneous varieties.

This local flexibility fails globally for adjoint varieties. Consider the following result of Hong [42]:

Theorem 2.15. A Fano manifold with a geometric structure modeled after a fundamental adjoint variety $Z$ is biholomorphic to $Z$ and the geometric structure is locally isomorphic to the standard geometric structure on $Z$.

A geometric structure on a variety $X$ modeled after a fundamental adjoint variety $Z$ may be understood as follows. Tor all $x \in X$, one has a subvariety $Y_{x} \subset \mathbb{P} T_{x} X$ isomorphic to the asymptotic directions $Y_{z} \subset \mathbb{P} T_{z} Z$. Note that here the subvariety $Y_{x}$ is not required to play any particular role, but it must be present at every point so in particular it determines a family of distributions on $X$.

Hong's theorem is a variant of an earlier rigidity result of Hwang and Mok [45], where a geometric structure may be understood in the analogous way.

Theorem 2.16. A Fano manifold with a geometric structure modeled after a compact irreducible Hermitian symmetric space $S$ of rank $\geq 2$ is biholomorphic to $S$ and the geometric structure is locally isomorphic to the standard geometric structure on $S$. 
The work of Hwang and Mok (also see [44]) relies on studying an intrinsic analog of the set of asymptotic directions. Namely a Fano manifold $X$ is uniruled by rational curves, and, fixing a reference line bundle, one has a subvariety $Y_{x} \subset \mathbb{P} T_{x} X$ of tangent directions to minimal degree rational curves. They also study deformation rigidity of homogeneous varieties with Picard number one. It is worth remarking that in the case of Hermitian symmetric spaces [46], a key fact used in their proof is that $\mathcal{T}\left(Y_{x}\right) \subset \mathbb{P} T_{x} X$ is linearly nondegenerate, i.e., the same condition we use in the minuscule algorithm.

\subsubsection{Normal Forms For Singularities}

In [4], Arnold classified the simple singularities. Like many interesting things in life they are in correspondence with Dynkin diagrams, in fact just the simply laced ones. He also gave normal forms in a minimal set of variables (two). It was also known (but evidently unpublished) that the simple singularities can be realized as degree three hypersurface singularities if one allows the number of variables to grow with the Milnor number. Holweck [41] has found a nice realization of these hypersurface singularities using a theorem of Knop [54] and the construction in Section 2.3 .

\subsection{Why Secant and Tangent Lines?}

The idea that secant and tangent lines should so strongly control the geometry of homogeneous varieties was inspired by Zak's theorem on Severi varieties.

Theorem 2.17. (Zak's theorems on linear normality and Severi varieties) Let $X^{n} \subset \mathbb{P}^{n+a}$ be a smooth variety, not contained in a hyperplane and such that $\sigma(X) \neq \mathbb{P}^{n+a}$ Then

(i) $a \geq \frac{n}{2}+2$.

(ii) If $a=\frac{n}{2}+2$ then $X$ is one of $v_{2}\left(\mathbb{P}^{2}\right) \subset \mathbb{P}^{5}, \operatorname{Seg}\left(\mathbb{P}^{2} \times \mathbb{P}^{2}\right) \subset \mathbb{P}^{8}$, $G(2,6) \subset \mathbb{P}^{14}, \mathbb{O P}_{\mathbb{C}}^{2} \subset \mathbb{P}^{26}$.

The four critical varieties are called the Severi varieties after Severi who proved the $n=2$ case of the theorem.

It is not known if there is a bound on the secant defect $\delta_{\sigma}(X):=$ $2 n+1-\operatorname{dim} \sigma(\mathrm{X})$ for smooth subvarieties of projective space with degenerate secant varieties. On the other hand, Zak established an upper bound on the codimension of a smooth variety of a given secant defect. He then went on to classify the varieties achieving this bound, which he calls the Scorza varieties. They are all closed orbits $G / P \subset \mathbb{P} V$, namely $v_{2}\left(\mathbb{P}^{n}\right), \operatorname{Seg}\left(\mathbb{P}^{n} \times \mathbb{P}^{n}\right)$, $G(2, n)$ and $\mathbb{O} \mathbb{P}_{\mathbb{C}}^{2}$.

Using Zak's result, Ein and Shepherd-Baron proved these four varieties also classify quadro-quadro Cremona transformations [29]. There are numerous other characterization problems where the answer is the Severi varieties.

Zak's proofs of his theorems rely on looking at entry loci. Namely let $X \subset \mathbb{P} V$ be a variety and let $y \in \mathbb{P} V \backslash X$. Define the entry locus of $y$ to be 


$$
\Sigma_{y}:=\overline{\left\{x \in X \mid \exists z \in X, y \in \mathbb{P}_{x z}^{1}\right\}} .
$$

Here $\mathbb{P}_{x z}^{1}$ denotes the projective line spanned by $x$ and $z$. Zak shows that for a Severi variety, the entry locus of a general point of $\sigma(X)$ is a quadric hypersurface in $X$. He then goes on to show that a variety so uniruled by quadric hypersurfaces and satisfying the dimension requirements must satisfy further dimension restrictions and eventually must be one of the four Severi varieties. More precisely, he shows that each Severi variety is the image of a rational map of a projective space. Our algorithms generalize his construction of the Severi varieties.

Recently Chaput [15] has shown that such a uniruling by quadrics immediately implies homogeneity, which gives a quicker proof of Zak's theorem.

The rigidity results for the Severi varieties other than $v_{2}\left(\mathbb{P}^{2}\right)$, are an outgrowth of a different proof of Zak's theorem, where one first shows that any putative Severi variety infinitesimally looks like an actual Severi variety to second order at a general point, and then uses the rigidity to finish the proof. $\left(v_{2}\left(\mathbb{P}^{2}\right)\right.$ requires special treatment as its tangential variety is nondegenerate.) See $[57]$ and $[58]$.

Many other important problems are related to the geometry of secant varieties. Consider the classical Waring problem: given a generic homogeneous polynomial of degree $d$ in $n+1$ variables, what is the minimal $k$ such that the polynomial may be written as a sum of $k d$-th powers? Phrased geometrically, the problem is to find the minimal $k$ such that $\sigma_{k}\left(v_{d}\left(\mathbb{P}^{n}\right)\right)=\mathbb{P}\left(S^{d} \mathbb{C}^{n+1}\right)$. This problem was solved by Alexander and Hirschowitz [1]. Generalizations and variants are still open, see $[13,47,75]$ for recent progress.

\subsection{Tits Correspondences and Applications}

In Section 2.7.1 we describe a construction, Tits correspondences [81], to determine the homogeneous (in the sense described below) unirulings of a rational homogeneous variety $X \subset \mathbb{P} V$. We explain how to use Tits correspondences to explicitly construct the homogeneous vector bundles over $X$ in Section 2.7.2. In Section 2.7.3 we relate Tits correspondences to other orbit closures in $\mathbb{P} V$ and systematize Kempf's method for desingularizing orbit closures. Tits correspondences led us to the decomposition formulas described in Section 4.2 and we believe they will have more applications in the future.

\subsubsection{Tits Correspondences}

Tits associated to any simple group a full set of geometries, encoded by the parabolic subgroups and their relative positions. This culminated in the definition of buildings, which has since known formidable developments. The Tits correspondences we now describe come from this perspective.

Let $G$ be a simple Lie group, let $S, S^{\prime}$ be two subsets of simple roots of $G$. Consider the diagram 


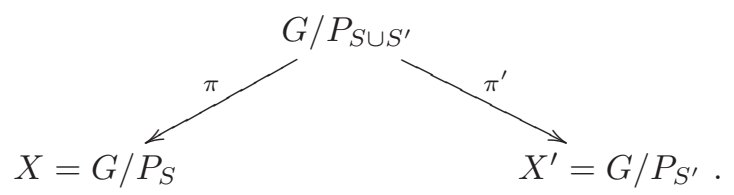

Let $x^{\prime} \in X^{\prime}$ and consider $Y:=\pi\left(\pi^{\prime-1}\left(x^{\prime}\right)\right) \subset X$. We call $Y=Y_{x^{\prime}}$ the Tits transform of $x^{\prime}$. The variety $X$ is covered by such varieties $Y$. Tits shows that $Y=H / Q$ where $\mathcal{D}(H)=\mathcal{D}(G) \backslash\left(S \backslash S^{\prime}\right)$, and $Q \subset H$ is the parabolic subgroup corresponding to $S^{\prime} \backslash S$. We call such subvarieties $Y$ of $X, G$-homogeneous subvarieties.

\subsubsection{Unirulings}

Unirulings by lines. Let $X=G / P_{\alpha} \subset \mathbb{P} V$ be a homogeneous variety. In Theorem 2.5 above we determined the parameter space of lines through a point of $X$. Here we determine the space of all lines on $X$. Let $\mathbb{F}_{1}(X)^{G} \subset G(2, V) \subset$ $\mathbb{P} \Lambda^{2} V$ denote the variety parametrizing the $G$-homogeneous $\mathbb{P}^{1}$ 's on $X$. Then $\mathbb{F}_{1}(X)^{G}=G / P_{S}$ where $S$ is the set of simple roots adjacent to $\alpha$ in $D(\mathfrak{g})$. If $P$ is not maximal, there is one family of $G$-homogeneous $\mathbb{P}^{1}$ 's for each marked node. If $\alpha$ is not a short root, all lines are $G$-homogeneous.

Unirulings by $\mathbb{P}^{k}$ 's. The diagram for $\mathbb{P}^{k}$ is $\left(\mathfrak{a}_{k}, \omega_{1}\right)$. Here there may be several $G$-homogeneous families of $\mathbb{P}^{k}$ 's on $X=G / P_{\alpha}$, each arising from a subdiagram of $D(\mathfrak{g}, \omega)$ isomorphic to a $D\left(\mathfrak{a}_{k}, \omega_{1}\right)$. If $\alpha$ is not short, then all $\mathbb{P}^{k}$ 's on $X$ are $G$-homogeneous.

Example 2.18. The largest linear space on $E_{n} / P_{1}$ is a $\mathbb{P}^{n-1}$, via the chain terminating with $\alpha_{n}$, so $E_{n} / P_{1}$ is maximally uniruled by $\mathbb{P}^{n-1}$ 's. There is a second chain terminating with $\alpha_{2}$, so $E_{n} / P_{1}$ is also maximally uniruled by $\mathbb{P}^{4}$ 's. (The unirulings by the $\mathbb{P}^{4}$ 's are maximal in the sense that none of the $\mathbb{P}^{4}$ 's of the uniruling are contained in any $\mathbb{P}^{5}$ on $E_{n} / P_{1}$.) The varieties parametrizing these two unirulings of $E_{n} / P_{1}$ are respectively $E_{n} / P_{2}$ and $E_{n} / P_{5}$.
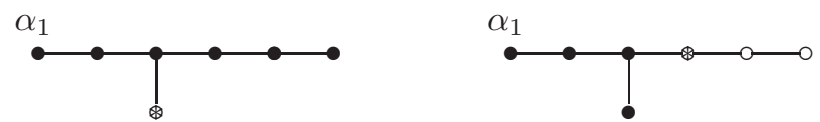

Unirulings by quadrics. Here we look for subdiagrams isomorphic to the diagram of a quadric hypersurface, i.e., $D\left(\mathfrak{s o}_{n}, \omega_{1}\right)$. Note that there are at most two possible such subdiagrams except for the case of $(\mathfrak{g}, V)=\left(\mathfrak{s o}_{8}, V_{\omega_{2}}\right)$ where there are three isomorphic ones.

Example 2.19. $(\mathfrak{g}, V)=\left(\mathfrak{s o}_{2 m}, V_{\omega_{k}}\right)$ has two such unirulings, by $\mathbb{Q}^{4}$ 's parametrized by $D_{m} / P_{\alpha_{k-2}, \alpha_{k+2}}$ and by $\mathbb{Q}^{2(m-k-1)}$ 's parametrized by $D_{m} / P_{\alpha_{k-1}}$. 

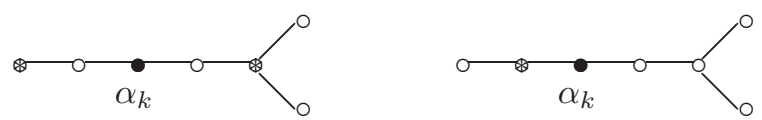

Example 2.20. (The Severi varieties) The $G$-homogeneous quadrics uniruling the Severi varieties are parametrized by the same Severi variety in the dual projective space.

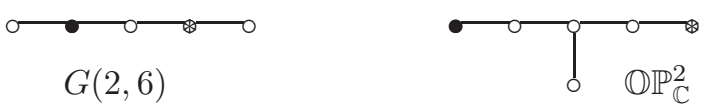

Unirulings by quadrics give rise to subvarieties of $\sigma(X)$ as follows. The union of the spans of the quadrics produce a subvariety of $\sigma(X)$ whose entry loci contain the quadrics (and in all examples we know, are equal to the quadrics). It also appears that, when $X$ is homogeneous, these give the subvarieties of $\sigma(X)$ with maximal entry loci. As explained below, these orbit closures admit uniform desingularizations by Kempf's method.

\subsubsection{Homogeneous Vector Bundles}

The $G$-homogeneous vector bundles over rational homogeneous varieties $Z=$ $G / Q$ are defined by $Q$-modules. (We change notation to reserve $G / P$ for a different role below.) If $W$ is a $Q$-module, one obtains the vector bundle $E_{W}:=G \times{ }_{Q} W \rightarrow G / Q$ where $(g p, w) \simeq(g, p w)$ for all $p \in Q$. Note that $D(\mathfrak{f})$ is $D(\mathfrak{g})$ with the nodes corresponding to $Q$ deleted.

Some homogeneous vector bundles over rational homogeneous varieties $Z=G / Q$ can be understood in terms of Tits fibrations. Let $X=G / P \subset \mathbb{P} V$. For each $z \in Z$ we obtain a variety $Y_{z} \subset X$ and thus a linear space $\left\langle Y_{z}\right\rangle \subset V$. As we vary $z$, we obtain a vector bundle $E \rightarrow Z$ whose fibers are the $\left\langle Y_{z}\right\rangle$ 's. In particular, $E$ is a subbundle of the trivial bundle $V \otimes \mathcal{O}_{Z} \rightarrow Z$.

\subsubsection{Collapses à la Kempf}

Let $X=G / P \subset \mathbb{P} V$ be a rational homogeneous variety. In [53], Kempf defined a method for desingularizing orbit closures $\overline{\mathcal{O}} \subset \mathbb{P} V$ by finding a vector bundle over a different homogeneous space $Z=G / Q$. He calls this technique the collapsing of a vector bundle. He gave some examples that appear to be found ad hoc. In [61] we gave new examples of collapsing occurring in series. Using the above discussion, we now give another description of Kempf's method.

\section{Recipe For Kempf's Method}

Let $V$ be a $G$-module with closed orbit $X \subset \mathbb{P} V$. Let $Z=G / Q$ be a rational homogeneous variety of $G$, defining a Tits correspondence with $X$. 
Consider the $G$-variety, which often appears to be an orbit closure

$$
\overline{\mathcal{O}}=\cup_{z \in Z}\left\langle Y_{z}\right\rangle \subset \mathbb{P} V .
$$

If $\overline{\mathcal{O}} \neq \mathbb{P} V$, then $\overline{\mathcal{O}}$ is a singular variety. It admits a desingularization by $\mathbb{P} E$, where $E \rightarrow Z$ is the vector bundle constructed above as the natural map $\mathbb{P} E \rightarrow Z$ is generically one to one.

Note that the $G$-variety $\tau(X)$ is of course desingularized by $\mathbb{P} T X$, which is a special case of the above with $Q=P$.

\section{Triality and Exceptional Lie Algebras}

We begin with a review of division algebras via the Cayley-Dickson process in Section 3.1 and explicitly describe the derivations of $\mathbb{O}$ in Section 3.2 for later use. In Section 3.3 we review the triality principle for the octonions and its extension to all structurable algebras. We pause for a detour in Section 3.4, exploring the triality model for $\mathfrak{s o}_{4,4}$ in detail, which ends up being a 4ality. We review various constructions of the magic square in Section 3.5. We present a new result regarding the compatiblity of the Cayley-Dickson process and inclusions of triality algebras in Section 3.6, which at first glance appears to be trivial, but whose "obvious" generalization to structurable algebras is false. In Section 3.7 we study automorphisms related to the algebras in the magic square and the resulting magic squares of symmetric and trisymmetric spaces. In Section 3.8 we use our result in Section 3.6 to show how some towers of dual pairs naturally occur in series. In Section 3.9 we discuss quaternionic symmetric spaces, their relation to adjoint varieties and the conjecture of LeBrun and Salamon.

\subsection{Division Algebras}

A classical theorem of Hurwitz, published in 1898, asserts that there are only four normed division algebras over the field of real numbers: $\mathbb{R}$ itself, $\mathbb{C}$, the quaternions $\mathbb{H}$, and the octonions $\mathbb{O}$. A division algebra is an algebra without zero divisor and a normed division algebra is a division algebra endowed with a norm such that the norm of a product is the product of the norms.

Quaternions were discovered by Hamilton in 1843, and octonions shortly afterwards by Graves and Cayley. A nice way to define these algebras is to imitate the definition of complex numbers by a pair of real numbers. Let $\mathbb{A}$ be a real algebra endowed with a conjugation $x \mapsto \bar{x}$, such that $x+\bar{x}$ and $x \bar{x}=\bar{x} x$ are always real numbers (more precisely scalar multiples of the unit element), the later being positive when $x$ is nonzero. If $\mathbb{A}$ is alternative, i.e., any subalgebra generated by two elements is associative [5], then $\mathbb{A}$ is a normed algebra for the norm $\|x\|^{2}=x \bar{x}$.

One can then define a new algebra with conjugation $\mathbb{B}=\mathbb{A} \oplus \mathbb{A}$ by letting $(x, y)(z, t)=(x z-t \bar{y}, \bar{x} t+z y)$ with conjugation $\overline{(x, y)}=(\bar{x},-y)$. This is 
the Cayley-Dickson process. The new algebra $\mathbb{B}$ will be alternative, hence a normed algebra, iff $\mathbb{A}$ is associative.

In particular, $\mathbb{A}=\mathbb{C}$ gives the quaternion algebra $\mathbb{B}=\mathbb{H}$, which is associative, so the Cayley-Dickson process can be applied once more and $\mathbb{A}=\mathbb{H}$ gives the octonion algebra $\mathbb{B}=\mathbb{O}$. This algebra is no longer associative (although it is alternative, hence normed), and the process then fails to produce new normed algebras.

A useful variant of the Cayley-Dickson process is obtained by changing a sign in the formula for the product in $\mathbb{B}$, letting $(x, y)(z, t)=(x z+t \bar{y}, \bar{x} t+z y)$. The resulting algebra $\tilde{\mathbb{B}}$ is called split. It is no longer normed, but endowed with a nondegenerate quadratic form of signature $(a, a)$ compatible with the product. (Here $a$ denotes the dimension of $\mathbb{A}$.) In particular, it is a composition algebra. The algebra $\tilde{\mathbb{C}}$ of split complex numbers is the algebra $\mathbb{R} \oplus \mathbb{R}$ with termwise multiplication, while the split quaternion algebra $\mathbb{\mathbb { H }}$ is equivalent to $M_{2}(\mathbb{R})$.

\subsection{Derivations}

Any automorphism of $\mathbb{H}$ is inner, so that any derivation of $\mathbb{H}$ is of the form $L_{a}-R_{a}$ for some imaginary quaternion $a \in \operatorname{Im} \mathbb{H}$, where $L_{a}$ and $R_{a}$ denote the operators of left and right mulplication by $a$. In particular Der $\mathbb{H}=\operatorname{Im} \mathbb{H}=\mathfrak{s o}_{3}$.

The derivation algebra of $\mathbb{O}$ is the compact Lie algebra $\mathfrak{g}_{2}$. The algebra Der $\mathbb{H}=\mathfrak{s o}_{3}$ does not imbed in DerOO in a canonical way, but there is one prefered embedding for each decomposition $\mathbb{O}=\mathbb{H} \oplus e \mathbb{H}$, where the product in $\mathbb{O}$ is deduced from that in $\mathbb{H}$ through the Cayley-Dickson process. Indeed, for $\phi \in D e r \mathbb{H}$, the endomorphism $\tilde{\phi}$ of $\mathbb{O}$ defined by $\tilde{\phi}(x+e y)=\phi(x)+e \phi(y)$ is a derivation. Note that the subalgebra $\operatorname{Der}(\mathbb{O}, \mathbb{H})$ of the derivations of $\mathbb{O}$ stabilizing $\mathbb{H}$ is strictly greater than Der $\mathbb{H}$. It contains, for each imaginary quaternion $h$, the map $\psi_{h}(x+e y)=e(y h)$. This gives another copy of $\mathfrak{s o}_{3}$ in $\operatorname{Der}(\mathbb{O}, \mathbb{H})=\mathfrak{s o}_{3} \times \mathfrak{s o}_{3}$, and the restriction of these derivations to $e \mathbb{H}$ gives the full $\mathfrak{s o}_{4}=\mathfrak{s o}_{3} \times \mathfrak{s o}_{3}$. Explicitly, choose a standard basis $e_{0}=1, e_{1}, e_{2}$, $e_{3}=e_{1} e_{2}$ of $\mathbb{H}$, and let $e_{4}=e, e_{5}=e e_{1}, e_{6}=e e_{2}, e_{7}=e e_{3}$. Using this basis of $\mathbb{O}$, we obtain a matrix representation of $\mathfrak{g}_{2}=\operatorname{Der}(\mathbb{O})$ :

$$
\mathfrak{g}_{2}=\left(\begin{array}{cccccccc}
0 & 0 & 0 & 0 & 0 & 0 & 0 & 0 \\
0 & 0 & -\alpha_{2} & -\alpha_{3} & -\alpha_{4} & -\alpha_{5} & -\alpha_{6} & -\alpha_{7} \\
0 & \alpha_{2} & 0 & -\beta_{3} & -\beta_{4} & -\beta_{5} & -\beta_{6} & -\beta_{7} \\
0 & \alpha_{3} & \beta_{3} & 0 & \alpha_{6}+\beta_{5} & -\alpha_{5}-\beta_{4} & -\alpha_{4}+\beta_{7} & \alpha_{7}-\beta_{6} \\
0 & \alpha_{4} & \beta_{4} & -\alpha_{6}-\beta_{5} & 0 & -\gamma_{5} & -\gamma_{6} & -\gamma_{7} \\
0 & \alpha_{5} & \beta_{5} & \alpha_{5}+\beta_{4} & \gamma_{5} & 0 & -\alpha_{2}+\gamma_{7} & -\alpha_{3}-\gamma_{6} \\
0 & \alpha_{6} & \beta_{6} & \alpha_{4}-\beta_{7} & \gamma_{6} & \alpha_{2}-\gamma_{7} & 0 & \beta_{3}+\gamma_{5} \\
0 & \alpha_{7} & \beta_{7} & -\alpha_{7}+\beta_{6} & \gamma_{7} & \alpha_{3}+\gamma_{6} & -\beta_{3}-\gamma_{5} & 0
\end{array}\right) .
$$

It follows that the Lie sub-algebra $\operatorname{Der}(\mathbb{O}, \mathbb{H})$ may be written: 


$$
\operatorname{Der}(\mathbb{O}, \mathbb{H})=\left(\begin{array}{cccccccc}
0 & 0 & 0 & 0 & 0 & 0 & 0 & 0 \\
0 & 0 & -\alpha_{2} & -\alpha_{3} & 0 & 0 & 0 & 0 \\
0 & \alpha_{2} & 0 & -\beta_{3} & 0 & 0 & 0 & 0 \\
0 & \alpha_{3} & \beta_{3} & 0 & 0 & 0 & 0 & 0 \\
0 & 0 & 0 & 0 & 0 & -\gamma_{5} & -\gamma_{6} & -\gamma_{7} \\
0 & 0 & 0 & 0 & \gamma_{5} & 0 & -\alpha_{2}+\gamma_{7}-\alpha_{3}-\gamma_{6} \\
0 & 0 & 0 & 0 & \gamma_{6} & \alpha_{2}-\gamma_{7} & 0 & \beta_{3}+\gamma_{5} \\
0 & 0 & 0 & 0 & \gamma_{7} & \alpha_{3}+\gamma_{6}-\beta_{3}-\gamma_{5} & 0
\end{array}\right) .
$$

Our first type of derivations in $\operatorname{Der}(\mathbb{O}, \mathbb{H})$ corresponds to those matrices for which $\gamma_{5}=\gamma_{6}=\gamma_{7}=0$, and the second type to those for which $\alpha_{2}=$ $\alpha_{3}=\beta_{3}=0$. These two copies of $\mathfrak{s o}_{3}$ commute. Finally, the south-east corner of the matrix above gives a copy of $\mathfrak{s o}_{4}$.

\subsection{Triality}

\subsubsection{Cartan's Triality}

The Dynkin diagram of type $D_{4}$ is the only one with a threefold symmetry:

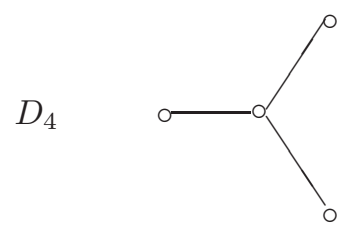

Symmetries of Dynkin diagrams detect outer automorphisms of the corresponding complex (or split) Lie algebras, and in the case of $D_{4}$ this is closely related with the algebra structure of the octonions. Indeed, let

$$
T(\mathbb{O})=\left\{\left(\mathrm{U}_{1}, \mathrm{U}_{2}, \mathrm{U}_{3}\right) \in \mathrm{SO}(\mathbb{O})^{3} \mid \mathrm{U}_{1}(x y)=\mathrm{U}_{2}(x) \mathrm{U}_{3}(y) \forall x, y \in \mathbb{O}\right\} .
$$

This group contains $\operatorname{Aut}(\mathbb{O})=G_{2}$ and has three projections $\pi_{1}, \pi_{2}, \pi_{3}$ on $\mathrm{SO}(\mathbb{O})$, hence three representations of dimension eight. The following theorem is due to Elie Cartan and can be found page 370 of his paper [10] from 1925. Define $\operatorname{Spin}_{8}$ to be the compact, simply connected Lie group with Lie algebra $\mathfrak{s o}_{8}$.

Theorem 3.1. The group $T(\mathbb{O})$ is $\mathrm{Spin}_{8}$, and each projection $\pi_{i}$ is a twofold covering of $\mathrm{SO}_{8}$. The three corresponding eight dimensional representations of $\mathrm{Spin}_{8}$ are not equivalent.

With this description of $\operatorname{Spin}_{8}$ in hand, it is easy to define an action of $\mathfrak{S}_{3}$ by outer automorphisms. Indeed, since $\mathbb{O}$ is alternative and $\bar{x}=-x$ modulo the unit element of $\mathbb{O}$, the relation $\bar{x}(x y)=(\bar{x} x) y$ holds. (This and similar 
identities are due to Moufang, see [39].) A little computation then shows that the identities

$$
\mathrm{U}_{1}(x y)=\mathrm{U}_{2}(x) \mathrm{U}_{3}(y), \quad \mathrm{U}_{2}(x y)=\mathrm{U}_{1}(x) \overline{\mathrm{U}_{3}(\bar{y})}, \quad \mathrm{U}_{3}(x y)=\overline{\mathrm{U}_{2}(\bar{x})} \mathrm{U}_{1}(y)
$$

are equivalent. If we denote by $\tau \in \mathrm{SO}(\mathbb{O})$ the conjugation anti-automorphism, this implies that we can define automorphisms $s$ and $t$ of $\operatorname{Spin}_{8}$ by letting

$$
\begin{aligned}
& s\left(\mathrm{U}_{1}, \mathrm{U}_{2}, \mathrm{U}_{3}\right)=\left(\mathrm{U}_{2}, \mathrm{U}_{1}, \tau \circ \mathrm{U}_{3} \circ \tau\right), \\
& t\left(\mathrm{U}_{1}, \mathrm{U}_{2}, \mathrm{U}_{3}\right)=\left(\tau \circ \mathrm{U}_{1} \circ \tau, \tau \circ \mathrm{U}_{3} \circ \tau, \tau \circ \mathrm{U}_{2} \circ \tau\right) .
\end{aligned}
$$

These automorphisms are not inner, since they exchange the three inequivalent eight dimensional representations of $\operatorname{Spin}_{8}$. Moreover, we have $s^{2}=t^{2}=1$ and $s t s=t s t$, so that $s$ and $t$ generate a group isomorphic to $\mathfrak{S}_{3}$, which is the full group of outer automorphisms of $\operatorname{Spin}_{8}$.

Note that at the level of Lie algebras, Cartan's theorem above implies the infinitesimal triality principle, which we state as

$$
\mathfrak{t}(\mathbb{O}):=\left\{\left(u_{1}, u_{2}, u_{3}\right) \in \mathfrak{s o}(\mathbb{O})^{3} \mid u_{1}(x y)=u_{2}(x) y+x u_{3}(y) \forall x, y \in \mathbb{O}\right\}=\mathfrak{s o}_{8} .
$$

This means that if we fix $u_{1}$ for example, there is a unique pair of skewsymmetric endomorphism of $(\mathbb{O}$ such that the above relation holds.

\subsubsection{Triality Algebras}

Allison and Faulkner [3], following work of Allison [2] and Kantor [50] define the following vast generalization of the triality principle. Let $\mathcal{A}$ be any algebra with unit and involution $a \mapsto \bar{a}$ over a ring of scalars. Let $\operatorname{Im} \mathcal{A}=\{a \in \mathcal{A}, \bar{a}=$ $-a\}$. Define the triality algebra of $\mathcal{A}$

$$
\mathfrak{t}(\mathcal{A}):=\left\{T=\left(T_{1}, T_{2}, T_{3}\right) \in \mathfrak{g l}(\mathcal{A})^{\oplus 3} \mid \overline{T_{1}}(a b)=T_{2}(a) b+a T_{3}(b) \quad \forall a, b \in \mathcal{A}\right\},
$$

with $\overline{T_{1}}(a)=\overline{T_{1}(\bar{a})}$. This is a Lie subalgebra of $\mathfrak{g l}(\mathcal{A})^{\oplus 3}$. In [3] it is call the set of partially related Lie triples of $\mathcal{A}$. If 3 is invertible in the ring of scalars, they provide (Corollary 3.5) a general description of $\mathfrak{t}(\mathcal{A})$, which in the case where $\mathcal{A}$ is alternative gives

$$
\begin{aligned}
\mathfrak{t}(\mathcal{A}) & =\left\{\left(D+L_{s}-R_{t}, D+L_{t}-R_{r}, D+L_{r}-R_{s}\right) \mid D \in \operatorname{Der}(\mathcal{A}),\right. \\
& =\operatorname{Der}(\mathcal{A}) \oplus \operatorname{Im}(\mathcal{A})^{\oplus 2} .
\end{aligned}
$$

If $D$ is a derivation, $(D, D, D) \in \mathfrak{t}(\mathcal{A})$ because $\bar{D}=D$. Also, recall that $\mathcal{A}$ being alternative means that any subalgebra generated by two elements is associative, or equivalently, that the associator

$$
\{x, y, z\}:=(x y) z-x(y z), \quad x, y, z \in \mathcal{A},
$$


is a skew-symmetric trilinear function. In particular, we have $\{x, y, z\}=$ $\{z, x, y\}$, which can be rewritten as $(x y) z+z(x y)=x(y z)+(z x) y$. If $z$ is imaginary, this shows that $\left(-L_{z}-R_{z}, L_{z}, R_{z}\right)$ is in $\mathfrak{t}(\mathcal{A})$. Using the triality automorphisms defined above, one gets that $\left(L_{s}-R_{t}, L_{t}-R_{r}, L_{r}-R_{s}\right)$ belongs to $\mathfrak{t}(\mathcal{A})$ as soon as $r, s, t$ are imaginary with $r+s+t=0$.

For composition algebras, we get

$$
\begin{gathered}
\mathfrak{t}(\mathbb{R})=0, \\
\mathfrak{t}(\mathbb{C})=\mathbb{R}^{2}, \mathfrak{t}(\tilde{\mathbb{C}})=\mathbb{R}^{2}, \\
\mathfrak{t}(\mathbb{H})=\left(\mathfrak{s o}_{3}\right)^{\oplus 3}, \mathfrak{t}(\tilde{\mathbb{H}})=\left(\mathfrak{s l}_{2}\right)^{\oplus 3}, \\
\mathfrak{t}(\mathbb{O})=\mathfrak{s o}_{8}, \mathfrak{t}(\tilde{\mathbb{O}})=\mathfrak{s o}_{4,4} .
\end{gathered}
$$

\subsection{Triality and $\mathfrak{s o}_{4,4}: 4$-ality}

An interesting version of triality holds for $\mathfrak{s o}_{4,4}$, the split version of $\mathfrak{s o}_{8}$. We use a model is related to the fourfold symmetry of the extended Dynkin diagram $\tilde{D}_{4}$ :

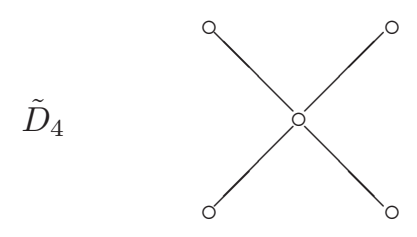

Choose four real two-dimensional vector spaces $A, B, C, D$ and non-degenerate two-forms on each of them. Then

$$
\mathfrak{s o}_{4,4}=\mathfrak{s l}(A) \times \mathfrak{s l}(B) \times \mathfrak{s l}(C) \times \mathfrak{s l}(D) \oplus(A \otimes B \otimes C \otimes D) .
$$

In this model, $\mathfrak{s l}(A) \times \mathfrak{s l}(B) \times \mathfrak{s l}(C) \times \mathfrak{s l}(D)$ is a sub-Lie algebra and acts on $A \otimes B \otimes C \otimes D$ in the obvious way. The bracket of two vectors in $A \otimes B \otimes C \otimes D$ can be defined as follows. Choose non-degenerate skew-symmetric two forms on $A, B, C$ and $D$. We denote these forms by the same letter $\omega$. We have then natural isomorphisms $S^{2} A \rightarrow \mathfrak{s l}(A)$, and so on, by sending a square $a^{2}$, where $a \in A$, to the endomorphism $a^{\prime} \mapsto \omega\left(a, a^{\prime}\right) a$. With this understood, we let

$$
\begin{aligned}
& {\left[a \otimes b \otimes c \otimes d, a^{\prime} \otimes b^{\prime} \otimes c^{\prime} \otimes d^{\prime}\right]} \\
& =\omega\left(b, b^{\prime}\right) \omega\left(c, c^{\prime}\right) \omega\left(d, d^{\prime}\right) a a^{\prime}+\omega\left(a, a^{\prime}\right) \omega\left(c, c^{\prime}\right) \omega\left(d, d^{\prime}\right) b b^{\prime}+ \\
& \quad+\omega\left(a, a^{\prime}\right) \omega\left(b, b^{\prime}\right) \omega\left(d, d^{\prime}\right) c c^{\prime}+\omega\left(a, a^{\prime}\right) \omega\left(b, b^{\prime}\right) \omega\left(c, c^{\prime}\right) d d^{\prime} .
\end{aligned}
$$

One can then check that the Jacobi identity holds, so that we obtain a Lie algebra which can easily be identified with $\mathfrak{s o}_{4,4}$. One could also take in $\mathfrak{s o}_{4,4}$, once a Cartan subalgebra and a set of positive roots have been chosen, the product of the four copies of $\mathfrak{s l}_{2}$ generated by the three simple roots and by 
the highest root. These four copies commute, and the sum of the other root spaces forms a module over their product. It is then routine to identify this module with the tensor product of the natural representations.

What is particularily nice with this model is that we can explicitly define three inequivalent eight dimensional representations, which we denote by

$$
\begin{aligned}
& \tilde{\mathbb{O}}_{1}=(A \otimes B) \oplus(C \otimes D), \\
& \tilde{\mathbb{O}}_{2}=(A \otimes C) \oplus(B \otimes D), \\
& \tilde{\mathbb{O}}_{3}=(A \otimes D) \oplus(B \otimes C) .
\end{aligned}
$$

The action of $\mathfrak{s o}_{4,4}$ is easy to describe: the action of $\mathfrak{s l}(A) \times \mathfrak{s l}(B) \times \mathfrak{s l}(C) \times \mathfrak{s l}(D)$ is the obvious one, while that of $A \otimes B \otimes C \otimes D$ on $\tilde{\mathbb{O}}_{1}$, for example, is defined by

$$
a \otimes b \otimes c \otimes d .\left(a^{\prime} \otimes b^{\prime}+c^{\prime} \otimes d^{\prime}\right)=\omega\left(c, c^{\prime}\right) \omega\left(d, d^{\prime}\right) a \otimes b+\omega\left(a, a^{\prime}\right) \omega\left(b, b^{\prime}\right) c \otimes d .
$$

To see that we get three inequivalent representations, we can describe the root system of $\mathfrak{s o}_{4,4}$ thanks to the previous model, and check that the highest weights are distinct. Moreover, once isomorphisms of $A, B, C$ and $D$ with a fixed two-dimensional vector space have been chosen, there is an obvious action of the symmetric group $\mathfrak{S}_{4}$ on $\mathfrak{s o}_{4,4}$ and its triplet of representations. The resulting group of outer automorphisms is only $\mathfrak{S}_{3}$, of course, which in this model appears as the quotient of $\mathfrak{S}_{4}$ by the normal subgroup of order four whose three nontrivial elements are products of disjoint two-cycles (recall that the alternating group $\mathcal{A}_{4}$ is not simple!). (See [55] for a geometric interpretation of the $\mathfrak{s o}_{4,4}$ triality, and an application to the construction of a minimal unitary representation.) Note also that the subalgebra of fixed points of the $\mathfrak{S}_{3}$-action (defined by the permutations of $A, B, C$ ) on $\mathfrak{s o}_{4,4}$ is

$$
\tilde{\mathfrak{g}}_{2}=\mathfrak{s l}(A) \times \mathfrak{s l}(D) \oplus\left(S^{3} A \otimes D\right),
$$

the split form of $\mathfrak{g}_{2}$.

As pointed out by J. Wolf in [34], one can twist this $\mathfrak{S}_{3}$-action so as to get a different fixed point subalgebra. We define automorphisms $\tau$ and $\tau^{\prime}$ of $\mathfrak{s o}_{4,4}$, acting on $(M, N, P, Q) \in \mathfrak{s l}(A) \times \mathfrak{s l}(B) \times \mathfrak{s l}(C) \times \mathfrak{s l}(D)$ and on $a \otimes b \otimes c \otimes d \in A \otimes B \otimes C \otimes D$ in the following way:

$$
\begin{array}{ll}
\tau(M, N, P, Q)=(P, M, N, Q), & \tau(a \otimes b \otimes c \otimes d)=a \otimes b \otimes c \otimes d, \\
\tau^{\prime}(M, N, P, Q)=(P, M, N, a d(T) Q), & \tau^{\prime}(a \otimes b \otimes c \otimes d)=c \otimes a \otimes b \otimes T(d),
\end{array}
$$

where $T$ is a rotation matrix of order three (so that $T^{2}+T+1=0$ ). The fixed point set of $\tau^{\prime}$ is $\mathfrak{s l}(A) \times \mathbb{R} H \oplus M$, where $H=1+2 T$ and $M$ is the space of fixed points of $\tau^{\prime}$ inside $A \otimes B \otimes C \otimes D$. Write such a tensor as $X \otimes e+Y \otimes T e$ for some nonzero vector $e$ in $D$, where $X$ and $Y$ belong to $A \otimes B \otimes C$. It is fixed by $\tau^{\prime}$ if and only if $X=-\tau(Y)$ and $\left(1+\tau+\tau^{2}\right)(Y)=0$, which means that $Y=Z-\tau(Z)$ for some $Z$, and then $X=\tau^{2}(Z)-\tau(Z)$. In particular $M$ has dimension 4 and it is easy to see that the space of fixed points of $\tau^{\prime}$ is a copy of $\mathfrak{s l}_{3}$. 


\subsection{The Magic Square}

\subsubsection{Jordan Algebras}

The relation between exceptional Lie groups and normed division algebras, especially the octonion algebra, was first noticed by E. Cartan, at least as early as 1908 (see the encyclopedia article for the French version of the Mathematische Wissenschaften). Cartan observed that the automorphism group of $\mathbb{O}$ is the compact group $G_{2}$. In 1950, Chevalley and Schafer obtained the compact group $F_{4}$ as the automorphism group of the Jordan algebra

$$
\mathcal{J}_{3}(\mathbb{O}):=\left\{\left(\begin{array}{lll}
\frac{r_{1}}{x_{3}} & x_{2} \\
\overline{x_{2}} & \frac{r_{2}}{x_{1}} & x_{1}
\end{array}\right) \mid r_{i} \in \mathbb{R}, x_{j} \in \mathbb{O}\right\} .
$$

This is an algebra with product $A \cdot B=\frac{1}{2}(A B+B A)$. It is a commutative but nonassociative algebra, in which the characteristic identity $A^{2}(A B)=A\left(A^{2} B\right)$ of Jordan algebras holds. By remarks in [12], it appears Cartan was aware of this construction at least as early as 1939 .

Jordan algebras were introduced in the 1930's by Jordan, von Neumann and Wigner as a natural mathematical framework for quantum theory. Any associative algebra (over a field of characteristic not equal to 2) can be considered as a Jordan algebra for the symmetrized matrix product $a \cdot b=\frac{1}{2}(a b+$ $b a)$. A Jordan algebra is said to be exceptional if it cannot be embedded as a Jordan subalgebra of an associative algebra. The algebra $\mathcal{J}_{3}(\mathbb{O})$ is an exceptional Jordan algebra. Moreover, if $A$ is an algebra such that $\mathcal{J}_{n}(A)$, with its symmetrized product, is a Jordan algebra, then $A$ must be alternative if $n \geq 3$, and associative if $n \geq 4$.

The closed orbits $X \subset \mathcal{J}_{n}(\mathbb{A})$, with $\mathbb{A}$ the complexification of a division algebra are exactly the Scorza varieties while the Severi varieties are exactly the varieties $X \subset \mathcal{J}_{3}(\mathbb{A})$, see [85]. The close relationship between Scorza varieties and Jordan algebras, which is not explained in [85], is investigated in [16].

\subsubsection{The Freudenthal-Tits Construction}

The discovery that $F_{4}=\operatorname{Aut} \mathcal{J}_{3}(\mathbb{O})$ led several authors (including Freudenthal, Tits, Vinberg, Jacobson, Springer) to study the relations between exceptional groups and Jordan algebras. Freudenthal and Tits obtained a unified construction of all compact exceptional Lie algebras in the following way: take two normed division algebras $\mathbb{A}$ and $\mathbb{B}$, and let

$$
\mathfrak{g}(\mathbb{A}, \mathbb{B}):=\operatorname{Der} \mathbb{A} \oplus\left(\operatorname{Im} \mathbb{A} \otimes \mathcal{J}_{3}(\mathbb{B})_{0}\right) \oplus \operatorname{Der} \mathcal{J}_{3}(\mathbb{B}),
$$

where $\mathcal{J}_{3}(\mathbb{B})_{0} \subset \mathcal{J}_{3}(\mathbb{B})$ is the hyperplane of traceless matrices. One can then define a Lie algebra structure on $\mathfrak{g}(\mathbb{A}, \mathbb{B})$, with $\operatorname{Der} \mathbb{A} \oplus \operatorname{Der} \mathcal{J}_{3}(\mathbb{B})$ as a Lie subalgebra acting on $\operatorname{Im} \mathbb{A} \otimes \mathcal{J}_{3}(\mathbb{B})_{0}$ in a natural way [79]. The result of this construction is the Freudenthal-Tits magic square of Lie algebras: 


\begin{tabular}{|c|cccc|}
\hline & $\mathbb{R}$ & $\mathbb{C}$ & $\mathbb{H}$ & $\mathbb{O}$ \\
\hline $\mathbb{R}$ & $\mathfrak{s u}_{2}$ & $\mathfrak{s u}_{3}$ & $\mathfrak{s p}_{6}$ & $\mathfrak{f}_{4}$ \\
$\mathbb{C}$ & $\mathfrak{s u}_{3}$ & $\mathfrak{s u}_{3} \times \mathfrak{s u}_{3}$ & $\mathfrak{s u}_{6}$ & $\mathfrak{e}_{6}$ \\
$\mathbb{H}$ & $\mathfrak{s p}_{6}$ & $\mathfrak{s u}_{6}$ & $\mathfrak{s o}_{12}$ & $\mathfrak{e}_{7}$ \\
$\mathbb{O}$ & $\mathfrak{f}_{4}$ & $\mathfrak{e}_{6}$ & $\mathfrak{e}_{7}$ & $\mathfrak{e}_{8}$ \\
\hline
\end{tabular}

Let $\mathbb{B}=\underline{0}$ denote the zero algebra, so $\mathcal{J}_{n}(\underline{0}) \simeq \mathbb{C}^{n}$ is the diagonal matrices. Let $\Delta$ be something slightly smaller, so that $\mathcal{J}_{n}(\Delta) \subset \mathcal{J}_{n}(\underline{0})$ consists of the scalar matrices. With these conventions, we obtain a magic rectangle:

\begin{tabular}{|c|cccccc|}
\hline & $\Delta$ & $\underline{0}$ & $\mathbb{R}$ & $\mathbb{C}$ & $\mathbb{H}$ & $\mathbb{O}$ \\
\hline $\mathbb{R}$ & 0 & 0 & $\mathfrak{s u}_{2}$ & $\mathfrak{s u}_{3}$ & $\mathfrak{s p}_{6}$ & $\mathfrak{f}_{4}$ \\
$\mathbb{C}$ & 0 & 0 & $\mathfrak{s u}_{3}$ & $\mathfrak{s u}_{3} \times \mathfrak{s u}_{3}$ & $\mathfrak{s u}_{6}$ & $\mathfrak{e}_{6}$ \\
$\mathbb{H}$ & $\mathfrak{s u}_{2}$ & $\left(\mathfrak{s u}_{2}\right)^{\oplus 3}$ & $\mathfrak{s p}_{6}$ & $\mathfrak{s u}_{6}$ & $\mathfrak{s o}_{12}$ & $\mathfrak{e}_{7}$ \\
$\mathbb{O}$ & $\mathfrak{g}_{2}$ & $\mathfrak{s o}_{8}$ & $\mathfrak{f}_{4}$ & $\mathfrak{e}_{6}$ & $\mathfrak{e}_{7}$ & $\mathfrak{e}_{8}$ \\
\hline
\end{tabular}

\subsubsection{The Allison Construction}

While it is impressive that the vector space $\mathfrak{g}(\mathbb{A}, \mathbb{B})$ is a Lie algebra, the symmetry of the square appears to be miraculous. Vinberg, in 1966, obtained a symmetric construction (see [72] for an exposition). We present a variant of the Vinberg construction first discovered by Allison [2], then rediscovered independently in more geometric form by Dadok and Harvey [23] (who, after discovering Allison's work decided not to publish their manuscript), Barton and Sudbery [7], and ourselves [62].

Allison shows that given an arbitrary structurable algebra $\mathcal{A}$, one can put a Lie algebra structure on

$$
\mathfrak{t}(\mathcal{A}) \oplus \mathcal{A}^{\oplus 3}
$$

Allison observes moreover that the algebra $\mathcal{A}=\mathbb{A} \otimes \mathbb{B}$ with $\mathbb{A}, \mathbb{B}$ division algebras is structurable and his construction applied to them yields the triality model, see $[2,3]$.

Using the Allison construction, the magic square may be described as follows:

$$
\mathfrak{g}(\mathbb{A}, \mathbb{B})=\mathfrak{t}(\mathbb{A}) \times \mathfrak{t}(\mathbb{B}) \oplus\left(\mathbb{A}_{1} \otimes \mathbb{B}_{1}\right) \oplus\left(\mathbb{A}_{2} \otimes \mathbb{B}_{2}\right) \oplus\left(\mathbb{A}_{3} \otimes \mathbb{B}_{3}\right) .
$$

This bracket is defined so that $\mathfrak{t}(\mathbb{A}) \times \mathfrak{t}(\mathbb{B})$ is a Lie subalgebra, acting on each $\mathbb{A}_{i} \otimes \mathbb{B}_{i}$ in the natural way. The bracket of an element $a_{1} \otimes b_{1} \in \mathbb{A}_{1} \otimes \mathbb{B}_{1}$ with $a_{2} \otimes b_{2} \in \mathbb{A}_{2} \otimes \mathbb{B}_{2}$ is simply $a_{1} a_{2} \otimes b_{1} b_{2}$, considered as an element of $\mathbb{A}_{3} \otimes \mathbb{B}_{3}$. This is the general rule for taking the bracket of an element of $\mathbb{A}_{i} \otimes \mathbb{B}_{i}$ with one of $\mathbb{A}_{j} \otimes \mathbb{B}_{j}$, although there are some slight twists whose details can be found in [3] or [62]. Finally, the bracket of two elements in $\mathbb{A}_{i} \otimes \mathbb{B}_{i}$ is defined by the quadratic forms on $\mathbb{A}_{i}, \mathbb{B}_{i}$ and the natural maps $\Psi_{i}: \wedge^{2} \mathbb{A}_{i} \rightarrow \mathfrak{t}(\mathbb{A})$ 
obtained by dualizing the action of $\mathfrak{t}(\mathbb{A})$ on $\mathbb{A}_{i}$, which can be seen as a map $\mathfrak{t}(\mathbb{A}) \rightarrow \wedge^{2} \mathbb{A}_{i}$. (Note that $\mathfrak{t}(\mathbb{A})$ is always reductive, hence isomorphic to its dual as a $\mathfrak{t}(\mathbb{A})$-module.) The key formulas that ensure that the Jacobi identities hold in $\mathfrak{g}(\mathbb{A}, \mathbb{B})$ are then the following, which we state only for $\Psi_{1}$ :

$$
\begin{aligned}
& \Psi_{1}(u \wedge v)_{1} x=Q(u, x) v-Q(v, x) u \\
& \Psi_{1}(u \wedge v)_{2} x=\frac{1}{2}(\bar{v}(u x)-\bar{u}(v x)) \\
& \Psi_{1}(u \wedge v)_{3} x=\frac{1}{2}((x u) \bar{v}-(x v) \bar{u}) .
\end{aligned}
$$

These formulas are classical in the study of triality, that is for $\mathbb{A}=\mathbb{O}$. It is easy to check that in the other cases, which are much easier, we can arrange so that they also hold.

Proposition 3.2. The real Lie algebra $\mathfrak{g}(\mathbb{A}, \mathbb{B})$ is compact, while $\mathfrak{g}(\tilde{\mathbb{A}}, \tilde{\mathbb{B}})$ is split.

Proof. Let $K_{\mathbb{A}}$ be the non-degenerate quadratic form on $\mathfrak{t}(\mathbb{A})$ which we used in the construction of $\mathfrak{g}(\mathbb{A}, \mathbb{B})$. For $\mathbb{A}=\mathbb{O}$, this form is the Killing form on $\mathfrak{s o}_{8}$ and is therefore negative definite; this is also true for the other division algebras. Now it is easy to check that the quadratic form

$$
\mathcal{Q}:=K_{\mathbb{A}}+K_{\mathbb{B}}-\sum_{i=1}^{3} Q_{\mathbb{A}_{i}} \otimes Q_{\mathbb{B}_{i}}
$$

is invariant and negative definite. Since $\mathfrak{g}(\mathbb{A}, \mathbb{B})$ is simple (except for $\mathbb{A}=$ $\mathbb{B}=\mathbb{C}$ ), this invariant form must be proportional to the Killing form, which is therefore negative definite as well (it cannot be positive definite!). Thus $\mathfrak{g}(\mathbb{A}, \mathbb{B})$ is compact.

Finally $\mathfrak{g}(\tilde{\mathbb{A}}, \tilde{\mathbb{B}})$ is clearly split since both $\mathfrak{t}(\tilde{\mathbb{A}})$ and $\mathfrak{t}(\tilde{\mathbb{B}})$ are split, and their product is a maximal rank subalgebra of $\mathfrak{g}(\mathbb{A}, \mathbb{B})$.

\subsection{Inclusions}

It is not at all obvious that an inclusion $\mathbb{B} \subset \mathbb{B}^{\prime}$ of composition algebras induces an inclusion $\mathfrak{g}(\mathbb{A}, \mathbb{B}) \subset \mathfrak{g}\left(\mathbb{A}, \mathbb{B}^{\prime}\right)$ of Lie algebras. In fact the Allison-Faulkner process applied to structurable algebras $\mathcal{A} \subset \mathcal{B}$ does not imply inclusions $\mathfrak{g}(\mathcal{A}) \subset \mathfrak{g}(\mathcal{B})$. To discuss this problem in our situation we may assume that $\mathbb{B}^{\prime}=\mathbb{B} \oplus e \mathbb{B}$ is deduced from $\mathbb{B}$ via the Cayley-Dickson process.

Theorem 3.3. There is a unique embedding of Lie algebras $\mathfrak{t}(\mathbb{B}) \hookrightarrow \mathfrak{t}\left(\mathbb{B}^{\prime}\right)$ that makes $\mathfrak{g}(\mathbb{A}, \mathbb{B})$ a Lie subalgebra of $\mathfrak{g}\left(\mathbb{A}, \mathbb{B}^{\prime}\right)$.

If $\mathbb{B}^{\prime}$ is deduced from $\mathbb{B}$ by the Cayley-Dickson process, the relevant embedding of $\mathfrak{t}(\mathbb{B})$ inside $\mathfrak{t}\left(\mathbb{B}^{\prime}\right)$ is

$$
\mathfrak{t}(\mathbb{B}) \simeq\left\{\left(\mathrm{U}_{1}, \mathrm{U}_{2}, \mathrm{U}_{3}\right) \in \mathfrak{t}\left(\mathbb{B}^{\prime}\right) \mid \mathrm{U}_{i}(\mathbb{B}) \subset \mathbb{B}\right\} \subset \mathfrak{t}\left(\mathbb{B}^{\prime}\right) .
$$


Proof. We want the inclusion $\mathfrak{g}(\mathbb{A}, \mathbb{B}) \hookrightarrow \mathfrak{g}\left(\mathbb{A}, \mathbb{B}^{\prime}\right)$ to be a morphism of Lie algebras. It is easy to see that the only non-obvious verification to be made is that the bracket of two elements in $\mathbb{A}_{i} \otimes \mathbb{B}_{i}$ is the same in $\mathfrak{g}(\mathbb{A}, \mathbb{B})$ as in $\mathfrak{g}\left(\mathbb{A}, \mathbb{B}^{\prime}\right)$, which amounts to proving that we have a commutative diagram

$$
\begin{array}{ccc}
\wedge^{2}\left(\mathbb{A}_{i} \otimes \mathbb{B}_{i}\right) & \longrightarrow \mathfrak{t}(\mathbb{A}) \times \mathfrak{t}(\mathbb{B}) \\
\downarrow & & \downarrow \\
\wedge^{2}\left(\mathbb{A}_{i} \otimes \mathbb{B}_{i}^{\prime}\right) & \longrightarrow & \mathfrak{t}(\mathbb{A}) \times \mathfrak{t}\left(\mathbb{B}^{\prime}\right) .
\end{array}
$$

Since the norm on $\mathbb{B}$ is the restriction of the norm on $\mathbb{B}^{\prime}$, it is actually enough to prove the commutativity of the following diagram:

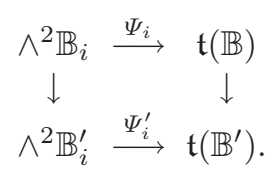

We must check this for all $i$, so we consider the sum $\Psi_{\mathbb{B}}: \wedge^{2} \mathbb{B}_{1} \oplus \wedge^{2}$ $\mathbb{B}_{2} \oplus \wedge^{2} \mathbb{B}_{3} \longrightarrow \mathfrak{t}(\mathbb{B})$. Note that $\Psi_{\mathbb{B}}$ is dual to the action, hence surjective since the action map is injective by definition. So the commutativity of the diagram above for $i=1,2,3$ completely determines the inclusion of $\mathfrak{t}(\mathbb{B})$ inside $\mathfrak{t}\left(\mathbb{B}^{\prime}\right)$. For this inclusion map to be well-defined, we need a compatibility condition, namely that $\operatorname{Ker} \Psi_{\mathbb{B}} \subset \operatorname{Ker} \Psi_{\mathbb{B}^{\prime}}$. Everything being trivial for $\mathbb{B}=\mathbb{R}$, we will check that $\operatorname{Ker} \Psi_{\mathbb{C}} \subset \operatorname{Ker} \Psi_{\mathbb{H}} \subset \operatorname{Ker} \Psi_{\mathbb{O}}$ and deduce the relevant embeddings $\mathfrak{t}(\mathbb{C}) \subset \mathfrak{t}(\mathbb{H}) \subset \mathfrak{t}(\mathbb{O})$.

We begin with $\Psi_{\mathbb{C}}$, which is dual to the inclusion map

$$
\mathfrak{t}(\mathbb{C})=\left\{(a, b, c) \in \mathbb{R}^{3}, a=b+c\right\} \subset \wedge^{2} \mathbb{C}_{1} \oplus \wedge^{2} \mathbb{C}_{2} \oplus \wedge^{2} \mathbb{C}_{3},
$$

where for example $a \in \mathbb{R}$ maps to the skew-symmetric endomorphism $\left(\begin{array}{cc}0 & a \\ -a & 0\end{array}\right)$ of $\mathbb{C}_{1}$. On $\mathfrak{t}(\mathbb{C})$ we use the restriction of the canonical norm of $\mathbb{R}^{3}$, and we find that the dual map is

$\Psi_{\mathbb{C}}:\left(\left(\begin{array}{cc}0 & a \\ -a & 0\end{array}\right),\left(\begin{array}{cc}0 & b \\ -b & 0\end{array}\right),\left(\begin{array}{cc}0 & c \\ -c & 0\end{array}\right)\right) \mapsto\left(\frac{2 a+b+c}{3}, \frac{a+2 b-c}{3}, \frac{a-b+2 c}{3}\right)$,

whose kernel is generated by $\left(\left(\begin{array}{cc}0 & 1 \\ -1 & 0\end{array}\right),\left(\begin{array}{cc}0 & -1 \\ 1 & 0\end{array}\right),\left(\begin{array}{cc}0 & -1 \\ 1 & 0\end{array}\right)\right)$.

Now to compute $\Psi_{\mathbb{H}}$, using that $\operatorname{Der}(\mathbb{H}) \simeq \operatorname{Im\mathbb {H}}$ we may describe $\mathfrak{t}(\mathbb{H})$ as

$$
\mathfrak{t}(\mathbb{H})=\left\{\left(L_{a}+R_{b}, L_{a}-R_{c}, L_{c}+R_{b}\right) \mid a, b, c \in \operatorname{Im} \mathbb{H}\right\} .
$$

Every skew-symmetric endomorphism of $\mathbb{H}$ is of the form $L_{x}+R_{y}$ for some imaginary quaternions $x$ and $y$, and the norm defined by the Killing form is $\left\|L_{x}+R_{y}\right\|^{2}=\|x\|^{2}+\|y\|^{2}$. With the induced norm on $\mathfrak{t}(\mathbb{H})$ we compute that

$$
\begin{aligned}
\Psi_{\mathbb{H}} & :\left(L_{x_{1}}+R_{y_{1}}, L_{x_{2}}+R_{y_{2}}, L_{x_{3}}+R_{y_{3}}\right) \\
& \mapsto\left(L_{x_{1}+x_{3}}+R_{y_{1}+y_{2}}, L_{x_{1}+x_{3}}-R_{x_{2}-y_{3}}, L_{x_{2}-y_{3}}+R_{y_{1}+y_{2}}\right) .
\end{aligned}
$$

Its kernel is $\left\{\left(L_{x}+R_{y}, L_{z}-R_{y},-L_{x}+R_{z}\right) \mid x, y, z \in \operatorname{Im} \mathbb{H}\right\}$. 
Finally, the computation of $\Psi_{\mathbb{O}}$ is an immediate consequence of the triality principle. Indeed, we know that each projection $\mathfrak{t}(\mathbb{O}) \rightarrow \wedge^{2} \mathbb{O}_{i}$ is an isomorphism. If we denote by $\left(U, U^{\prime}, U^{\prime \prime}\right)$ the elements of $\mathfrak{t}(\mathbb{O})$, this means that such a triplet is uniquely determined by $U, U^{\prime}$ or $U^{\prime \prime}$. We deduce that

$$
\Psi_{\mathbb{O}}:\left(\mathrm{U}_{1}, \mathrm{U}_{2}^{\prime}, \mathrm{U}_{3}^{\prime \prime}\right) \mapsto\left(\mathrm{U}_{1}+\mathrm{U}_{2}+\mathrm{U}_{3}, \mathrm{U}_{1}^{\prime}+\mathrm{U}_{2}^{\prime}+\mathrm{U}_{3}^{\prime}, \mathrm{U}_{1}^{\prime \prime}+\mathrm{U}_{2}^{\prime \prime}+\mathrm{U}_{3}^{\prime \prime}\right) .
$$

We are ready to check our compatibility conditions. First note that the endomorphism $\left(\begin{array}{cc}0 & 1 \\ -1 & 0\end{array}\right)$ of $\mathbb{C}$, when extended to $\mathbb{H}$ in the obvious way, is $1 \wedge i=$ $\frac{1}{2}\left(L_{i}+R_{i}\right)$. Taking $x=y=-z=i$ in our description of $\operatorname{Ker} \Psi_{\mathbb{H}}$, we see that it contains $\operatorname{Ker} \Psi_{\mathbb{C}}$, as required, and that the induced inclusion $\mathfrak{t}(\mathbb{C}) \subset \mathfrak{t}(\mathbb{H})$ is given by

$$
(b, c) \in \mathbb{R}^{2} \mapsto\left(L_{(b+2 c) i}+R_{(2 b+c) i}, L_{(b+2 c) i}+R_{(c-b) i}, L_{(b-c) i}+R_{(2 b+c) i}\right) .
$$

Now take $\left(L_{x}+R_{y}, L_{z}-R_{y},-L_{x}+R_{z}\right)$ in $\operatorname{Ker} \Psi_{\mathbb{H}}$, where $x, y, z \in \operatorname{Im} \mathbb{H}$, and consider it as an element of $\wedge^{2} \mathbb{O}_{1} \oplus \wedge^{2} \mathbb{O}_{2} \oplus \wedge^{2} \mathbb{O}_{3}$, which we denote by $\left(\left(L_{x}+R_{y}, 0\right),\left(L_{z}-R_{y}, 0\right),\left(-L_{x}+R_{z}, 0\right)\right)$. Here $\left(L_{z}, 0\right)$, for example, denotes the endomorphism of $\mathbb{O}$ defined on elements of $\mathbb{H}$ by left multiplication by $z \in \mathbb{H}$, and by zero on $\mathbb{H}^{\perp}$. Note that with these conventions, it follows from the multiplication rules in the Cayley-Dickson process that for $z \in \operatorname{Im} \mathbb{H}$, we have $L_{z}=\left(L_{z},-L_{z}\right)$ and $R_{z}=\left(R_{z}, L_{z}\right)$.

It is easy to check that every skew-symmetric endomorphism of $\mathbb{H}$ is of the form $L_{a}+R_{b}$. In matrix terms, if $a=\alpha_{1} i+\alpha_{2} j+\alpha_{3} k$ and $b=\beta_{1} i+\beta_{2} j+\beta_{3} k$, we have

$$
L_{a}=\left(\begin{array}{cccc}
0 & -\alpha_{1} & -\alpha_{2} & -\alpha_{3} \\
\alpha_{1} & 0 & -\alpha_{3} & \alpha_{2} \\
\alpha_{2} & \alpha_{3} & 0 & -\alpha_{1} \\
\alpha_{3} & -\alpha_{2} & \alpha_{1} & 0
\end{array}\right), \quad R_{b}=\left(\begin{array}{cccc}
0 & -\beta_{1} & -\beta_{2} & -\beta_{3} \\
\beta_{1} & 0 & \beta_{3} & \beta_{2} \\
\beta_{2}-\beta_{3} & 0 & \beta_{1} \\
\beta_{3} & \beta_{2} & -\beta_{1} & 0
\end{array}\right)
$$

Under the natural isomorphism $\mathfrak{s o}(\mathbb{H}) \simeq \wedge^{2} \mathbb{H}$ induced by the norm on $\mathbb{H}$, this means that

$$
\begin{aligned}
& L_{a}=\alpha_{1}(1 \wedge i+j \wedge k)+\alpha_{2}(1 \wedge j+k \wedge i)+\alpha_{3}(1 \wedge k+i \wedge j), \\
& R_{b}=\beta_{1}(1 \wedge i-j \wedge k)+\beta_{2}(1 \wedge j-k \wedge i)+\beta_{3}(1 \wedge k-i \wedge j) .
\end{aligned}
$$

Now we can ask what is the element of $\mathfrak{t}(\mathbb{O})$ defined by $u_{1}=1 \wedge i \in \mathfrak{s o}(\mathbb{H}) \subset$ $\mathfrak{s o}(\mathbb{O})$ ? To answer this question, we just need to note that $1 \wedge i=\frac{1}{2}\left(L_{i}+R_{i}\right)$, so $u_{2}=\frac{1}{2} L_{i}$ and $u_{3}=\frac{1}{2} R_{i}$. Also if we take $u_{1}=j \wedge k$, a straightforward computation gives $u_{2}=j \wedge k+\frac{1}{2} R_{i}=\frac{1}{2}\left(L_{i}, L_{i}\right)$ and $u_{3}=j \wedge k-\frac{1}{2} L_{i}=\frac{1}{2}\left(-R_{i}, L_{i}\right)$. Letting automorphisms of $\mathbb{H}$ act, we deduce that the following triplets $\left(u_{1}, u_{2}, u_{3}\right)$ belong to $\mathfrak{t}(\mathbb{O})$, where $z, u, v$ denote imaginary quaternions:

$$
\begin{array}{lccccc}
u_{1} & 1 \wedge z & u \wedge v & \left(L_{z}, 0\right) & \left(R_{z}, 0\right) & \left(0, L_{z}\right) \\
u_{2} & \frac{1}{2} L_{z} & u \wedge v+\frac{1}{4} R_{[u, v]} & \left(L_{z}, 0\right) & \left(0,-L_{z}\right) & \left(R_{z}, 0\right) \\
u_{3} & \frac{1}{2} R_{z} & u \wedge v-\frac{1}{4} L_{[u, v]} & \left(0, L_{z}\right) & \left(R_{z}, 0\right) & \left(-L_{z}, 0\right)
\end{array}
$$


Thus we have

$$
\begin{aligned}
& \Psi_{\mathbb{O}}\left(\left(L_{x}, 0\right), 0,0\right)=\left(\left(L_{x}, 0\right),\left(0,-L_{x}\right),\left(L_{x}, 0\right)\right)=\Psi_{\mathbb{O}}\left(0,0,\left(L_{x}, 0\right)\right), \\
& \Psi_{\mathbb{O}}\left(\left(R_{y}, 0\right), 0,0\right)=\left(\left(R_{y}, 0\right),\left(R_{y}, 0\right),\left(0, L_{y}\right)\right)=\Psi_{\mathbb{\mathbb { N }}}\left(0,\left(R_{y}, 0\right), 0\right), \\
& \Psi_{\mathbb{O}}\left(0,\left(L_{z}, 0\right), 0\right)=\left(\left(0,-L_{z}\right),\left(L_{z}, 0\right),\left(-R_{z}, 0\right)\right)=\Psi_{\mathbb{O}}\left(0,0,\left(-R_{z}, 0\right)\right) .
\end{aligned}
$$

This immediately implies that $\left(\left(L_{x}+R_{y}, 0\right),\left(L_{z}-R_{y}, 0\right),\left(-L_{x}+R_{z}, 0\right)\right)$ belongs to $\operatorname{Ker} \Psi_{\mathbb{O}}$, and we deduce from the preceding formulas that the induced imbedding of $\mathfrak{t}(\mathbb{H}) \subset \mathfrak{t}(\mathbb{O})$ is given by

$$
\begin{aligned}
\left(L_{a}+R_{b}, L_{a}-R_{c},\right. & \left.L_{c}+R_{b}\right) \in \mathfrak{t}(\mathbb{H}) \\
& \mapsto\left(\left(L_{a}+R_{b}, L_{c}\right),\left(L_{a}-R_{c},-L_{b}\right),\left(L_{c}+R_{b}, L_{a}\right)\right) \in \mathfrak{t}(\mathbb{O}) .
\end{aligned}
$$

In particular, one can check that as a subalgebra of $\mathfrak{t}(\mathbb{O}), \mathfrak{t}(\mathbb{C})$ is generated by $\left(L_{i}+R_{i}, L_{i}, R_{i}\right)$ and $\left(R_{i},-R_{i}, L_{i}+R_{i}\right)$.

A more careful inspection of these formulas yields the explicit conclusion stated in the theorem.

\subsection{Automorphisms, Symmetric and Trisymmetric Spaces}

The symmetry in the triality model in the roles of $\mathbb{A}$ and $\mathbb{B}$ allows one to exhibit automorphisms of exceptional Lie algebras with interesting properties. For example:

Proposition 3.4. [62] The endomorphism of $\mathfrak{g}(\mathbb{A}, \mathbb{B})$ defined as the identity on $\mathfrak{h}(\mathbb{A}, \mathbb{B})=\mathfrak{t}(\mathbb{A}) \times \mathfrak{t}(\mathbb{B}) \oplus \mathbb{A}_{1} \otimes \mathbb{B}_{1}$, and minus the identity on $\mathbb{A}_{2} \otimes \mathbb{B}_{2} \oplus$ $\mathbb{A}_{3} \otimes \mathbb{B}_{3}$, is a Lie algebra involution.

Exhibiting a Lie algebra involution or a symmetric space is more or less equivalent, and we conclude that there exists a "magic square" of symmetric spaces of dimensions $2 a b$ where $a$ and $b$ denote the dimensions of $\mathbb{A}$ and $\mathbb{B}$. In particular we always get powers of 2 ! These are not all uniquely defined by our Lie algebra involution, but a version of this magic square is the following:

\begin{tabular}{|c|cccc|}
\hline & $\mathbb{R}$ & $\mathbb{C}$ & $\mathbb{H}$ & $\mathbb{O}$ \\
\hline $\mathbb{R}$ & $\mathbb{R} \mathbb{P}^{2}$ & $\mathbb{C P}^{2}$ & $\mathbb{H}^{2}$ & $\mathbb{O P}^{2}$ \\
$\mathbb{C}$ & $\mathbb{C P}^{2}$ & $\mathbb{C P}^{2} \times \mathbb{C P}^{2}$ & $G_{\mathbb{C}}(2,6)$ & $\mathbb{O P}_{\mathbb{C}}^{2}$ \\
$\mathbb{H}$ & $\mathbb{H}^{2}$ & $G_{\mathbb{C}}(2,6)$ & $G_{\mathbb{R}}(4,12)$ & $E_{7(-5)}$ \\
$\mathbb{O}$ & $\mathbb{O P}^{2}$ & $\mathbb{O} \mathbb{P}_{\mathbb{C}}^{2}$ & $E_{7(-5)}$ & $E_{8(8)}$ \\
\hline
\end{tabular}

The symmetric space $E_{8(8)}=E_{8} / \mathrm{SO}_{16}$, of dimension 128 , is particularly intriguing and it would be very nice to have a direct construction of it. It is claimed in [76] that it can be interpreted as a projective plane over $\mathbb{O} \otimes \mathbb{O}$, and that in fact the whole square above of symmetric spaces can be obtained by taking projective planes, in a suitable sense, over the tensor products $\mathbb{A} \otimes \mathbb{B}$.

It is also easy to describe Lie algebra automorphisms of order three, reflecting the trialitarian origin of the magic square. Recall that $\mathfrak{t}(\mathbb{A})$ has a natural automorphism $\tau_{\mathbb{A}}$ or order three. 
Proposition 3.5. The endomorphism of $\mathfrak{g}(\mathbb{A}, \mathbb{B})$ defined by

$$
\tau_{\mathbb{A}, \mathbb{B}}\left(X+Y+\mathrm{U}_{1}+\mathrm{U}_{2}+\mathrm{U}_{3}\right)=\tau_{\mathbb{A}}(X)+\tau_{\mathbb{B}}(Y)+\mathrm{U}_{2}+\mathrm{U}_{3}+\mathrm{U}_{1}
$$

for $X \in \mathfrak{t}(\mathbb{A}), Y \in \mathfrak{t}(\mathbb{B}), \mathrm{U}_{i} \in \mathbb{A}_{i} \otimes \mathbb{B}_{i}$, is a Lie algebra automorphism of order three, whose fixed points set is the subalgebra

$$
\mathfrak{k}(\mathbb{A}, \mathbb{B})=\operatorname{Der} \mathbb{A} \times \operatorname{Der} \mathbb{B} \oplus \mathbb{A} \otimes \mathbb{B} .
$$

When $\mathbb{B}=\mathbb{O}$, the conclusion also holds when we replace the usual triality automorphism $\tau_{\mathbb{O}}$ by its twisted version $\tau_{\mathbb{O}}^{\prime}$. In this case, the fixed point set is the subalgebra $\mathfrak{k}^{\prime}(\mathbb{A}, \mathbb{B})=\operatorname{Der} \mathbb{A} \times \mathfrak{s u} \mathfrak{u}_{3} \oplus \mathbb{A} \otimes \mathbb{B}$.

As in the case of involutions, we deduce from this statement a "magic square" of homogeneous spaces which are no longer symmetric, but are sometimes called trisymmetric: they are quotients of a semisimple Lie group $G$ by a subgroup $K$ which is, up to a finite group, the fixed point set of an automorphism of $G$ of order three. Their dimensions are equal to $2 a b+2 a+2 b-4$.

\begin{tabular}{|c|cccc|}
\hline & $\mathbb{R}$ & $\mathbb{C}$ & $\mathbb{H}$ & $\mathbb{O}$ \\
\hline $\mathbb{R}$ & $\mathrm{U}_{2} / \mathrm{U}_{1} \times \mathrm{U}_{1}$ & $\mathrm{U}_{3} /\left(\mathrm{U}_{1}\right)^{3}$ & $\mathrm{Sp}_{6} / \mathrm{U}_{2} \times \mathrm{Sp}_{2}$ & $F_{4} /\left(\mathrm{Spin}_{7} \times \mathrm{S}^{1} / \mathbb{Z}_{2}\right)$ \\
$\mathbb{C}$ & $\mathrm{U}_{3} /\left(\mathrm{U}_{1}\right)^{3}$ & $\mathrm{U}_{3} \times \mathrm{U}_{3} /\left(\mathrm{U}_{1}\right)^{6}$ & $\mathrm{U}_{6} /\left(\mathrm{U}_{2}\right)^{3}$ & $E_{6} / \mathrm{SO}_{8} \times\left(\mathrm{SO}_{2}\right)^{2}$ \\
$\mathbb{H}$ & $\mathrm{Sp}_{6} / \mathrm{U}_{2} \times \mathrm{Sp}_{2}$ & $\mathrm{U}_{6} /\left(\mathrm{U}_{2}\right)^{3}$ & $\mathrm{SO}_{12} / \mathrm{U}_{4} \times \mathrm{SO}_{4}$ & $E_{7} / S\left(\mathrm{U}_{7} \times \mathrm{U}_{1}\right) / \mathbb{Z}_{2}$ \\
$\mathbb{O}$ & $F_{4} /\left(\mathrm{Spin}_{7} \times \mathrm{S}^{1} / \mathbb{Z}_{2}\right)$ & $E_{6} / \mathrm{SO}_{8} \times\left(\mathrm{SO}_{2}\right)^{2}$ & $E_{7} / \mathrm{S}_{\left(\mathrm{U}_{7} \times \mathrm{U}_{1}\right) / \mathbb{Z}_{2}}$ & $E_{8} / \mathrm{SO}_{14} \times \mathrm{SO}_{2}$ \\
\hline
\end{tabular}

Here we denoted by $F_{4}, E_{6}, E_{7}, E_{8}$ the compact centerless groups of these types. Note that for example, $\mathrm{U}_{3} / \mathrm{U}_{1} \times \mathrm{U}_{1} \times \mathrm{U}_{1}$ is the variety of complete flags in $\mathbb{C}^{3}$. Similarly, $\mathrm{U}_{6} / \mathrm{U}_{2} \times \mathrm{U}_{2} \times \mathrm{U}_{2}$ is the flag variety $\mathbb{F}(2,4,6)$. Also $\mathrm{SO}_{12} / \mathrm{U}_{4} \times \mathrm{SO}_{4}$ is the space of 8 -dimensional subspaces in $\mathbb{R}^{12}$ endowed with an orthogonal complex structure. This is because $\mathrm{SO}_{2 n}$ acts transitively on the space of orthogonal complex structures. Moreover, the stabilizer of a point is the group of orthogonal transformations commuting with the corresponding complex structure, and this subgroup of $\mathrm{SO}_{2 n}$ is a copy of $\mathrm{U}_{n}$.

In the twisted case, for $\mathbb{B}=\mathbb{O}$, we replace the last line by

$$
F_{4} /\left(\mathrm{SU}_{3} \times \mathrm{SU}_{3} / \mathbb{Z}_{3}\right) \quad E_{6} /\left(\mathrm{SU}_{3} \times \mathrm{SU}_{3} \times \mathrm{SU}_{3} / \mathbb{Z}_{3}\right) \quad E_{7} /\left(\mathrm{SU}_{3} \times \mathrm{SU}_{6} / \mathbb{Z}_{3}\right) \quad E_{8} / \mathrm{SU}_{3} \times E_{6},
$$

a series of trisymmetric spaces of dimension $18 a+18$. Note that at the infinitesimal level, we obtain the quotients $\mathfrak{g}(\mathbb{A}, \mathbb{O}) / \mathfrak{s u}_{3} \times \mathfrak{g}(\mathbb{A}, \mathbb{C})$.

Automorphisms of order three were studied and classified by Wolf and Gray [34]. In the exceptional cases the construction above is not far from giving the whole classification.

\subsection{Dual Pairs}

The triality model allows one to identify series of dual pairs in the exceptional Lie algebras. Recall that a pair $\left(\mathfrak{h}, \mathfrak{h}^{\prime}\right)$ of Lie subalgebras of a Lie algebra $\mathfrak{g}$ is a dual pair if $\mathfrak{h}$ is the centralizer of $\mathfrak{h}^{\prime}$ and vice versa. Dual pairs of Lie 
algebras, or of Lie groups (with the same definition), have been extensively studied after the discovery by R. Howe that certain dual pairs of groups inside the real symplectic group $\operatorname{Sp}(2 n, \mathbb{R})$ have very special properties with respect to the (infinite dimensional) metaplectic representation [43].

First recall that, as we saw in 4.7 , the triality model is compatible with inclusions, in the sense that $\mathfrak{g}(\mathbb{A}, \mathbb{B})$ is naturally embedded in $\mathfrak{g}\left(\mathbb{A}^{\prime}, \mathbb{B}^{\prime}\right)$ when $\mathbb{A} \subset \mathbb{A}^{\prime}$ and $\mathbb{B} \subset \mathbb{B}^{\prime}$.

Proposition 3.6. The centralizers in $\mathfrak{g}(\mathbb{A}, \mathbb{O})$ of the subalgebras $\mathfrak{g}(\mathbb{A}, \mathbb{H})$, $\mathfrak{g}(\mathbb{A}, \mathbb{C}), \mathfrak{g}(\mathbb{A}, \mathbb{R})$ and $\mathfrak{t}(\mathbb{A})$, are isomorphic to $\mathfrak{s u}_{2}, \mathfrak{s u}_{3}, \mathfrak{g}_{2}$ and $\mathfrak{z}(\mathfrak{t}(\mathbb{A})) \times \mathfrak{s o}_{8}$, respectively. Moreover, the centralizers of these centralizers are the subalgebras themselves.

Here we denoted by $\mathfrak{z}(\mathfrak{t}(\mathbb{A}))$ the center of $\mathfrak{t}(\mathbb{A})$, which is $\mathfrak{t}(\mathbb{A})$ itself when this algebra is commutative, that is for $\mathbb{A}=\mathbb{R}$ or $\mathbb{C}$, and zero otherwise.

Proof. It is easy to see that the centralizer of $\mathfrak{g}(\mathbb{A}, \mathbb{B})$ inside $\mathfrak{g}(\mathbb{A}, \mathbb{O})$ is a subalgebra of $\mathfrak{t}(\mathbb{O})$, equal to the subalgebra of the centralizer of $\mathfrak{t}(\mathbb{B})$ inside $\mathfrak{t}(\mathbb{O})$ acting trivially on each $\mathbb{B}_{i} \subset \mathbb{O}_{i}$.

If $\mathbb{B}=\mathbb{R}$ we get the subalgebra of elements $\left(u_{1}, u_{2}, u_{3}\right) \in \mathfrak{t}(\mathbb{O})$ killing the unit element in each $\mathbb{O}_{i}$. Making $x=1$ and $y=1$ in the triality relation $u_{1}(x y)=u_{2}(x) y+x u_{3}(y)$, we see that $u_{1}=u_{2}=u_{3}$ is a derivation of $\mathbb{O}$, so that the center of $\mathfrak{g}(\mathbb{A}, \mathbb{R})$ inside $\mathfrak{g}(\mathbb{A}, \mathbb{O})$ is Der $\mathbb{O}=\mathfrak{g}_{2}$.

When $\mathbb{B}=\mathbb{C}$, the condition that $\left(u_{1}, u_{2}, u_{2}\right)$ kills $\mathbb{C}_{i} \subset \mathbb{O}_{i}$ implies that $u=$ $u_{1}=u_{2}=u_{3}$ is a derivation of $\mathbb{O}$ such that $u(i)=0\left(i=e_{1}\right.$ in the notations of 5.1). But then $u(i x)=i u(x)$, which means that $u$ is a complex endomorphism of $\mathbb{C}^{\perp} \simeq \mathbb{C}^{3}$, endowed with the complex structure defined by $L_{i}$ (and also that defined by $R_{i}$, which is the conjugate complex structure, since $u(x i)=$ $u(x) i)$. Note that the corresponding element of $\mathfrak{t}(\mathbb{O})$ automatically centralizes $\mathfrak{t}(\mathbb{C})$, since $u$ commutes with $L_{i}, R_{i}$ and we saw that $\mathfrak{t}(\mathbb{C})$ is generated as a subalgebra of $\mathfrak{t}(\mathbb{O})$ by $\left(L_{i}+R_{i}, L_{i}, R_{i}\right)$ and $\left(R_{i},-R_{i}, L_{i}+R_{i}\right)$. Finally, take the general matrix of $\mathfrak{g}_{2}$ as written in Section 3.2. The condition that $u(i)=0$ means that $\alpha_{2}=\alpha_{3}=\alpha_{4}=\alpha_{5}=\alpha_{6}=\alpha_{7}=0$. A complex basis of the space $\mathbb{C}^{\perp}$ is $\left(e_{2}, e_{4}, e_{6}\right)$, in terms of which $u$ is the complex endomorphism given by the matrix

$$
\left(\begin{array}{ccc}
i \beta_{3} & -\beta_{4}+i \beta_{5} & -\beta_{6}+i \beta_{7} \\
\beta_{4}+i \beta_{5} & i \gamma_{5} & -\gamma_{6}+i \gamma_{7} \\
\beta_{6}+i \beta_{7} & \gamma_{6}+i \gamma_{7} & -i\left(\beta_{3}+\gamma_{5}\right)
\end{array}\right),
$$

which is a general element of $\mathfrak{s u}_{3}$.

Finally let $\mathbb{B}=\mathbb{H}$. Again, our description of $\mathfrak{g}_{2}$ in Section 3.2 shows that the derivations of $\mathbb{O}$ vanishing on $\mathbb{H}$ form a copy of $\mathfrak{s o}_{3}$, which we can describe as the space of endomorphisms of the form $\left(0, R_{z}\right)$ with $z \in \operatorname{ImHH}$. But we saw that $\mathfrak{t}(\mathbb{H})$, as a subalgebra of $\mathfrak{t}(\mathbb{C})$, was generated by the triplets $\left(\left(L_{a}+\right.\right.$ $\left.\left.R_{b}, L_{c}\right),\left(L_{a}-R_{c},-L_{b}\right),\left(L_{c}+R_{b}, L_{a}\right)\right)$, where $a, b, c \in \operatorname{Im} \mathbb{H}$. But these elements of $\mathfrak{t}(\mathbb{O})$ commute with those of the form $\left(\left(0, R_{z}\right),\left(0, R_{z}\right),\left(0, R_{z}\right)\right)$. This proves that the centralizer of $\mathfrak{g}(\mathbb{A}, \mathbb{H})$ in $\mathfrak{g}(\mathbb{A}, \mathbb{O})$ is $\mathfrak{s o}_{3}$. 
Alternatively we can identify this centralizer to the subalgebra of the centralizer of $\mathfrak{t}(\mathbb{C})$ annihilating $e_{2}=j$, which is clearly $\mathfrak{s u}_{2}$.

The rest of the claim is straightforward.

In particular, we have pairs of reductive subalgebras in $\mathfrak{g}(\mathbb{A}, \mathbb{O})$, each of which is the centralizer of the other: this is a reductive dual pair. The classification of reductive dual pairs inside reductive complex Lie algebras was obtained in [77]. We deduce from the proposition above the existence of uniform series of what Rubenthaler calls towers of reductive dual pairs:

\begin{tabular}{|c|c|}
\hline $\mathfrak{g}(\mathbb{A}, \mathbb{O})$ & $\mathfrak{g}(\mathbb{A}, \mathbb{O})$ \\
\hline$\cup$ & $U$ \\
\hline $\mathfrak{g}(\mathbb{A}, \mathbb{H})$ & $\mathfrak{s o}_{8} \times \mathfrak{z}(\mathfrak{t}(\mathbb{A}))$ \\
\hline$U$ & $U$ \\
\hline $\mathfrak{g}(\mathbb{A}, \mathbb{C})$ & $\mathfrak{g}_{2}$ \\
\hline$U$ & $U$ \\
\hline $\mathfrak{g}(\mathbb{A}, \mathbb{R})$ & $\mathfrak{s u}_{3}$ \\
\hline$U$ & $U$ \\
\hline $\mathfrak{t}(\mathbb{A})$ & $\mathfrak{s u}_{2}$ \\
\hline
\end{tabular}

We conclude that $\mathfrak{s o}_{8} \times \mathfrak{t}(\mathbb{A}), \mathfrak{g}_{2} \times \mathfrak{g}(\mathbb{A}, \mathbb{R}), \mathfrak{s u}_{3} \times \mathfrak{g}(\mathbb{A}, \mathbb{C})$ and $\mathfrak{s o}_{3} \times \mathfrak{g}(\mathbb{A}, \mathbb{H})$ are maximal rank reductive subalgebras of $\mathfrak{g}(\mathbb{A}, \mathbb{O})$. A natural question to ask is to describe the module structure of $\mathfrak{g}(\mathbb{A}, \mathbb{O})$ over each of these subalgebras. For the first one, the answer is given by the triality construction. For the second one, we note that $\mathfrak{g}(\mathbb{A}, \mathbb{R})=\operatorname{Der}\left(\mathcal{J}_{3}(\mathbb{A})\right)$ and $\mathfrak{g}_{2}=\operatorname{Der} \mathbb{O}$, so that we are back to the original Tits construction of the exceptional series of Lie algebras. We obtain:

$$
\begin{aligned}
\mathfrak{g}(\mathbb{A}, \mathbb{O}) & =\mathfrak{g}_{2} \times \mathfrak{g}(\mathbb{A}, \mathbb{R}) \oplus \mathbb{R}^{7} \otimes \mathcal{J}_{3}(\mathbb{A})_{0} \\
& =\mathfrak{s u}_{3} \times \mathfrak{g}(\mathbb{A}, \mathbb{C}) \oplus \mathbb{R}^{3} \otimes \mathcal{J}_{3}(\mathbb{A}) \oplus\left(\mathbb{R}^{3} \otimes \mathcal{J}_{3}(\mathbb{A})\right)^{*} \\
& =\mathfrak{s u}_{2} \times \mathfrak{g}(\mathbb{A}, \mathbb{H}) \oplus \mathbb{R}^{2} \otimes \mathcal{Z}_{3}(\mathbb{A})
\end{aligned}
$$

Here $\mathcal{Z}_{3}(\mathbb{A})$ denotes the space of Zorn matrices, which is a $\mathfrak{g}(\mathbb{A}, \mathbb{H})$-module, see Section 4.6. Note that $\mathfrak{s u}_{2}$ acts not on $\mathbb{R}^{2}$ but on $\mathbb{C}^{2}$. Therefore there must exist a complex structure on $\mathcal{Z}_{3}(\mathbb{A})=\mathcal{Z}_{3}^{0}(\mathbb{A}) \oplus i \mathcal{Z}_{3}^{0}(\mathbb{A})$, which is not $\mathfrak{g}(\mathbb{A}, \mathbb{H})$-invariant, so that $\mathbb{R}^{2} \otimes \mathcal{Z}_{3}(\mathbb{A})=\mathbb{C}^{2} \otimes \mathcal{Z}_{3}^{0}(\mathbb{A})$, the $\mathfrak{s u}_{2}$-action coming from $\mathbb{C}^{2}$.

These towers of dual pairs in series were discovered in joint work with B. Westbury.

\subsection{The Quaternionic Form}

The algebras $\mathfrak{g}(\mathbb{A}, \tilde{\mathbb{B}})$ are also interesting, especially when $\mathbb{B}=\mathbb{O}$. Following Wolf [84], each simple complex Lie algebra has a unique real form such that the associated compact or non-compact symmetric spaces have an invariant quaternionic structure. We will say that this real form is quaternionic. 
Recall that the rank of a symmetric space $G / K$, corresponding to some Cartan decomposition $\mathfrak{g}=\mathfrak{k} \oplus \mathfrak{p}$, is the maximal dimension of a subalgebra of $\mathfrak{g}$ contained in $\mathfrak{p}$. Such a subalgebra is automatically abelian and is called a Cartan subspace.

Theorem 3.7. The real Lie algebra $\mathfrak{g}(\mathbb{A}, \tilde{\mathbb{O}})$ is quaternionic. A Cartan subspace of $\mathfrak{t}(\tilde{\mathbb{O}})=\mathfrak{s o}_{4,4}$ embeds as a Cartan subspace of $\mathfrak{g}(\mathbb{A}, \tilde{\mathbb{O}})$, which in particular has real rank four (independent of $\mathbb{A}$ ).

Proof. Recall that each simple complex Lie algebra $\mathfrak{g}$, once a Cartan subalgebra and a set of positive roots has been chosen, has a canonical 5-graduation defined by the highest root $\tilde{\alpha}$, more precisely by the eigenspaces of $\operatorname{ad}\left(H_{\tilde{\alpha}}\right)$, where $H_{\tilde{\alpha}}$ denotes the coroot of $\alpha$.

If $\mathfrak{g}$ is the complexification of the compact real Lie algebra $\mathfrak{g}(\mathbb{A}, \mathbb{O})$, we can arrange so that the highest root comes from $\mathfrak{t}(\mathbb{O})_{\mathbb{C}}=\mathfrak{s o}_{8}(\mathbb{C})$. Note that the space of highest root vectors in $\mathfrak{s o}_{8}(\mathbb{C})$ is the cone over the Grassmannian of isotropic planes in $\mathbb{C}^{8}$. Using this, we then check that we can arrange so that

$$
i H_{\tilde{\alpha}}=\left(\begin{array}{cccccccc}
0 & 0 & 0 & 0 & 0 & 0 & 0 & 0 \\
0 & 0 & 0 & 0 & 0 & 0 & 0 & 0 \\
0 & 0 & 0 & 0 & 0 & 0 & 0 & 0 \\
0 & 0 & 0 & 0 & 0 & 0 & 0 & 0 \\
0 & 0 & 0 & 0 & 0 & -1 & 0 & 0 \\
0 & 0 & 0 & 0 & 1 & 0 & 0 & 0 \\
0 & 0 & 0 & 0 & 0 & 0 & 0 & -1 \\
0 & 0 & 0 & 0 & 0 & 0 & 1 & 0
\end{array}\right) .
$$

The eigenvalues of $\operatorname{ad}\left(H_{\tilde{\alpha}}\right)$ acting on $\mathfrak{g}$ are $0, \pm 1, \pm 2$. The corresponding eigenspaces are

$$
\begin{aligned}
\mathfrak{g}_{0} & =\mathfrak{t}\left(\mathbb{A}_{\mathbb{C}}\right) \times \mathfrak{t}\left(\mathbb{O}_{\mathbb{C}}\right)_{0} \oplus \mathbb{A}_{\mathbb{C}, 1} \otimes \mathbb{O}_{\mathbb{C}, 1}^{0} \oplus \mathbb{A}_{\mathbb{C}, 2} \otimes \mathbb{O}_{\mathbb{C}, 2}^{0} \oplus \mathbb{A}_{\mathbb{C}, 3} \otimes \mathbb{O}_{\mathbb{C}, 3}^{0}, \\
\mathfrak{g}_{ \pm 1} & =\mathfrak{t}\left(\mathbb{O}_{\mathbb{C}}\right)_{ \pm 1} \oplus \mathbb{A}_{\mathbb{C}, 1} \otimes \mathbb{O}_{\mathbb{C}, 1}^{ \pm 1} \oplus \mathbb{A}_{\mathbb{C}, 2} \otimes \mathbb{O}_{\mathbb{C}, 2}^{ \pm 1} \oplus \mathbb{A}_{\mathbb{C}, 3} \otimes \mathbb{O}_{\mathbb{C}, 3}^{ \pm 1} \\
\mathfrak{g}_{ \pm 2} & =\mathfrak{t}\left(\mathbb{O}_{\mathbb{C}}\right)_{ \pm 2}
\end{aligned}
$$

where we denoted by $\mathbb{O}_{i}^{t}$ the $t$-eigenspace for the action of $H_{\tilde{\alpha}}$ on $\mathbb{O}_{i}$. Note that $i H_{\tilde{\alpha}}$ belongs to Der $\mathbb{O}=\mathfrak{g}_{2}$ (see our description of $\mathfrak{g}_{2}$ above), so we can let $i=1$ and consider the standard action of $H_{\tilde{\alpha}}$. Then it is clear that the kernel of $H_{\tilde{\alpha}}$ is the complexification of the standard quaternion subalgebra $\mathbb{H}$, and that the sum of the two other eigenspaces is the complexification of $e \mathbb{H}$.

Now, let $\theta$ denote the involution of $\mathfrak{g}$ associated to its 5 -graduation. By definition, $\theta$ acts by $(-1)^{k}$ on $\mathfrak{g}_{k}$. Then $\theta$ stabilizes $\mathfrak{g}(\mathbb{A}, \mathbb{O})$, and the corresponding Cartan decomposition $\mathfrak{g}(\mathbb{A}, \mathbb{O})=\mathfrak{k} \oplus \mathfrak{p}$ into eigenspaces of $\theta$ is given, in terms of the Cartan decomposition $\mathfrak{t}(\mathbb{O})=\mathfrak{k}_{\mathbb{O}} \oplus \mathfrak{p}_{\mathbb{O}}$, by

$$
\begin{aligned}
& \mathfrak{k}=\mathfrak{t}(\mathbb{A}) \times \mathfrak{k}_{\mathbb{O}} \oplus \mathbb{A}_{1} \otimes \mathbb{H}_{1} \oplus \mathbb{A}_{2} \otimes \mathbb{H}_{2} \oplus \mathbb{A}_{3} \otimes \mathbb{H}_{3} \\
& \mathfrak{p}=\mathfrak{p}_{\mathbb{O}} \oplus \mathbb{A}_{1} \otimes e \mathbb{H}_{1} \oplus \mathbb{A}_{2} \otimes e \mathbb{H}_{2} \oplus \mathbb{A}_{3} \otimes e \mathbb{H}_{3}
\end{aligned}
$$


Finally (see $[84,17]$ ), we obtain the quaternionic form $\mathfrak{g}(\mathbb{A}, \mathbb{O})_{\mathbb{H}}$ of $\mathfrak{g}$ by twisting this decomposition, that is, letting $\mathfrak{g}(\mathbb{A}, \mathbb{O})_{\mathbb{H}}=\mathfrak{k} \oplus i \mathfrak{p}$, which amounts to multiplying the brackets in $\mathfrak{g}(\mathbb{A}, \mathbb{O})$ of two elements of $\mathfrak{p}$ by -1 . From the description of $\mathfrak{k}$ and $\mathfrak{p}$ we have just given, we see that this twist amounts to doing two things. First, twist the Cartan decomposition of $\mathfrak{t}(\mathbb{O})$, which means that we replace $\mathfrak{t}(\mathbb{O})=\mathfrak{s o}_{8}$ by its quaternionic form $\mathfrak{s o}_{4,4}=\mathfrak{t}(\tilde{\mathbb{O}})$. Second, twist the Cayley-Dickson process of construction of $\mathbb{O}$ from $\mathbb{H}$ by multiplying by -1 the product of two elements in $e \mathbb{H}$, which amounts to replace $\mathbb{O}$ by the split Cayley algebra $\tilde{\mathbb{O}}$. This proves the first part of the theorem. The rest of the proof is straightforward.

\section{Adjoint Varieties and Quaternionic Symmetric Spaces}

Symmetric spaces with quaternionic structures are closely related to adjoint varieties. Recall that on a Riemannian manifold $M$, a quaternionic structure is defined as a parallel field of quaternion algebras $\mathbb{H}_{x} \subset \operatorname{End}\left(T_{x} M\right), x \in M$, such that the unit sphere of $\mathbb{H}_{x}$ is contained in the orthogonal group $\mathrm{SO}\left(T_{x} M\right)$. The dimension of $M$ is then equal to $4 m$ for some $m>0$, and its reduced holonomy group is contained in $\operatorname{Sp}(m) \operatorname{Sp}(1) \subset \mathrm{SO}(4 m)$, where $\operatorname{Sp}(m)$ denotes the group of quaternionic unitary matrices of order $m$ (in particular, $\operatorname{Sp}(1) \simeq S^{3}$ is the unit sphere in $\mathbb{H}$ ). Such matrices act on $\mathbb{H}^{m}=\mathbb{R}^{4 m}$ by multiplication on the left, which commutes with the scalar multiplication on the right by unitary quaternions: the resulting group of orthogonal transformations of $\mathbb{R}^{4 m}$ is $\operatorname{Sp}(m) \operatorname{Sp}(1)$.

This group appears in Berger's classification as one of the few possible reduced holonomy groups of nonsymmetric Riemannian manifolds. The case of symmetric manifolds was discussed in detail by J. Wolf in [84], who proved that there exists exactly one $G$-homogeneous symmetric space $M$ with a quaternionic structure for each simple compact Lie group $G$. When $G$ is simply connected, we can write $M=G / K . \operatorname{Sp}(1)$. Choosing a complex plane in $\mathbb{H}$ amounts to choosing a circle $\mathrm{S}^{1} \subset \mathrm{Sp}(1)$, and this induces a fibration

$$
X=G / K . \mathrm{S}^{1} \longrightarrow M=G / K \cdot \operatorname{Sp}(1)
$$

whose fibers are two-spheres. Wolf proved that $X$ is a complex variety, homogeneous under the complexification $G^{\mathbb{C}}$ of the real compact group $G$, and endowed with an invariant contact structure. Such varieties were previously classified by Boothby, who showed that they are in correspondance with simple complex Lie groups of adjoint type. In fact, $X$ is the adjoint variety $\left(G^{\mathbb{C}}\right)^{\text {ad }}$, the closed orbit in $\mathbb{P g}^{\mathbb{C}}$.

In modern terminology, $X$ is the twistor space of $M$, and Wolf's work is at the origin of this twistor theory, which assigns to each Riemannian manifold $M$ with a quaternionic structure a complex variety $X$ which is a $S^{2}$-bundle over $M$, defined at each point $x \in M$ by the unit sphere in $\operatorname{Im}_{\mathbb{H}_{x}}$. 
The adjoint varieties are endowed with natural contact structures (induced by the Kostant-Kirillov symplectic structures on the minimal nilpotent coadjoint orbits, and of which the adjoint varieties are the projectivizations). In fact, any compact Riemannian manifold with exceptional holonomy $\mathrm{Sp}(n) \operatorname{Sp}(1)$ and positive scalar curvature has a twistor space that is a contact Fano manifold. Our understanding of contact Fano manifolds has recently improved very much. First of all, there are not very many:

Theorem 3.8. (LeBrun-Salamon [69]) Up to biholomorphism, there are only finitely many contact Fano manifolds of any given dimension.

This lead to the following conjecture:

Conjecture. (LeBrun-Salamon) Let $X$ be a smooth complex projective contact variety with $b_{2}(X)=1$. Then $X$ must be the adjoint variety of a simple complex Lie group.

Both the theorem and conjecture can be rephrased purely on the Riemannian side using the twistor transform.

In [52], S. Kebekus proved that the space of minimal rational curves through a fixed point of $X$ is a Legendre variety (except for the case of $\mathbb{P}^{2 n-1}$ ), just as is the case for the adjoint varieties.

\section{Series Of Lie Algebras Via Knot Theory and Geometry}

\subsection{From Knot Theory To the Universal Lie Algebra}

One of the main achievements of the last ten years in topology has been the definition of the Kontsevich integral, which is a universal invariant of finite type for knots. The Kontsevich integral associates to each knot a formal series in a space of chord diagrams. The space is a quotient of the space of formal combinations of certain types of graphs by the AS and IHX relations. From the Kontsevitch integral, it is possible to deduce more standard invariants, say numerical or polynomial invariants, if one is given a quadratic Lie algebra, i.e., a finite dimensional Lie algebra endowed with an invariant nondegenerate quadratic form. An essential point is that the AS and IHX relations can be interpreted respectively as the antisymmetry of the Lie bracket and the Jacobi identity.

The main reference for the Kontsevich integral is the beautiful article by Bar-Natan [6]. In it he defines and proves (Theorem 4) the mapping from quadratic Lie algebras equipped with a representation to a functional on chord diagrams (called a weight system) and makes the conjecture (Conjecture 1) that all weight systems come from this mapping. This conjecture was disproved by P. Vogel in [82] which raised the problem of constructing a category 
more general than that of quadratic Lie algebras that would give rise to all weight systems.

In [83] Vogel defines a candidate for such an object, which he calls the universal Lie algebra. The universal Lie algebra is a category $\mathcal{D}$ with the following property: for any quadratic Lie (super)algebra $\mathfrak{g}$ over a field $k$, there is a natural functor from $\mathcal{D}$ to the category $\operatorname{Mod}_{k} \mathfrak{g}$ of $\mathfrak{g}$-modules. The objects of the category $\mathcal{D}$ are finite sets $[n]=\{1, \ldots, n\}, n \geq 0$. Morphisms in $\mathcal{D}$ are defined as follows: $\operatorname{Hom}_{\mathcal{D}}([p],[q])$ is the $\mathbb{Z}$-module of formal combinations of uni-trivalent abstract graphs with univalent vertices $[p] \cup[q]$, modulo isotopy, and modulo the AS and IHX relations. If $\mathfrak{g}$ is a quadratic Lie algebra, a functor from $\mathcal{D}$ to $\operatorname{Mod}_{k} \mathfrak{g}$ is defined at the level of objects by sending $[n]$ to $\mathfrak{g}^{\otimes n}$. To determine the morphisms, consider some uni-trivalent graph defining an element of $\operatorname{Hom}_{\mathcal{D}}([p],[q])$. Possibly after isotopy, such a graph can be seen as made of elementary pieces of the form

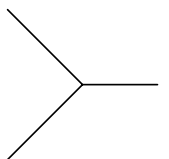

bracket

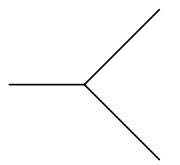

cobracket

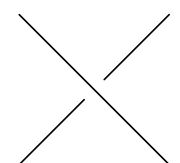

symmetry

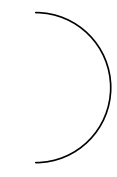

quadratic

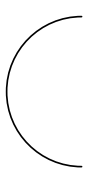

Casimir

These pieces should be interpreted as indicated, respectively as the Lie bracket $\mathfrak{g}^{\otimes 2} \rightarrow \mathfrak{g}$, the cobracket $\mathfrak{g} \rightarrow \mathfrak{g}^{\otimes 2}$ (the dual to the bracket), the symmetry $\mathfrak{g}^{\otimes 2} \rightarrow \mathfrak{g}^{\otimes 2}$, the quadratic form $\mathfrak{g}^{\otimes 2} \rightarrow \mathfrak{g}^{\otimes 0}=k$, and the Casimir element $\mathfrak{g}^{\otimes 0} \rightarrow \mathfrak{g}^{\otimes 2}$ (the dual to the quadratic form). Putting together the contributions of all its elementary pieces, one associates a morphism from $\mathfrak{g}{ }^{\otimes p}$ to $\mathfrak{g}^{\otimes q}$ to the graph. This morphism depends only on the isotopy class of the graph, and factors through the AS and IHX relations, because of the skew-symmetry and Jacobi relation for the Lie bracket in $\mathfrak{g}$.

Vogel proves a stronger result for simple quadratic Lie algebras. One can then replace the category $\mathcal{D}$ by another one $\mathcal{D}^{\prime}$, which is deduced from $\mathcal{D}$ by forcing an action of the algebra $\Lambda$ of skew-symmetric connected uni-trivalent graphs with exactly three univalent vertices. The functor from $\mathcal{D}^{\prime}$ to $\operatorname{Mod}_{k} \mathfrak{g}$ is then compatible with the action of $\Lambda$ through a character $\chi_{\mathfrak{g}}: \Lambda \rightarrow k$.

From the point of view of representation theory, the existence and the properties of the categories $\mathcal{D}$ and $\mathcal{D}^{\prime}$ have very interesting consequences. Indeed, each "decomposition" of $[p]$ in $\mathcal{D}^{\prime}$ will imply the existence of a decomposition of $\mathfrak{g} \otimes p$ of the same type, for every simple quadratic Lie algebra. Here, by a "decomposition" of $[p]$, we must understand a decomposition of the identity endomorphism of $[p]$ as a sum of idempotents, which will correspond to projectors onto submodules of $\mathfrak{g} \otimes p$.

Describing such idempotents is far from easy and requires quite subtle computations with uni-trivalent graphs. For $p=2$, Vogel obtained the following result. (Vogel's proof relied on conjecture 3.6 as stated in [83], but Vogel himself realized that it is not correct as $\Lambda$, which is conjectured to be integral, 
has zero divisors. Nevertheless, a case by case verification shows that Vogel's conclusions are indeed correct.)

Let $\mathfrak{g}$ be a simple Lie algebra, say over the complex numbers. Then there are decompositions

$$
\begin{aligned}
& \wedge^{2} \mathfrak{g}=X_{1} \oplus X_{2}, \\
& S^{2} \mathfrak{g}=X_{0} \oplus Y_{2} \oplus Y_{2}^{\prime} \oplus Y_{2}^{\prime \prime},
\end{aligned}
$$

into simple $\mathfrak{g}$-modules (some of which may be zero), with $X_{0}=\mathbb{C}$ and $X_{1}=$ $\mathfrak{g}$. Moreover, there exists $\alpha, \beta, \gamma$ such that one half the Casimir operator acts on $X_{1}, X_{2}, Y_{2}, Y_{2}^{\prime}$ and $Y_{2}^{\prime \prime}$ by multiplication by $t, 2 t, 2 t-\alpha, 2 t-\beta$ and $2 t-\gamma$ respectively, where $t=\alpha+\beta+\gamma$. (In fact the Casimir organizes the representations in series, e.g., the representations $X_{k}$ have Casimir eigenvalue $k t$.) Finally, the dimensions of these $\mathfrak{g}$-modules are given by rational functions in $\alpha, \beta, \gamma$ (the dimensions of $Y_{2}^{\prime}$ and $Y_{2}^{\prime \prime}$ are deduced from that of $Y_{2}$ by cyclic permutations of $\alpha, \beta, \gamma)$ :

$$
\begin{gathered}
\operatorname{dimg}=\frac{(\alpha-2 t)(\beta-2 t)(\gamma-2 t)}{\alpha \beta \gamma}, \\
\operatorname{dim} X_{2}=-\frac{(\alpha-2 t)(\beta-2 t)(\gamma-2 t)(\alpha+t)(\beta+t)(\gamma+t)}{\alpha^{2} \beta^{2} \gamma^{2}}, \\
\operatorname{dim} Y_{2}=-\frac{t(\beta-2 t)(\gamma-2 t)(\beta+t)(\gamma+t)(3 \alpha-2 t)}{\alpha^{2} \beta \gamma(\alpha-\beta)(\alpha-\gamma)}
\end{gathered}
$$

The scalars $\alpha, \beta, \gamma$ are readily computed for each simple complex Lie algebra. Note that they are defined only up to permutation and multiplication by a same scalar (this is because the Casimir operator has not been normalized). We can therefore consider $(\alpha, \beta, \gamma)$ as a point in $\mathbb{P}^{2} / \mathfrak{S}_{3}$.

$\begin{array}{cccll}\text { Series } & \text { Lie algebra } & \alpha & \beta & \gamma \\ \text { SL } & \mathfrak{s l}_{n} & -2 & 2 & n \\ \text { OSP } & \mathfrak{s o}_{n}, \mathfrak{s p}_{-n} & -2 & 4 & n-4 \\ \text { EXC } & \mathfrak{s l}_{3} & -2 & 3 & 2 \\ & \mathfrak{g}_{2} & -3 & 5 & 4 \\ & \mathfrak{s o}_{8} & -2 & 6 & 4 \\ & \mathfrak{f}_{4} & -2 & 5 & 6 \\ & \mathfrak{e}_{6} & -2 & 6 & 8 \\ & \mathfrak{e}_{7} & -2 & 8 & 12 \\ & \mathfrak{e}_{8} & -2 & 12 & 20\end{array}$

Note that up in $\mathbb{P}^{2}$, the points of the three series SL, OSP and EXC are located on three lines, respectively $\alpha+\beta=0,2 \alpha+\beta=0,2 \alpha+2 \beta-\gamma=0$. Hence the slogan that there exists only three complex simple Lie algebras, SL, OSP, and EXC. At the level of representations, it is a classical and well-known fact that the categories of modules over the $\mathfrak{s l}_{n}$, or the $\mathfrak{s o}_{n}$ and $\mathfrak{s p}_{2 n}$, have close and precise relationships. This is more surprising for the exceptional series. 
In Section 4.6 we discuss another collection of Lie algebras that form a "line" in Vogel's plane.

Remark 4.1. In a spirit similar to Vogel's, Cvitanovic [21] has proposed a proof of the Killing-Cartan classification. Using notation inspired by the Feynman diagrams of quantum field theory, he represents the invariant tensors of a Lie algebra by diagrams, and by requring that irreducible representations be of integer dimension, he deduces severe limits on what simple groups could possibly exist. His approach dates back to 1976 [19, 20]. The method provides the correct Killing-Cartan list of all possible simple Lie algebras, but fails to prove existence.

\subsection{Vogel's Decompositions and Tits Correspondences}

As remarked above, Tits correspondences aid one in decomposing the tensor powers of nice (e.g., fundamental) representations into irreducible factors. We illustrate this by showing how to recover Vogel's decompositions of $S^{2} \mathfrak{g}$ and $\Lambda^{2} \mathfrak{g}$. In fact we can recover Vogel's higher decompositions as well, but we need to use a slightly more general technique which we call diagram induction, see [63]. Using diagram induction, we are also able to recover the Casimir functions for the representations occuring in series such as the $X_{k}$.

If $V$ is a fundamental representation corresponding to a root that is not short (e.g., the fundamental adjoint representations) and $X=G / P_{\alpha} \subset \mathbb{P} V$ the closed orbit, then the Fano variety $\mathbb{F}_{1}(X)$ of $\mathbb{P}^{1}$ 's on $X$ is $G$-homogeneous by Theorem 2.5, and according to Tits fibrations, $\mathbb{F}_{1}(X)=G / P_{S}$ where $S$ is the set of roots joined to $\alpha$ in $D(\mathfrak{g})$. Let $V_{2}=\left\langle\mathbb{F}_{1}(X)\right\rangle$ denote the linear span of the cone over $\mathbb{F}_{1}(X)$ in its minimal homogeneous embedding. Note that $\mathbb{F}_{1}(X) \subset G(2, V) \subset \Lambda^{2} V$, and we conclude that $V_{2} \subset \Lambda^{2} V$. (To do this, one needs to make sure the embedding of $\mathbb{F}_{1}(X)$ is indeed the minimal one, which is one reason we need the stronger technique of diagram induction.)

In the case of the adjoint representation, we also know, because of the cobracket, that $\mathfrak{g} \subset \Lambda^{2} \mathfrak{g}$. In fact, we have $\Lambda^{2} \mathfrak{g}=\mathfrak{g} \oplus \mathfrak{g}_{2}$. (In the case of $\mathfrak{a}_{n}$, $\left(\mathfrak{a}_{n}\right)_{2}$ is the direct sum of two dual irreducible representations, which is also predicted by Tits fibrations.)

Similiarly, inside $S^{2} \mathfrak{g}$, we know there is a trivial representation $X_{0}$ corresponding to the Killing form, and the Cartan power $\mathfrak{g}^{(2)}$, which, breaking Vogel's symmetry, we set $Y_{2}=\mathfrak{g}^{(2)}$ so the problem becomes to recover the remaining modules, which we think of as the "primitive" ones. These are provided in most cases by Tits fibrations of homogeneous quadrics contained in $X$. There are up to two such families, we call the larger one $\mathfrak{g}_{Q}=Y_{2}^{\prime}$ and if there is a second we call it $\mathfrak{g}_{Q^{\prime}}=Y_{2}^{\prime \prime}$, see [63]. Thus, using Tit's correspondences to find the primitive factors, one recovers the Vogel decomposition of $\mathfrak{g}^{\otimes 2}$ for $\mathfrak{g}$ simple (except for $\mathfrak{c}_{n}$, which is tractible using diagram induction). Even when our methods predict all factors, we would like to emphasize that they do not explain why these are the only factors. 
While our observations enable one to recover uniform decompositions of plethysms in series, the questions of why such series exist and how to find them systematically without looking at the list of simple Lie algebras is mysterious using our present techniques. It would be wonderful and challenging to generalize our perspective sufficiently to approach Vogel's. One indication that such a generalization might be possible is that, with the above choices, we obtain a geometric interpretation of Vogel's parameter $\beta$, namely $\beta$ is the dimension of the largest quadric hypersurface contained in $X_{\mathrm{ad}}$ (see [63]). If there is a second unextendable quadric in $X_{\mathrm{ad}}$, then $\gamma$ is its dimension.

\subsection{The Exceptional Series}

Inspired by Vogel's work, Deligne [24], investigated the decompositions of the tensor powers of the exceptional Lie algebras into irreducible components, going up to degree four (with the help of Cohen, deMan and the computer program LiE, [14]) and giving more explicit dimension formulas.

\subsubsection{Decomposition Formulas}

As with the Vogel decompositions, these decomposition formulas are all uniform, in the sense, for example, that the numbers of irreducible components are always the same when the algebra varies in the exceptional series. This assertion has to be understood with some care: it happens that some components vanish, or should be taken with a minus sign. In addition, Deligne and Vogel deal with the algebra twisted by the symmetry of the marked (for the adjoint representation) diagram.

In degree two, one has the decomposition of Vogel, but the decomposition of $S^{2} \mathfrak{g}$ simplifies because the equation $2 \alpha+2 \beta-\gamma=0$ implies $Y_{2}^{\prime \prime}$ is zero. In degree three we have

$$
\begin{aligned}
S^{3} \mathfrak{g} & =\mathfrak{g} \oplus X_{2} \oplus A \oplus Y_{3} \oplus Y_{3}^{\prime}, \\
\wedge^{3} \mathfrak{g} & =\mathbb{C} \oplus X_{2} \oplus Y_{2} \oplus Y_{2}^{\prime} \oplus X_{3}, \\
S_{21} \mathfrak{g} & =2 \mathfrak{g} \oplus X_{2} \oplus Y_{2} \oplus Y_{2}^{\prime} \oplus A \oplus C \oplus C^{\prime}
\end{aligned}
$$

where the (normalized) Casimir operator acts on $X_{2}, X_{3}, A, Y_{2}, Y_{2}^{\prime}, Y_{3}, Y_{3}^{\prime}, C, C^{\prime}$ by multiplication by $2,3,8 / 3,(2 \beta+5 \gamma) / 3 \gamma,(2 \alpha+5 \gamma) / 3 \gamma, 2(\beta+\gamma) / \gamma$, $2(\alpha+\gamma) / \gamma,(2 \beta+5 \gamma) / 2 \gamma,(2 \alpha+5 \gamma) / 2 \gamma$ respectively. Again, we are able to account for nearly all the factors and Casimir functions appearing using diagram induction.

\subsubsection{Dimension Formulas}

For each exceptional Lie algebra $\mathfrak{s l}_{2}, \mathfrak{s l}_{3}, \mathfrak{s o}_{8} \mathfrak{g}_{2}, \mathfrak{f}_{4}, \mathfrak{e}_{6}, \mathfrak{e}_{7}, \mathfrak{e}_{8}$, let $\lambda=$ $-3,-2,-1,-3 / 2,-2 / 3,-1 / 2,-1 / 3,-1 / 5$ respectively. $\lambda$ is a linear function 
of the length of the longest root (with the Casimir normalized to act as the identity on $\mathfrak{g})$. In Vogel's parametrization this gives $(\alpha, \beta, \gamma)=(\lambda, 1-\lambda, 2)$.

From the decomposition formulas stated above and the knowledge of the Casimir eigenvalue on each irreducible component, it is easy (at least with a computer) to calculate the dimensions of these components as rational functions of $\lambda$, if one knows the dimension of $\mathfrak{g}$ itself in terms of $\lambda$. For example,

$$
\begin{gathered}
\operatorname{dimg}=-2 \frac{(\lambda+5)(\lambda-6)}{\lambda(\lambda-1)}, \\
\operatorname{dim} X_{2}=5 \frac{(\lambda+3)(\lambda+5)(\lambda-4)(\lambda-6)}{\lambda^{2}(\lambda-1)^{2}}, \\
\operatorname{dim} Y_{2}=-90 \frac{(\lambda+5)(\lambda-4)}{\lambda^{2}(\lambda-1)(2 \lambda-1)}, \\
\operatorname{dim} Y_{3}=-10 \frac{(\lambda+5)(5 \lambda-6)(\lambda-4)(\lambda-5)(\lambda-6)}{\lambda^{3}(\lambda-1)^{2}(2 \lambda-1)(3 \lambda-1)} .
\end{gathered}
$$

A miracle, Deligne says, is that the numerators and denominators of these rational functions all factor out into products of linear forms with simple integers coefficients! Note that this miracle does not occur for the Vogel dimension formulas starting with degree three.

In addition, there is a striking duality property: the involution $\lambda \mapsto 1-\lambda$ takes the dimension formula for $Y_{2}, Y_{3}$ into those for $Y_{2}^{\prime}, Y_{3}^{\prime}$, while it leaves unchanged the dimension formulas for $\mathfrak{g}, X_{2}, X_{3}, A$.

One can attempt to continue the decompositions in degree five, but there for the first time there appear different representations with the same Casimir eigenvalue and Deligne's method cannot be used to compute dimensions. This suggests that if one wants to continue the formulas, it might be better to restrict to decompositions into Casimir eigenspaces rather than irreducible modules.

In the last part of [24], Deligne conjectured the existence of a category, supposed (just as the universal Lie algebra defined by Vogel, but with much stronger properties) to map to the categories of modules over each exceptional Lie algebra. The properties of this hypothetic category would then explain at least some of the phenomena discovered by Vogel and Deligne for the exceptional series. At the moment, it seems no progress has been made on this conjecture.

\subsubsection{Deligne Dimension Formulas Via Triality}

Deligne's conjecture is elegant while his proof is brute force computer based. In [62] we reprove and extend his formulas using methods whose level of elegance is somewhere between the conjecture and proof of Deligne. The starting point was our observation that the parameter $\lambda$ could be written as $\lambda=-2 /(a+2)$, with $a=-2 / 3,1,2,4,8$. (This implies that $(\alpha, \beta, \gamma)=(-2, a+4,2 a+4)$.) 
This indicated that the relation between exceptional Lie algebras and normed division algebras should be exploited. The idea was to use this relation to find a good description of the exceptional root systems, and then apply the Weyl dimension formula.

The Tits construction is not convenient to describe the exceptional root systems and we were led to rediscover the triality model. Recall that for the exceptional series,

$$
\mathfrak{g}(\mathbb{A}, \mathbb{O})=\mathfrak{t}(\mathbb{A}) \times \mathfrak{t}(\mathbb{O}) \oplus\left(\mathbb{A}_{1} \otimes \mathbb{O}_{1}\right) \oplus\left(\mathbb{A}_{2} \otimes \mathbb{O}_{2}\right) \oplus\left(\mathbb{A}_{3} \otimes \mathbb{O}_{3}\right),
$$

can be identified with the compact form of $\mathfrak{f}_{4}, \mathfrak{e}_{6}, \mathfrak{e}_{7}, \mathfrak{e}_{8}$ respectively for $\mathbb{A}=$ $\mathbb{R}, \mathbb{C}, \mathbb{H}, \mathbb{O}$. In what follows we complexify this construction, without changing notation. In particular $\mathfrak{t}(\mathbb{O})$ is now the complex orthogonal Lie algebra $\mathfrak{s o}_{8}(\mathbb{C})$. The point is that $\mathfrak{t}(\mathbb{A}) \times \mathfrak{t}(\mathbb{O})$ is now a maximal rank reductive subalgebra of $\mathfrak{g}(\mathbb{A}, \mathbb{O})$. In particular, if we choose Cartan subalgebras $\mathfrak{h}(\mathbb{A}) \subset \mathfrak{t}(\mathbb{A})$ and $\mathfrak{h}(\mathbb{O}) \subset \mathfrak{t}(\mathbb{O})$, we obtain a Cartan subalgebra $\mathfrak{h}(\mathbb{A}) \times \mathfrak{h}(\mathbb{O}) \subset \mathfrak{g}(\mathbb{A}, \mathbb{O})$. This provides a nice description of the root system of $\mathfrak{g}(\mathbb{A}, \mathbb{O})$. There are three kinds of roots:

- the heart of the root system is the set of roots of $\mathfrak{t}(\mathbb{O})=\mathfrak{s o}_{8}(\mathbb{C})$,

- the linear part is made of the roots of the form $\mu+\nu$, where $\mu$ is a weight of $\mathbb{O}_{i}$ and $\nu$ a weight of $\mathbb{A}_{i}$ for some $i$,

- the residue is the set of roots of $\mathfrak{t}(\mathbb{A})$.

When $\mathbb{A}=\underline{0}$, the root system is of type $D_{4}$. When $\mathbb{A}=\mathbb{R}$, the root system is of type $F_{4}$. There is no residue in this case. The heart is the set of long roots, and the linear part the set of short roots. Both are root systems of type $D_{4}$. When we change $\mathbb{A}$ into $\mathbb{C}, \mathbb{H}, \mathbb{O}$, the linear part expands, is size being each time multiplied by two, and its roots become long. The residue also increases, but in limited proportions.

Next we specify the positive roots inside the exceptional root systems. They are the roots on which some linear form takes positive values, and we choose a linear form that takes very large values on the set $\Delta_{+}$of positive roots of $\mathfrak{s o}_{8}(\mathbb{C})$. Then the positive roots in the linear part of the root system are the $\mu+\nu$ for $\mu$ belonging to an explicit set $\Sigma$ of weights. This implies that the half-sum of the positive roots can be expressed as $\rho=\rho_{\mathrm{t}(\mathbb{A})}+\rho_{\mathrm{t}(\mathbb{O})}+a \gamma_{\mathrm{t}(\mathbb{O})}$, where $\rho_{\mathfrak{t}(\mathbb{A})} \in \mathfrak{h}(\mathbb{A})^{*}$ denotes the half sum of the positive roots in $\mathfrak{t}(\mathbb{A})$ and $\gamma_{\mathfrak{t}(\mathbb{O})} \in \mathfrak{h}(\mathbb{O})^{*}$ is the sum of the weights belonging to $\Sigma$.

We are now close to being able to apply the Weyl dimension formula to $\mathfrak{g}(\mathbb{A}, \mathbb{O})$-modules. What remains to do is to describe the dominant integral weights in $\mathfrak{h}(\mathbb{A})^{*} \times \mathfrak{h}(\mathbb{O})^{*}$. At least we can describe the set $C(\mathbb{O}) \subset \mathfrak{h}(\mathbb{O})^{*}$ of weights that are dominant and integral for each $\mathfrak{g}(\mathbb{A}, \mathbb{O})$. Of course, such weights are be dominant and integral in $\mathfrak{s o}_{8}(\mathbb{C})$, but this is not sufficient.

Proposition 4.2. The set $C(\mathbb{O}) \subset \mathfrak{h}(\mathbb{O})^{*}$ is the simplicial cone of nonnegative integer linear combinations of the four following weights: 


$$
\begin{gathered}
\omega(\mathfrak{g})=1-2 \nearrow_{1}^{1}, \quad \omega\left(X_{2}\right)=2-3 \nearrow_{2}^{2}, \\
\omega\left(X_{3}\right)=3-4 \nearrow_{3}^{2}, \quad \omega\left(Y_{2}^{\prime}\right)=2-2 \nearrow_{1}^{1} .
\end{gathered}
$$

The lattice in $\mathfrak{t}(\mathbb{O})^{*}$ generated by $C(\mathbb{O})$ is not the weight lattice, but the root lattice of $\mathfrak{s o}_{8}(\mathbb{C})$.

These weights, that we expressed above in terms of simple roots, occur respectively, $\omega(\mathfrak{g})=\omega_{2}$ as the highest weight of $\mathfrak{g}, \omega\left(X_{2}\right)=\omega_{1}+\omega_{3}+\omega_{4}$ as the highest weight of $\Lambda^{2} \mathfrak{g}, \omega\left(X_{3}\right)=2 \omega_{1}+2 \omega_{3}$ as the highest weight of $\Lambda^{3} \mathfrak{g}$, and $\omega\left(Y_{2}^{\prime}\right)=2 \omega_{1}$ as the highest weight of $S^{2} \mathfrak{g}-\mathfrak{g}^{(2)}$.

We can now apply the Weyl dimension formula to weights in $C(\mathbb{O})$, considered for each choice of $\mathbb{A}$ as integral dominant weights of $\mathfrak{g}(\mathbb{A}, \mathbb{O})$. It is essential that the residue of the root system will not contribute. We obtain (see [62] for details):

Theorem 4.3. The dimension of the irreducible $\mathfrak{g}(\mathbb{A}, \mathbb{O})$-module of highest weight $\omega \in C(\mathbb{O})$ is given by the following formula:

$$
\begin{aligned}
\operatorname{dim} V_{\omega}= & \prod_{\alpha \in \Delta_{+} \cup \Sigma} \frac{\left(a \gamma_{\mathfrak{t}(\mathbb{O})}+\rho_{\mathfrak{t}(\mathbb{O})}+\omega, \alpha^{\vee}\right)}{\left(a \gamma_{\mathfrak{t}(\mathbb{O})}+\rho_{\mathfrak{t}(\mathbb{O})}, \alpha^{\vee}\right)} \\
& \times \prod_{\beta \in \Sigma} \frac{\left(\begin{array}{c}
\left(a \gamma_{\mathfrak{t}(\mathbb{O})}+\rho_{\mathfrak{t}(\mathbb{O})}+\omega, \beta^{\vee}\right)+\frac{a}{2}-1 \\
\left(\omega, \beta^{\vee}\right)
\end{array}\right.}{\left(\begin{array}{c}
\left(a \gamma_{\mathfrak{t}(\mathbb{O})}+\rho_{\mathfrak{t}(\mathbb{O})}+\omega, \beta^{\vee}\right)-\frac{a}{2} \\
\left(\omega, \beta^{\vee}\right)
\end{array}\right.}
\end{aligned}
$$

If $\omega=p \omega(\mathfrak{g})+q \omega\left(X_{2}\right)+r \omega\left(X_{3}\right)+s \omega\left(Y_{2}^{*}\right)$, this formula gives a rational function of $a$, whose numerator and denominator are products of $6 p+12 q+$ $16 r+10 s+24$ linear forms in $a$ with integer coefficients. Since $\lambda=-2 /(a+2)$, we obtain formulas of the type of those of Deligne, and an infinite family of such. For example, the $k$-th Cartan power $\mathfrak{g}(\mathbb{A}, \mathbb{O})^{(k)}$ is $Y_{k}$ in Deligne's notations, and we get

$$
\operatorname{dim} Y_{k}=\frac{(2 k-1) \lambda-6}{k ! \lambda^{k}(\lambda+6)} \prod_{j=1}^{k} \frac{((j-1) \lambda-4)((j-2) \lambda-5)((j-2) \lambda-6)}{(j \lambda-1)((j-1) \lambda-2)}
$$

Also we can understand why we can expect good dimension formulas for all irreducible components of $\mathfrak{g}(\mathbb{A}, \mathbb{O}) \otimes k$ only for small $k$. This is because when $k$ increases, we will unavoidably get components whose highest weight does not come only from $\mathfrak{s o}_{8}(\mathbb{C})$, but has a contribution from $\mathfrak{h}(\mathbb{A})^{*}$. Then the Weyl dimension formula does not give a well behaved expression.

While this takes some of the mystery out of the dimension formulas, the remarkable symmetry $\lambda \mapsto 1-\lambda$ noticed by Deligne, which in our parameter $a$ is $a \mapsto-4(a+3) /(a+4)$ remains beyond our understanding. 


\subsubsection{Numerology}

In the formula above for $\operatorname{dim} Y_{k}$, there are very few values of $\lambda$ for which $\operatorname{dim} Y_{k}$ is an integer for all $k$. We are currently, with $\mathrm{B}$. Westbury [65] investigating these extra numbers that produce integers and Lie algebras that go with them. At least in the case $a=6$, one is led to an algebra of dimension six, the sextonions, that leads to a nonreductive row of the magic square between the third and fourth. Like the odd symplectic groups of Proctor [73] and Gelfand and Zelevinsky [36], this series has certain behavior as if it were a series of reductive Lie algebras.

\subsection{Freudenthal Geometries}

The discovery of the Cayley plane $\mathbb{O P}^{2}$ and its automorphism group by Chevalley, Schafer and others led to a period of intense activity around the exceptional groups and their geometric interpretations in the 1950's involving Freudenthal, Tits and Rozenfeld.

Freudenthal and Rozenfeld defined explicit varieties whose automorphism groups (or groups related to the automorphism groups) were the exceptional groups. The starting point is the Jordan algebras $\mathcal{J}_{3}(\mathbb{A})$. The projective plane $\mathbb{A P}^{2}$ is the space of rank one idempotents of $\mathcal{J}_{3}(\mathbb{A})$. The subgroup of $G L\left(\mathcal{J}_{3}(\mathbb{A})\right)$ preserving the determinant of order three matrices (which is well defined even for $\mathbb{A}=\mathbb{O}$ ) then acts transitively on $\mathbb{A P}^{2}$. In particular, one recovers the transitive action of $E_{6}$ on $\mathbb{O P}^{2}$. The subgroup of $\operatorname{GL}\left(\mathcal{J}_{3}(\mathbb{A})\right)$ preserving the determinant and the quadratic form $(A, B)=\operatorname{trace}(A B)$ is the automorphism group of the Jordan algebra $\mathcal{J}_{3}(\mathbb{A})$. It acts irreducibly on $\mathcal{J}_{3}(\mathbb{A})_{0}$, the subspace of trace zero matrices. Tits and Freudenthal defined elliptic and projective geometries on $\mathbb{A P}^{2}$ with these groups of isometries. In the elliptic geometry, a point is the same as a line, and is defined by an element of $\mathbb{A P}_{0}^{2}$. In the projective geometry, a point is defined to be an element of $\mathbb{A P}^{2}$ and a line is determined by an element $[\alpha]$ of the dual projective plane (the rank one idempotents in the dual Jordan algebra corresponding to the dual vector space), namely $\left\{X \in \mathbb{A P}^{2} \mid \alpha(X)=0\right\}$.

A synthetic geometry Freudenthal terms symplectic may be associated to each of the groups in the third row. A point is an element $[X] \in \mathbb{P} \mathfrak{g}(\mathbb{A}, \mathbb{H})$, such that $\operatorname{ad}(X)^{2}=0$. This condition is equivalent to requiring that $[X]$ is in the adjoint variety $X_{\text {ad }} \subset \mathbb{P} \mathfrak{g}(\mathbb{A}, \mathbb{H})$. A plane is is determined by an element $[P] \in \mathbb{P} \mathcal{Z}_{3}(\mathbb{A}), P \times P=0$. (See Section 4.6 for the definition of the cross product.) This condition is equivalent to saying that $[P] \in G_{w}\left(\mathbb{A}^{3}, \mathbb{A}^{6}\right) \subset$ $\mathbb{P} \mathcal{Z}_{3}(\mathbb{A})$, the closed orbit. The corresponding plane is $\left\{[X] \in X_{\mathrm{ad}} \mid X P=0\right\}$. A line is an intersection of two planes with at least two points. It is also determined by a point $[\alpha] \in \mathbb{F}_{1}(X) \subset \mathbb{P} V_{2}$, where $V_{2}$ is the representation defined in Section 4.6 below and $\mathbb{F}_{1}\left(G_{w}\left(\mathbb{A}^{3}, \mathbb{A}^{6}\right)\right)$ is the closed orbit (which, as the notation suggests, parametrizes the lines on the closed orbit $G_{w}\left(\mathbb{A}^{3}, \mathbb{A}^{6}\right) \subset$ 
$\left.\mathbb{P} \mathcal{Z}_{3}(\mathbb{A})\right)$. One can then define incidence rules for these geometric elements which generalize the symplectic geometry in $\mathbb{P}^{5}$.

For the fourth line of Freudenthal's magic square Freudenthal defines a metasymplectic geometry. There are now four types of elements, points, lines, planes and symplecta, with incidence rules explained in detail by Freudenthal. As above, each geometric element is a point in the closed orbit of the projectivization of a $\mathfrak{g}(\mathbb{A}, \mathbb{O})$-module. Here is the table of types of elements:

\begin{tabular}{|c|c|c|}
\hline Geometric element & Variety & Dimension \\
\hline Point & $X_{\text {point }} \subset \mathbb{P} \mathfrak{g}_{Q}$ & $9 a+6$ \\
\hline Line & $X_{\text {line }} \subset \mathbb{P}_{3}$ & $11 a+9$ \\
\hline Plane & $X_{\text {plane }} \subset \mathbb{P g}_{2}$ & $9 a+11$ \\
\hline Symplecta & $X_{\text {symplecta }} \subset \mathbb{P} \mathfrak{g}(\mathbb{A}, \mathbb{O})$ & $6 a+9$ \\
\hline
\end{tabular}

We see the four generators of the cone $C(\mathbb{O})$ in Proposition 4.2 define the four types of elements of Freudenthal's metasymplectic geometry! The analogous results hold for the second and third rows of the magic chart, that is, Freudenthals elements are the closed orbits in the projectivizations of the modules whose highest weights define the fixed cones for our dimension formulas in [62].

\subsection{A Geometric Magic Square}

Consider the following square of complex homogeneous varieties, which we investigate in [61]:

\begin{tabular}{|c|c|c|c|c|c|}
\hline & $\mathbb{R}$ & $\mathbb{C}$ & $\mathbb{H}$ & (1) & \\
\hline $\mathbb{R}$ & $\overline{v_{2}\left(Q^{1}\right)}$ & $\mathbb{P}\left(T \mathbb{P}^{2}\right)$ & $G_{\omega}(2,6)$ & $\mathbb{O P}_{\mathbb{C}, 0}^{2}$ & section of Severi \\
\hline $\mathbb{C}$ & $v_{2}\left(\mathbb{P}^{2}\right)$ & $\mathbb{P}^{2} \times \mathbb{P}^{2}$ & $G(2,6)$ & $\mathbb{O P} \mathbb{P}_{\mathbb{C}}^{2}$ & Severi \\
\hline $\mathbb{H}$ & $G_{\omega}(3,6)$ & $G(3,6)$ & $\mathbb{S}_{12}$ & $E_{7} / P_{7}$ & Legendre \\
\hline $\mathbb{1}$ & $F_{4}^{a d}$ & $E_{6}^{a d}$ & $E_{7}^{a d}$ & $E_{8}^{a d}$ & adjoint \\
\hline
\end{tabular}

This geometric magic square is obtained as follows. One begins with the adjoint varieties for the exceptional groups: this is the fourth line of the square. Taking the varieties of lines through a point and applying Theorem 2.5, one obtains the third line. The second line is deduced from the third by the same process. Then take hyperplane sections to get the first line. We obtain projective varieties that are homogeneous under groups given by Freudenthal's magic square.

\subsection{The Subexceptional Series}

In this final section we consider the series corresponding to the third row of the extended Freudenthal chart: 


$$
\mathfrak{s l}_{2}, \quad \mathfrak{s l}_{2} \times \mathfrak{s l}_{2} \times \mathfrak{s l}_{2}, \quad \mathfrak{s p}_{6}, \quad \mathfrak{s l}_{6}, \quad \mathfrak{s o}_{12}, \quad \mathfrak{e}_{7}
$$

and view the series from our various perspectives.

\subsubsection{Relation To the Universal Lie Algebra}

This series also corresponds to a new line in Vogel's plane, namely $(\alpha, \beta, \gamma)=$ $(-2, a, a+4)$, where $a=-2 / 3,0,1,2,4,8$. Moreover, Vogel's formulas are valid for the semi-simple Lie algebra $\mathfrak{s l}_{2} \times \mathfrak{s l}_{2} \times \mathfrak{s l}_{2}$ despite the plane being defined only for absolutely simple objects. Unlike the three lines discovered by Vogel, this line is generic to order three among actual Lie algebras, in the sense that no Vogel space is zero except $X_{3}^{\prime \prime}$, which is zero for all actual Lie algebras. We have $\operatorname{dim} \mathfrak{g}(a)=3(2 a+3)(3 a+4) /(a+4)$.

\subsubsection{Freudenthalia}

For this series there are three preferred representations from Freudenthal's perspective, corresponding to the ambient spaces for points, lines, and planes in his incidence geometries. The points representation is just $\mathfrak{g}=\mathfrak{g}(\mathbb{A}, \mathbb{H})$ and has a nice model thanks to the triality construction. For a discussion of the line space, which we will denote $V_{2}=V_{2}(a)$ see [61]. We have $\operatorname{dim} V_{2}(a)=$ $9(a+1)(2 a+3)$. The most preferred representation is for the planes, which we denote $V=V(a)$ and has $\operatorname{dim} V(a)=6 a+8$. This space is the complexification of the algebra of Zorn matrices we encountered in Section 3.8:

$$
\mathcal{Z}_{3}(\mathbb{A})=\left\{\left(\begin{array}{cc}
a & X \\
Y & b
\end{array}\right) \mid a, b \in \mathbb{R}, X, Y \in \mathcal{J}_{3}(\mathbb{A})\right\} .
$$

It can be endowed with an algebra structure with multiplication

$$
\left(\begin{array}{cc}
a_{1} & X_{1} \\
Y_{1} & b_{1}
\end{array}\right)\left(\begin{array}{cc}
a_{2} & X_{2} \\
Y_{2} & b_{2}
\end{array}\right)=\left(\begin{array}{cc}
a_{1} a_{2}+\operatorname{trace}\left(X_{1} Y_{2}\right) & a_{1} X_{2}+b_{2} X_{1}+Y_{1} \times Y_{2} \\
a_{2} Y_{1}+b_{1} Y_{2}+X_{1} \times X_{2} & b_{1} b_{2}+\operatorname{trace}\left(X_{2} Y_{1}\right)
\end{array}\right) .
$$

Here the product $X \times Y$ is defined by the identity $\operatorname{tr}((X \times Y) Z)=\operatorname{det}(X, Y, Z)$, where det is the polarization of the determinant. The algebra is in fact a structurable algebra (see Section 2.5), with $V(a)=\mathcal{Z}_{3}(\mathbb{A})=\left\langle Y_{x}\right\rangle \subset$ $T_{x} X_{\text {adjoint }}(\mathbb{A}, \mathbb{O})$ where $X_{\text {adjoint }}(\mathbb{A}, \mathbb{O})$ are the exceptional adjoint varieties. In fact, Zorn matrices give all simple structurable algebras of skew-symmetric rank equal to one, and one can show directly that the derivation algebra of $\mathcal{Z}_{3}(\mathbb{A})$ is $\mathfrak{g}(\mathbb{A}, \mathbb{H})$.

Using this construction one can consider the automorphism group of $\mathcal{Z}_{3}(\mathbb{A})$ in addition to its derivation algebra, and this can be done on any field (of characteristic not equal to 2 or 3 ). Using this, Garibaldi constructs forms of $E_{7}$ over an arbitrary field [33].

The closed orbit $X_{\text {planes }} \subset \mathbb{P} \mathcal{Z}_{3}(\mathbb{A})$ is a natural compactification of the Jordan algebra $\mathcal{J}_{3}(\mathbb{A})$, and its embedding inside $\mathbb{P} \mathcal{Z}_{3}(\mathbb{A})$ is given by the translates 
of the determinant on $\mathcal{J}_{3}(\mathbb{A})$. This is the conformal compactification considered by Faraut and Gindikin in [30]. In [61] we propose a geometric interpretation of $X_{\text {planes }}$ as the Grassmannian of $\mathbb{O}^{3}$ 's in $\mathbb{O}^{6}$ isotropic for a Hermitian symplectic form and use the notation $G_{w}\left(\mathbb{O}^{3}, \mathbb{O}^{6}\right)$.

\subsubsection{Decomposition Formulas}

The plethysms of $V$ are extraodinarily well behaved in series. For example, here is a decomposition of $S^{k} V$ into irreducible components for each $k$.

$$
\bigoplus_{k \geq 0} t^{k} S^{k} V=(1-t V)^{-1}\left(1-t^{2} \mathfrak{g}\right)^{-1}\left(1-t^{3} V\right)^{-1}\left(1-t^{4}\right)^{-1}\left(1-t^{4} V_{2}\right)^{-1}
$$

The right hand side in the formula is to be expanded out in geometric series and multiplication of representations is taken in terms of Cartan products $V_{\lambda} V_{\mu}=V_{\lambda+\mu}$. The case of $\mathfrak{s l}_{2} \times \mathfrak{s l}_{2} \times \mathfrak{s l}_{2}$ is somewhat special, since the formula above holds true only if the natural action of $\mathfrak{S}_{3}$ is taken into account. See [63].

Each of the factors in the rational function above can be accounted for in terms of diagram induction. In particular, $\mathfrak{g}=V_{Q}$, the ambient space for the variety parametrizing the $G$-homogeneous quadrics on $X_{\text {planes }}$ and $V_{2}=\left\langle\mathbb{F}_{1}\left(X_{\text {planes }}\right)\right\rangle$ is the ambient space for the variety parametrizing the lines on $X_{\text {planes. }}$.

\subsubsection{Dimension Formulas}

Our method for applying the Weyl dimension formula in series also works for the three distinguished representations. For example, we have

$$
\operatorname{dim} V^{(k)}=\frac{2 a+2 k+2}{a+1} \frac{\left(\begin{array}{c}
k+2 a+1 \\
2 a+1
\end{array}\right)\left(\begin{array}{c}
k+\frac{3 a}{2}+1 \\
\frac{3 a}{2}+1
\end{array}\right)}{\left(\begin{array}{c}
k+\frac{a}{2}+1 \\
\frac{a}{2}+1
\end{array}\right)}
$$

where the binomial coefficients are defined by $\left(\begin{array}{c}k+x \\ k\end{array}\right)=(1+x) \cdots(k+x) / k$ ! and thus are rational polynomials of degre $k$ in $x$.

Note that since $V^{(k)}$ is the complement of $I_{k}\left(X_{\text {planes }}\right)$ in $S^{k} V^{*}$, the above formula also gives the Hilbert functions of the varieties $X_{\text {planes }}$ in a uniform manner.

\subsubsection{Zakology}

The varieties $X_{\text {planes }} \subset \mathbb{P} V$ are important for the following geometric classification problem: Given a smooth variety $X \subset \mathbb{P} V$, one defines its dual variety $X^{*} \subset \mathbb{P} V^{*}$ to be the set of hyperplanes tangent to $X$. Usually the degree of $X^{*}$ is quite large with respect to $X$ and Zak has proposed the problem of classifying smooth varieties whose duals are of low degree [86]. 
Here the duals of $X_{\text {planes }} \subset \mathbb{P} V$ are of degree four. Moreover, they are the tangential varieties of the closed orbits in the dual projective space, i.e., we have $X_{\text {planes }}^{*} \simeq \tau\left(X_{\text {planes }}\right)$.

The first variety in the series is $X_{\text {planes }}(-2 / 3)=v_{3}\left(\mathbb{P}^{1}\right)$ and the equation of its dual is the classical discriminant of a cubic polynomial. Using $\mathbb{A}$-valued variables, we write the equations for the duals in a uniform fashion, see [61]. In particular, we characterize the other quartics by their restriction to the preferred subspace. The second variety in the series is $X=\operatorname{Seg}\left(\mathbb{P}^{1} \times \mathbb{P}^{1} \times \mathbb{P}^{1}\right)$ and its dual is Cayley's hyperdeterminant, see [35]. Our restriction result gives a new characterization of the hyperdeterminant.

\subsubsection{Orbits}

The orbit structure is also uniform.

Proposition 4.4. [61] For each of the varieties $X_{\text {planes }}=G_{w}\left(\mathbb{A}^{3}, \mathbb{A}^{6}\right) \subset \mathbb{P} V$ there are exactly four orbits, the closures of which are ordered by inclusion:

$$
G_{w}\left(\mathbb{A}^{3}, \mathbb{A}^{6}\right) \subset \sigma_{+}\left(G_{w}\left(\mathbb{A}^{3}, \mathbb{A}^{6}\right)\right) \subset \tau\left(G_{w}\left(\mathbb{A}^{3}, \mathbb{A}^{6}\right)\right) \subset \mathbb{P} V .
$$

The dimensions are respectively $3 a+3,5 a+3$ and $6 a+6$.

Needless to say, the singular orbit closures have uniform desingularizations by Kempf's method.

Once again, the triality model plays a unifying role for geometric and representation geometric phenomena which at first glance seem sporadic. Another striking example of this role, which is currently under investigation, concerns nilpotent orbits in exceptional Lie algebras and the associated unipotent characters of exceptional Chevalley groups [66].

\section{References}

[1] Alexander, J., Hirschowitz, A.: Polynomial interpolation in several variables. J. Algebraic Geom. 4(2) 201-222 (1995)

[2] Allison B.N.: A class of nonassociative algebras with involution containing the class of Jordan algebras. Math. Ann. 237(2) 133-156 (1978)

[3] Allison, B.N., Faulkner, J.R.: Nonassociative coefficient algebras for Steinberg unitary Lie algebras. J. Algebra 161(1) 1-19 (1993)

[4] Arnol'd, V.I.: Normal forms of functions near degenerate critical points, the Weyl groups of $A_{k}, D_{k}, E_{k}$ and Lagrangian singularities. Funk. Anal. 6 3-25 (1972).

[5] Baez, J.: The octonions. Bull. Amer. Math. Soc. (N.S.) 39(2) 145-205 (2002)

[6] Bar-Natan, D.: On the Vassiliev knot invariants. Topology 34(2) 423-472 (1995)

[7] Barton, C.H., Sudbery, A.: Magic squares of Lie algebras. Preprint arXiv:math.RA/0001083 
[8] Beauville, A.: Fano contact manifolds and nilpotent orbits. Comment. Math. Helv. 73(4) 566-583 (1998)

[9] Bourbaki, N.: Groupes et algèbres de Lie. (Hermann, Paris 1968)

[10] Cartan, E.: Le principe de dualité et la théorie des groupes simples et semisimples. Bull. Sc. math. 49 361-374 (1925)

[11] Cartan, E.: Sur les variétés de courbure constante d'un espace euclidien ou noneuclidien. Collected Works. III. (Gauthier-Villars, Paris 1955) pp. 321-432.

[12] Cartan, E.: Sur des familles remarquables d'hypersurfaces isoparamétriques dans les espaces sphériques. Math. Z. 45 335-367 (1939)

[13] Carlini, E., Chipalkatti, J.: On Waring's problem for several algebraic forms. Preprint arXiv:math.ag/0112110.

[14] Cohen, A., de Man, R.: Computational evidence for Deligne's conjecture regarding exceptional groups. C.R.A.S. 322 427-432 (1996)

[15] Chaput, P.E.: Severi varieties. Math. Z. 240(2) 451-459 (2002)

[16] Chaput, P.E.: Scorza varieties and Jordan algebras. Indagationes Math. 14 169-182 (2003)

[17] Clerc, J.L.: Special prehomogeneous vector spaces associated to $F_{4}, E_{6}, E_{7}, E_{8}$ and simple Jordan algebras of rank 3. J. Algebra 264 (1) 98-128 (2003)

[18] Cohen, A., Cooperstein, B.: Lie incidence systems from projective varieties. Proc. Am. Math. Soc. 126 2095-2102 (1998)

[19] Cvitanović P.: Group theory for Feynman diagrams in non-Abelian gauge theories. Phys. Rev. D14 1536 (1976)

[20] Cvitanović P.: Negative dimensions and $E_{7}$ symmetry. Nucl. Phys. B188 373 (1981)

[21] Cvitanović P.: Group Theory. Available at www.nbi.dk/GroupTheory/, a monograph in preparation (1984-2002).

[22] Cohen, A.M., van Leeuwen, M.A., Lisser, B.: LiE, A Package for Lie Group Computations. (CAN, Amsterdam 1992)

[23] Dadok, J., Harvey, R.: A triality unification of Lie algebras. Preprint.

[24] Deligne, P.: La série exceptionnelle des groupes de Lie. C.R.A.S. 322 321-326 (1996)

[25] Deligne, P., de Man, R.: The exceptional series of Lie groups. C.R.A.S. 323 577-582 (1996).

[26] De Concini, C., Weyman, J.: A formula with nonnegative terms for the degree of the dual variaety of homogeneous space. Proc. AMS 125:1 1-8 (1997)

[27] Dixmier, J.: Algèbres enveloppantes. (Gauthier-Villars, Paris 1974)

[28] Dynkin, E.B.: Maximal subgroups of the classical groups. Amer. Math. Soc. Trans. Series 6 245-378 (1957)

[29] Ein, L., Shepherd-Barron, N.: Some special Cremona transformations. Amer. J. Math. 111(5) 783-800 (1989).

[30] Faraut, J., Gindikin, S.: Pseudo-Hermitian symmetric spaces of tube type. In: Gindikin, S. (ed.): Topics in Geometry, In Memory of Joseph D'Atri. (Birkhäuser, Basel 1996), pp. 123-154

[31] Freudenthal, H.: Oktaven, Ausnahmegruppen und Oktavengeometrie (notes from 1951). Geom. Dedicata 19 7-63 (1985)

[32] Freudenthal, H.: Lie groups in the foundations of geometry. Advances in Math. 1 145-190 (1964)

[33] Garibaldi, R.S.: Structurable algebras and groups of type $E_{6}$ and $E_{7}$. J. Algebra 236 651-691 (2001) 
[34] Gray, A., Wolf, J.: Homogeneous spaces defined by Lie group automorphisms. J. Diff. Geometry 2 77-114 and 115-159 (1968)

[35] Gelfand, I.M., Kapranov, M.M., Zelevinsky, A.V.: Discriminants, Resultants, and Multidimensional Determinants. Mathematics: Theory and Applications. (Birkhäuser, Basel 1994)

[36] Gelfand, I.M., Zelevinsky, A.V.: Representation models for classical groups and their higher symmetries. The mathematical heritage of Elie Cartan (Lyon, 1984). Numéro Hors Série (Astérisque 1985) pp. 117-128

[37] Griffiths, P.A., Harris, J.: Algebraic geometry and local differential geometry. Ann. scient. Ec. Norm. Sup. 12 355-432 (1979)

[38] Gross, B., Wallach, R.: On quaternionic discrete series representations and their continuations. J. reine angew. Math. 481 73-123 (196)

[39] Harvey, F.R.: Spinors and Calibrations. Perspectives in Math. vol. 9 (Academic Press, 1990)

[40] Hawkins: Emergence of the Theory of Lie Groups. An Essay in the History of Mathematics 1869-1926, Sources and Studies in the History of Mathematics and Physical Sciences. (Springer, New York 2000)

[41] Holweck, F.: Singularities of hyperplane sections of homogeneous varieties. In preparation

[42] Hong, J.: Fano manifolds with geometric structures modeled after homogeneous contact manifolds. Internat. J. Math. 11(9) 1203-1230 (2000)

[43] Howe, R.: Transcending classical invariant theory. J. Am. Math. Soc. 2 535-552 (1989)

[44] Hwang, J.-M., Mok, N.: Varieties of minimal rational tangents on uniruled projective manifolds. In: Schneider, M., Siu, Y.T. (eds): Several Complex Variables (Berkeley, CA, 1995-1996), 351-389, Math. Sci. Res. Inst. Publ. 37, Cambridge University Press 1999

[45] Hwang, J.-M., Mok, N.: Uniruled projective manifolds with irreducible reductive $G$-structures. J. reine Angew. Math. 490 55-64 (1997)

[46] Hwang, J.-M., Mok, N.: Rigidity of irreducible Hermitian symmetric spaces of the compact type under Kähler deformation. Invent. Math. 131(2) 393-418 (1998)

[47] Iarrobino, A., Kanev, V.: Power Sums, Gorenstein Algebras and Determinantal Loci. LNM 1721 (Springer, Berlin, Heidelberg 1999)

[48] Kac, V.: Infinite Dimensional Lie Algebras. 3rd edn. (Cambridge University Press, Cambridge 1990)

[49] Kac, V.: Some remarks on nilpotent orbits. J. Algebra, 64(1) 190-213 (1980)

[50] Kantor, I.: Certain generalizations of Jordan algebras. (Russian) Trudy Sem. Vektor. Tenzor. Anal. 16 407-499 (1972)

[51] Kebekus, S., Peternell, Th., Sommese, A., Wisniewski, J.: Projective contact manifolds. Invent. Math. 142 1-15 (2000)

[52] Kebekus, S.: Lines on contact manifolds. J. reine Angew. Math. 539 167-177 (2001)

[53] Kempf, G.: On the collapsing of homogeneous bundles. Invent. Math. 37 229239 (1976)

[54] Knop, F.: Ein neuer Zusammenhang zwischen einfachen Gruppen und einfachen Singularitaten. Invent. Math. 90 579-604 (1987)

[55] Kostant, B.: The principle of triality and a distinguished unitary representation of $\mathrm{SO}(4,4)$. Differential geometrical methods in theoretical physics. Proc. 16th Int. Conf., Como/Italy 1987, NATO ASI Ser., Ser. C 250, 65-108 (1988) 
[56] Kostant, B.: A cubic Dirac operator and the emergence of Euler number multiplets of representations for equal rank subgroups. Duke Math. J. 100(3) 447501 (1999)

[57] Landsberg, J.M.: On degenerate secant and tangential varieties and local differential geometry. Duke Math. J. 85 605-634 (1996)

[58] Landsberg, J.M.: On the infinitesimal rigidity of homogeneous varieties. Compositio Math. 118 189-201 (1999)

[59] Landsberg, J.M., Manivel, L.: On the projective geometry of homogeneous varieties. Commentari Math. Helv. 78 65-100 (2003)

[60] Landsberg, J.M., Manivel, L.: Classification of simple Lie algebras via projective geometry. Selecta Mathematica 8 137-159 (2002)

[61] Landsberg, J.M., Manivel, L.: The projective geometry of Freudenthal's magic square. J. Algebra 239 477-512 (2001)

[62] Landsberg, J.M., Manivel, L.: Triality, exceptional Lie algebras, and Deligne dimension formulas. Advances Math. 171 (1) 59-85 (2002)

[63] Landsberg, J.M., Manivel, L.: Series of Lie algebras. Preprint

[64] Landsberg, J.M., Manivel, L.: Legendrian and contact Fano varieties. In preparation

[65] Landsberg, J.M., Manivel, L., Westbury, B.: Sextonions and the dimension formulas of Cvitanović, Deligne and Vogel. In preparation

[66] Landsberg, J.M., Manivel, L., Westbury, B.: Series of nilpotent orbits. To appear in Experimental Math.

[67] Lascoux A.: Degree of the dual of a Grassmann variety. Comm. Algebra 9 $1215-1225(1981)$

[68] LeBrun, C.: Fano manifolds, contact structures, and quaternionic geometry. Internat. J. Math. 6 419-437 (1995)

[69] LeBrun, C., Salamon, S.: Strong rigidity of positive quaternion-Kahler manifolds. Invent. Math. 118 109-132 (1994)

[70] Loos O.: Symmetric spaces I and II. (Benjamin, 1969)

[71] Mukai, Sh.: Simple Lie algebra and Legendre variety. Nagoya Sūri Forum 3 1-12 (1996) (in Japanese). Revised translation into English: http://www.math. nagoya-u.ac.jp/ mukai/warwick15.pdf (1998) pp. 1-14

[72] Onishchik, A.L., Vinberg, E.B.: Lie Groups and Lie Algebras, III. Structure of Lie Groups and Lie Algebras. Encyclopaedia of Mathematical Sciences 41. (Springer, Berlin, Heidelberg 1994)

[73] Proctor, I.: Odd symplectic groups. Invent. Math. 92 307-332 (1988)

[74] Postnikov, M.: Groupes et algèbres de Lie (Editions Mir, Moscou 1985)

[75] Ranestad, K., Schreyer, F.-O.: Varieties of sums of powers. J. reine Angew. Math. 525 1455-1468 (2000)

[76] Rosenfeld, B.: Geometry of Lie Groups. Mathematics and its Applications, vol. 393 (Kluwer 1997)

[77] Rubenthaler, H.: Les paires duales dans les algèbres de Lie réductives. Astérisque 219 (1994)

[78] Tevelev, E.: Projectively Dual Varieties. Preprint math.AG/0112028

[79] Tits J.: Algèbres alternatives, algèbres de Jordan et algèbres de Lie exceptionnelles I. Construction. Indag. Math. 28 223-237 (1966)

[80] Tits, J.: Les groupes de Lie exceptionnels et leur interprétation géométrique. Bull. Soc. Math. Belg. 8 48-81 (1956)

[81] Tits, J.: Groupes semi-simples complexes et géométrie projective. Séminaire Bourbaki 7 (1954/1955), exposé 112, 11 pages. 
[82] Vogel, P.: Algebraic structures on modules of diagrams. Preprint 1996

[83] Vogel, P.: The universal Lie algebra. Preprint 1999

[84] Wolf, J.: Complex homogeneous contact manifolds and quaternionic symmetric spaces. Journal of Math. and Mech. 14 1033-1047 (1965)

[85] Zak, F.: Tangents and Secants of Algebraic Varieties. Translations of Math. Monographs 127, AMS 1993

[86] Zak, F.: Some properties of dual varieties and their applications in projective geometry. In: Algebraic Geometry, Proceedings, Chicago 1989, Springer LNM $1479,273-280$ 